\title{
Observation of $\Lambda$-Hypernuclei in the Reaction ${ }^{12} \mathrm{C}\left(\pi^{+}, \mathrm{K}^{+}\right)_{\Lambda}{ }^{12} \mathrm{C}$
}

\author{
Edward Casteel Milner*
}

\section{DISCLAIMER}

This report was prepared as an account of work sponsored by an agency of the United States Government. Neither the United States Government nor any agency thereof, nor any of their employves, makes any warranty, express or implied, or assumes any legal liability or responsibility for the accuracy, completeness, or usefulness of any information, apparatus, product, or process disclosed, or represents that its use would not infringe privately owned rights. Reference herein to any specific commercial product, process, or service by trade name, trademark, manufacturer, or otherwise does not necessarily constitute or imply its endorsement, recommendation, or favoring by the United States Government or any agency thereof. The views and opinions of authors expressed herein do not necessarily state or reflect those of the United States Government or any agency thercol. 
TABLE OF CONTENTS

Acknowledgments............................. viii

Abstract.............................. $x$

1. Introduction. ............................. 1

1.1 History............................. 2

2. Scientific motivation...................... 9

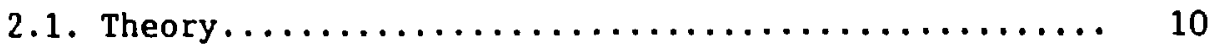

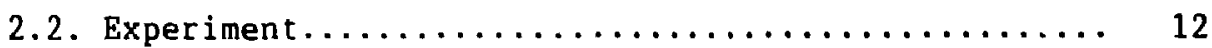

3. The experiment............................ 15

3.1. Selection of the pion momentum............. 16

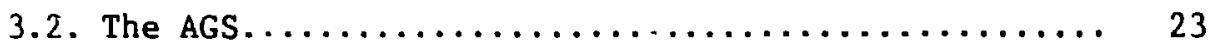

3.3. Pion beam and hypernuclear spectrometer systems.... 27

3.3.1. The mathematical model: TRANSPORT formalism.. 28

3.3.2. Experimental system overview.......... 36

3.3.3. LESB-I beam line................ 38

3.3.4. Pion spectrometer-Sp1............. 42

3.3.5. Kaon spectrometer- Sp2 ............ 45

3.3.6. Momentum calibration of $\mathrm{Spl}$ and $\mathrm{Sp} 2 \ldots \ldots \ldots 52$

3.4. Detectors........................... 60

3.4.1. Trigger counters................. 61

3.4.1.1. Time-of-flight scintillators....... 62

3.4.2. Position measuring detectors........... 63

3.4.2.1. Scintillator hodoscope.......... 63

3.4.2.2. High-rate drift chambers......... 64

3.4.2.3. Multi-wire proportional chambers.... 69 
3.5. Data acquisition..................... 71

3.5.1. Hardware....................... 71

3.5.1.1. Trigger electronics........... 72

3.5.1.2. CAMAC, MBD, and PDP-11/40....... 73

3.5.2. Software....................... 80

3.5.2.1. The analyzer................ 80

3.5.2.2. Data word testing............. 83

3.6. The scattering target................... 84

4. Experimental results........................ 89

4.1. Hypernuclear spectra................... 89

4.1.1. Energy resolution................. 95

4.2. Error analysis...................... 98

4.3. Differential cross sections................. 109

5. Theoretical calculation of the cross sections.......... 113

5.1. Shell model configurations............... 113

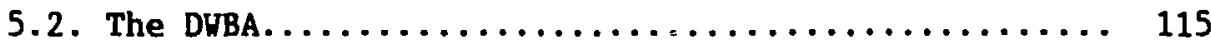

5.3. Fermi momentum averaging.................. 119

6. Conclusion............................... 121

6.1. Comparison with $\left(\mathrm{K}^{-}, \pi^{-}\right)$reaction results........ 121

6.2. Future prospects........................ 124

6.3. Summary........................... 126

Appendix $3.3 .3 . a \ldots \ldots \ldots \ldots \ldots \ldots \ldots \ldots \ldots \ldots \ldots \ldots \ldots \ldots \ldots$

Appendix $3.3 .4 . a \ldots \ldots \ldots \ldots \ldots \ldots \ldots \ldots \ldots \ldots \ldots \ldots \ldots \ldots \ldots$

Appendix $3.3 .4 . b \ldots \ldots \ldots \ldots \ldots \ldots \ldots \ldots \ldots \ldots \ldots \ldots \ldots \ldots \ldots$

Appendix $3.3 .5 . a \ldots \ldots \ldots \ldots \ldots \ldots \ldots \ldots \ldots \ldots \ldots \ldots \ldots \ldots \ldots$

Appendix $3.5 .2 .2 . a \ldots \ldots \ldots \ldots \ldots \ldots \ldots \ldots \ldots \ldots \ldots \ldots \ldots . \ldots \ldots$ 
Appendix $4.1 . a \ldots \ldots \ldots \ldots \ldots \ldots \ldots \ldots \ldots \ldots \ldots \ldots \ldots \ldots \ldots \ldots \ldots \ldots$

Appendix $5.2 . a \ldots \ldots \ldots \ldots \ldots \ldots \ldots \ldots \ldots \ldots \ldots \ldots \ldots \ldots \ldots \ldots \ldots$

References................................. 149 


\section{ACKNOWLEDGMENTS}

The observation of the $\left(\pi^{+}, \mathrm{K}^{+}\right)$hypernuclear reaction was a painstaking process, so the first thanks go to technical and scientific members of the experimental collaboration. In particular, I would like to express my deep gratitude to the following people for their hard work in this endeavor. Arch Thiessen proposed the experiment, and skillfully guided it to its completion. Phil pile was the force behind developing and maintainisg the often unruly high-rate drift chambers. Jim Amann and Tom Kozlowski developed and installed the $Q$ system for data acquisition -- and Jim worked on making the experiment a success with great stamina. To J. C. Peng I owe a deep debt of gratitude for performing some of the cross section calculations. Dick Silbar should be applauded for his fine work on the experiment, and showing some of the experimental physicists an organized approach to our work. Also Marti Barlett, Tarlochan Bhatia, John McGill, Hans Plendl, and Bob Stearns gave willingly of their time and considerable talents towards the success of these measurements. Special thanks are due to the AGS staff for keeping a rebellious accelerator running dependably. 
The talented efforts of Vito Manzella, Al Minn, Bill Espensen, Eddie Meier, and Jackie Moonie made the experiment possible, and enjoyable.

Thanks are due to Gerry Hoffmann for encouraging my envolvement in hypernuclear physics, and providing guidance and support throughout my graduate career. I also appreciate the support of The Robert A. Welch Foundation, Brookhaven National Laboratory, and Los Alamos National Laboratory during my research.

Bob Chrien was a source of experienced judgement and keen insight for me during this research, and should be credited for much of the approach taken to the analysis of this work. Cf course, any mistakes present herein are solely my responsibility. 
OBSERVATION OF $\Lambda$-HYPERNUCLEI IN THE REACTION ${ }^{12} \mathrm{C}\left(\pi^{+}, \mathrm{K}^{+}\right) \Lambda^{12} \mathrm{C}$

by

Edward Casteel Milner

ABSTRACT

The observation of $\Lambda$-hypernuclear levels in $\Lambda^{12} \mathrm{C}$ by associated production through the $\left(\pi^{+}, \mathrm{K}^{+}\right)$reaction is reported. Spectrometers used in the measurements are discussed. The $\Lambda^{12} \mathrm{C}$ excitation energy spectra were recorded at laboratory scattering angles of $5.6^{\circ}, 10.3^{\circ}$, and $15.2^{\circ}$. The spectra show two major peaks -- one attributed to the ground state, and one about $11 \mathrm{MeV}$ higher in excitation. The peak near $11 \mathrm{MeV}$ excitation energy is believed to be almost entirely composed of a multiplet of three $\mathrm{J}^{\pi}=2^{+}$states. Relativistic DWBA calculations imply support for the expectation that higher spin states are preferentially populated in the $\left(\pi^{+}, \mathrm{K}^{+}\right)$reaction, compared to the $\left(\mathrm{K}^{-}, \pi^{-}\right)$reaction in which lower spin states are excited. 
CHAPTER ONE

1. Introduction

Understanding the strong force, from fundamental principles, has proven to be elusive throughout the history of nuclear physics. The role of mesons as nuclear force propagators at long-range was established soon after their discovely. Meson-exchange theories have successfully explained a variety of nuclear phenomena, but fail the important test of describing the strong force at short-range. With the development of the quark model, quark-gluon interactions between baryons have become viewed as more fundamental than meson-exchange, particularly at short-range. If a quark theory arises which can explain all aspects of the NN interaction, it will provide the foundation for a comprehensive theory of the nucleus. Such a theory, being more fundamental, would naturally encompass meson-exchange processes, as well. In this context, it is appealing to consider experiments designed to reveal the possible contributions of quark-gluon interactions to nuclear phenomena.

A nucleus containing one or more hyperons is a generalized form of nuclear matter -- a hypernucleus -- composed of baryons made of $u, d$, and $s$ quarks. This constitutes a unique laboratory for exploring alterations to familiar nuclear phenomena arising from the 
presence of the distinguishable strange quark flavor. A fundamental quark interaction description of hypernuclear phenomena would be necessarily more general than one for the natural nucleus. A flavor independent, or "tasteless" theory, developed with insight gained from the study of hypernuclei, would describe regular nuclei by default. In this way, hypernuclear physics exposes new degrees of freedom of the nucleus, making opportunities for enhancing the information available for the eventual solution of the nuclear interaction problem.

\subsection{History}

In a 1966 paper, Feshbach and Kerman [Fe 66] pointed out the important "recoilless" feature of the $A\left(K^{-}, \pi^{-}\right) \Lambda^{A}$ reaction: for a $K^{-}$ momentum of about $550 \mathrm{MeV} / \mathrm{c}$, the momentum transfer is zero, and the $\Lambda$ is produced at rest. They speculated that the reaction form factor would have its maximum at this momentum, increasing the likelihood of trapping the $\Lambda$ in a bound state. These observations encouraged the development of experimental facilities designed to study the spectroscopy of in-flight production of hypernulcei. Previously, hypernuclei could be studied only in stopped-kaon reactions in emulsions $[\mathrm{Pn} 72]$. The emulsion work yielded the important $\wedge$ ground state binding energies for $s$ and $p$ shell nuclei. The types of information it is possible to gather with the emulsion 
technique are limited because: 1) all the fragments of hypernuclear decay must be uniquely identified in order to calculate the missing mass, and ; 2) only the ground state is usually observed, since excited hypernuclear states decay electromagnetically much faster than the hyperon decays.

Since there were many limitations in the stopped-kaon emulsion technique, and it was likely hypernuclei could be produced at a large rate by in-flight reactions, hypernuclear spectrometers were developed, besinning in 1970, at CERN and BNL utilizing the new intermediate-energy kaon beams. The first two counter experiments were at the CERN $\mathrm{PS}$, with the $\left(\mathrm{K}^{-}, \pi^{-}\right)$reaction at $\theta_{\mathrm{L}}=0^{\circ}$, collecting hypernuclear spectra in $\Lambda^{9} \mathrm{Be}, \Lambda^{12} \mathrm{C}, \Lambda^{16} 0[\mathrm{Br} 75]$, and $\Lambda^{160}, \Lambda^{27} \mathrm{Al}$ [Bo 75], at $\mathrm{P}_{\mathrm{K}}=900 \mathrm{MeV} / \mathrm{c}$ and $\mathrm{P}_{\mathrm{K}}=390 \mathrm{MeV} / \mathrm{c}$, respectively. Neither experiment was performed at zero momentum transfer, but as figure 1.1.a shows, the momentum transfer is relatively small over a broad range of incident kaon momenta. These spectra emphasized the formation of "substitutional" states, in which the $\Lambda$ assumes the quantum numbers of the neutron it replaces. Several spectra are shown in figure 1.1.b, and are typical of the resolution obtained in the early experiments. Two fixed-angle $\left(0^{\circ}\right)$ spectrometers, with a scattering target between them were used to measure the incoming and exiting particle momenta. Ground states were not observed, since for a hypernucleus the $\wedge$ is in the $1 s_{1 / 2}$ orbit, which is not generally a substitutional state. Excitation 


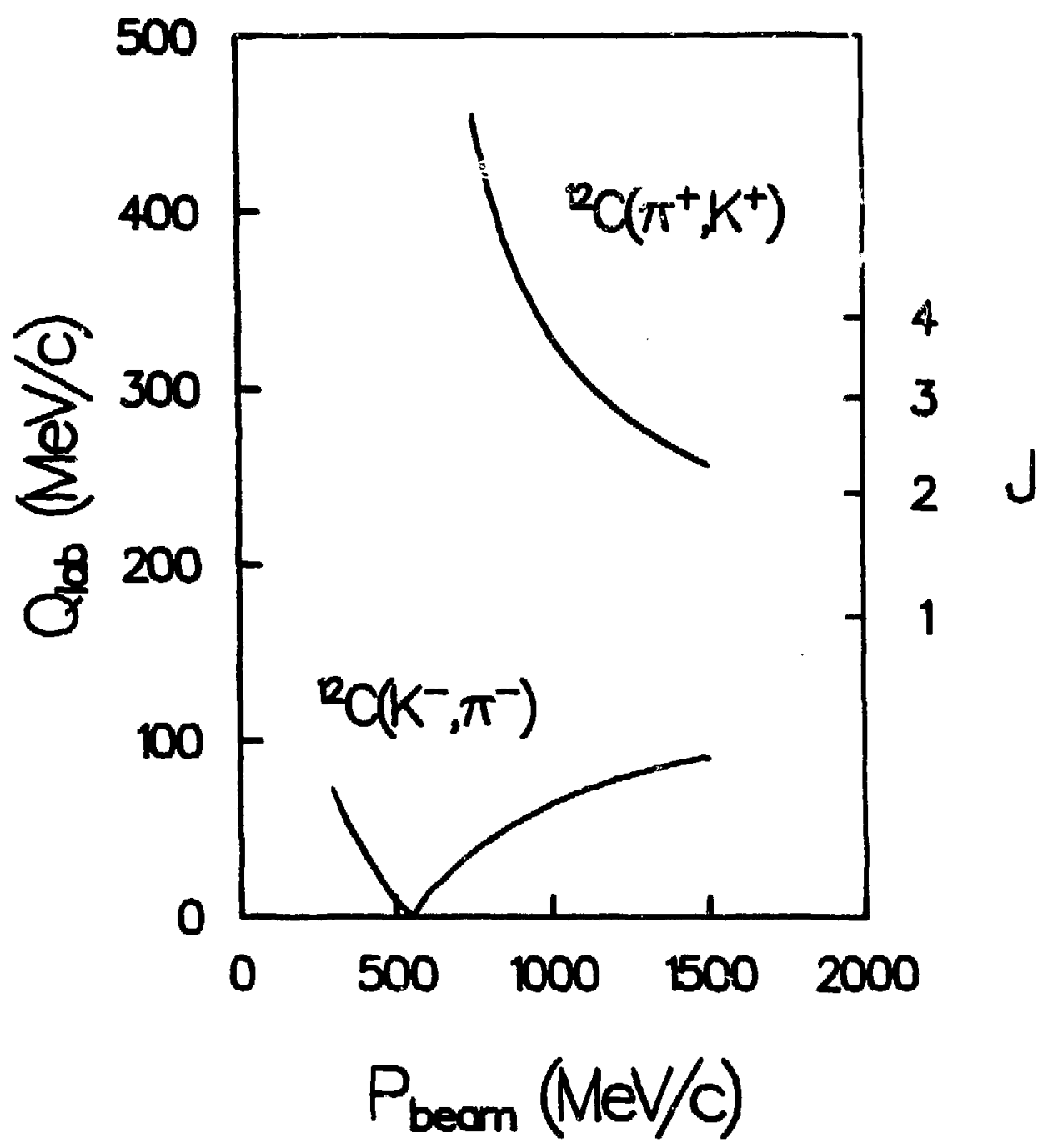

Figure 1.1.a. Momentum transfer $q$ (vertical scale, left-side), and optimum angular momentum $J$ for $\dot{\lambda}$-hypernuclear production as a function of incident kaon momentum in the $\left(\mathrm{K}^{-}, \pi^{-}\right)$reaction, and pion momentum in the $\left(\pi^{+}, \mathrm{K}^{+}\right)$reaction on ${ }^{12} \mathrm{C}$. The quantity $\mathrm{J}$ is calculated by maximizing the overlap of harmonic-oscillator wavefunctions, as described in the text. 

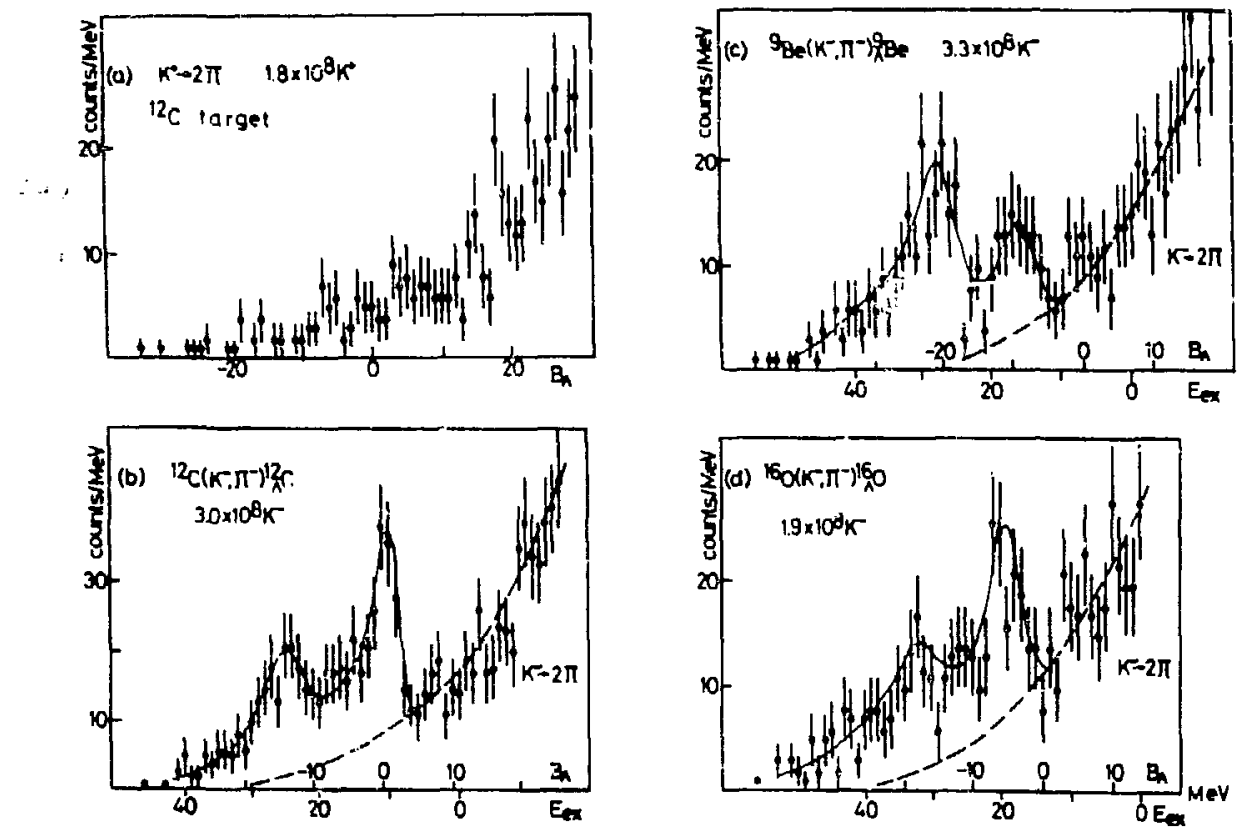

Figure 1.1.b. Spectra collected in the first hypernuclear magnetic spectrometer counter experiment at CERN. The $\left(K^{-}, \pi^{-}\right)$reaction was used, emphasizing substitutional states [Br 75]. 
energies for the observed states could be reliably assigned because missing mass was calculable knowing the binding energies from emulsion experiments. The states were interpreted as arising from neutron-hole $\Lambda$-particle couplings of simple structure, based on their energy spacings.

At the BNL AGS C2 beam line, the first hypernuclear cross section angular distributions were recorded [Ch 79]. These data, for ${ }^{12} \mathrm{C}\left(\mathrm{K}^{-}, \pi^{-}\right){ }_{\Lambda}^{12} \mathrm{C}$, revealed the strength of various $\mathrm{J}^{\pi}$ components of the spectral peaks. In the $11 \mathrm{MeV}$ excited peak multiplet (figure 1.1.c), three $2^{+}$and two $0^{+}$states arise from the $\left.\left({ }_{\Lambda} P_{3 / 2,1 / 2} \times{ }_{n} p_{3 / 2,1 / 2}\right)^{-1}\right)$ coupling. Their energy separation is small, because of the smallness of the $\Lambda$-hypernuclear spin-orbit splitting of $v_{\text {so }}-0.5 \mathrm{MeV}_{\text {? }}$ so the components are not separately resolvable with a magnetic spectrometer. However, it was shown that because the momentum transfer increases with angle, the marked shoulder in the angular distribution was produced by the $2^{+}$ contributions, while at smaller angles the cross section strength came mostly from $0^{+}$states.

The relationship between momentum transfer and the probability for forming certain angular momentum states, "momentum matching", is closeiy coinected to the choice of the reaction best suited to the physics of interest. The preferred angular momentum can be crudely estimated by maximizing the overlap of harmonic oscillator wavefunctions [Do 80 ], to give 


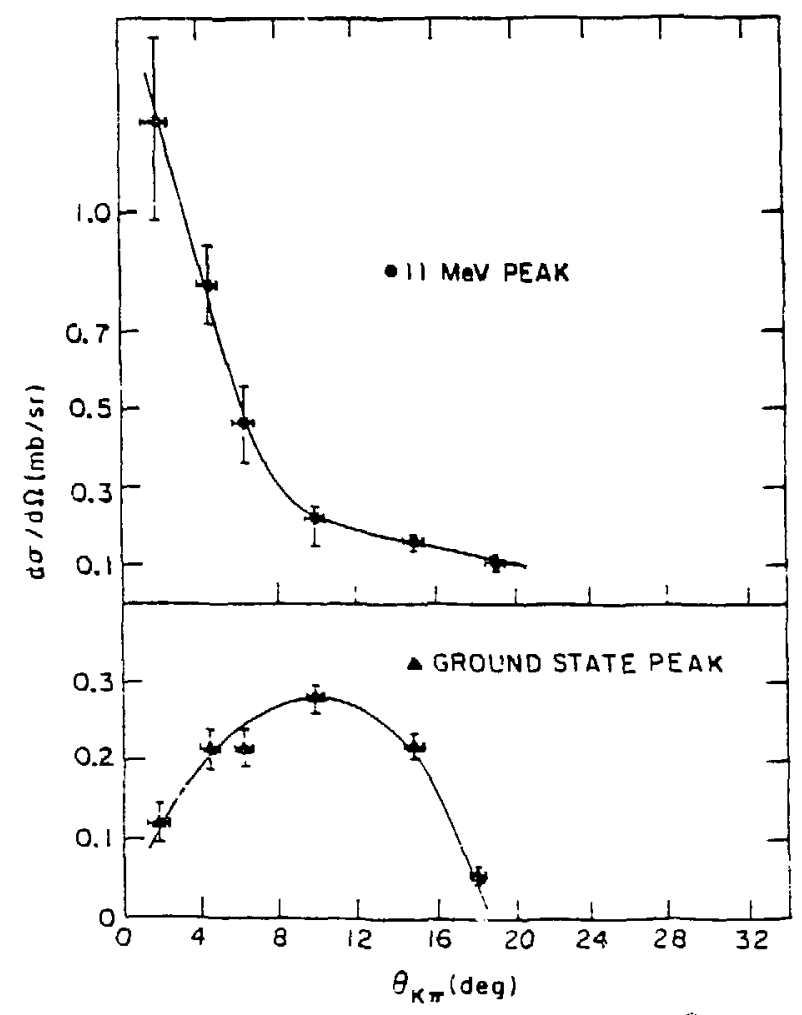

Figure 1.1.c. Cross section angular distributions measured in the $\left(\mathrm{K}^{-}, \pi^{-}\right)$reaction on ${ }^{12} \mathrm{C}$ by the BNL hypernuclear group. In the 11 Mt $V$ peak data, the small angle strength is attributed to $0^{+}$states, while at larger angles the shoulfer structure arises from $2^{+}$states [Ch 79]. 


$$
J=\frac{1}{2}(b q)^{2},
$$

eq. $1.1 \cdot a$

where $b=1.63$ fin for ${ }^{12} \mathrm{C}$ and $q$ is the momentum transfer. The angular momentum plotted against incident projectile momentum in figure 1.1.a was calculated using eq. 1.1.a. Evidently, the $\left(\mathrm{K}^{-}, \mathrm{\pi}^{-}\right)$reaction is constrained by the momentum matching condition to observation of states with $\Delta L=0$ and 1 , in contrast to the higher angular momentum preference in the $\left(\pi^{+}, \mathrm{K}^{+}\right)$reaction. For experiments on hypernuclei beyond the p-shell, such as $\Lambda^{28} \mathrm{Si}$ and $\Lambda^{40} \mathrm{Ca}$, other reactions with higher momentum transfer are better matched to the higher $J$ "stretched" siates which are expected to predominate. Stretched states are states of maximum angular momentum coupling of the particle and hole. In p-shell hypernuclei, the $\Lambda$ and hole can be separated by one shell, at most. But in heavier hypernuclei, the possible angular momentum is greater. The $\left(\pi^{+}, K^{+}\right)$reaction and the $\left(K^{-}, \pi^{-}\right)$reaction are therefore expected to provide complementary information. In fact, using the $\left(\pi^{+}, K^{+}\right)$ reaction may be the only practical way to study the predominately high spin states of heavy hypernuclei, including their ground state binding energies. 
CAAPTER TWO

2. Scientific motivation

often, science progresses by accumulating enough data on a particular poorly understood subject to allos the synthesis of a systematic description. An example of this process was the development of the periodic table by classification of the known elements by reaction characteristics. The recognition of patterns, spurred by the periodic scheme, led directly to the discovery of several elements, and precipitated the formulation of the electronic shell theory.

For hypernuclear physics, important binding energy and high spin data necessary to completing a comprehensive picture can only be obtained by reactions in which the momentum transfer is high. Emulsion experiments give $B_{\Lambda}$ only for $A \leq 16$, and little spectroscopy has been done on hypernuclei with $A \geq 16$. Since the study of hypernuclei has been confined to the $1 \mathrm{~s}$ and $2 \mathrm{p}$ shells by the low momentum transfer inherent to the $\left(\mathrm{K}^{-}, \pi^{-}\right)$reaction, it follows that valuable information about the systematic variation of $B_{\Lambda}$ and hypernuclear spin interactions beyond the p-shell can be obtained only in high $q$ reactions. Only with a full set of data on $B_{\mathcal{A}}$ can 
the $\Lambda$-nuclear interaction be reliably described from fundamental interactions .

Associated production of hypernuclei inherently transfers a large amount of momentum to the nucleus. The $r+p \rightarrow \Lambda+k^{+}$ reaction has been considered [Be 81 ], but the cross section is thought to be impracticaliy small, given present-day $\gamma$-fluxes and experimental techniques. The $\pi^{+}+n \rightarrow \Lambda+k^{+}$reaction, however, was predicted [Do 80] to have cross sections high enough, on the order of tens of $\mu \mathrm{b} / \mathrm{str}$, to be of practical use, especially given the high pion fluxes available.

\subsection{Theory}

There exist two theoretical motivations for studying $\Lambda$-hypernuclei with the $\left(\pi^{+}, \mathrm{K}^{+}\right)$reaction, both arising from the inherently large momentum transfer: 1) the possibility of testing descriptions of high-spin states, and ; 2) developing a systematic treatment of $\wedge$ binding energies over a wide mass range of nuclei.

The theoretical treatment of high-spin states potentially addresses many problems. The spin-orbit strength has been observed to be $v_{\text {so }} \sim 0.5 \mathrm{MeV}$, in $\wedge^{13} \mathrm{C}$, compared to $\sim 10 \mathrm{MeV}$ in the nucleon case. The smallness of the spin-orbit force was explained in the contexts of both meson-exchange and quark models [Pi 79]. In a stopped- $\mathrm{K}^{-}$experiment observing $\Sigma^{12} \mathrm{C} \quad$ [Ya 85], hovever, the 
spin-orbit splitting was deduced to be about $5.5 \mathrm{MeV}$. This result is not in agreement with meson-exchange (which predicts $v_{s_{0}}=0 \mathrm{MeV}$ ), but is in the range of a prediction made in a quark-cluster model [Mo 84]. The details of the spin-spin and spin-orbit interactions in high-spin states in $s-d$ shell $\Lambda$ - and $\Sigma$-hypernuclei, are open to study using the $\left(\pi^{+}, \mathrm{K}^{+}\right)$reaction.

The $\wedge$ binding potential, at long range, arises from the exchange of one kaon [ $\mathrm{Da} 58$ ]. This picture presents the opportunity to develop a flavor-independent description of meson-exchange forces, while working towards a tasteless quark-gluon model of the interaction, which may be required at short range. The $\Lambda$ in the nucleus is a distinguisiable baryon, so in the ground state it is always in the $1 s_{1 / 2}$ orbital, since it is not excluded by the Pauli principle. Therefore, $B_{\Lambda}$ is expected to be a more smoothly varying function of baryon number $A$ than in the nuclear case, where shell effects play an important role. A fit to the observad $B_{\Lambda}(3 \leq A \leq$ 16) values is obtainable with a simple square-well potential [Ro 70], giving

$$
\mathrm{B}_{\Lambda}=V_{0}-\frac{\pi^{2} \mathrm{R}^{2}}{2 \mathrm{M}_{\Lambda} \mathrm{ro}^{2}} A^{-2 / 3},
$$

where $V_{0}$ is the well depth, $r_{0}$ is the well radius, and $V_{0} \gg E_{\Lambda^{\circ}}$. With the adjustable parameters $r_{0}=1.1 \mathrm{fm}$ and $V_{0}=28 \mathrm{MeV}$, the 
observed $B_{\Lambda}$ values are reproducible. However, theories specifying meson-exchange mechanisms overbind the $\Lambda$. Speculative pictures in which only the $u$ and $d$ quarks are Pauli-blocked from the $1 s_{1 / 2}$ state, but the $s$ quark is not, would give smaller $B_{\Lambda}$ values. Implied in this view is a departure from the standard concept of total quark confinement, but other theories have also suggested in similar spirit expansions of the usual bag models. Clearly a larger body of data, including $B_{\Lambda}$ measurements for medium and heavy nuclei is necessary to the development of a correct theory of $\mu \mathrm{N}$ binding -perhaps contributing to an understanding of the NN interaction.

\subsection{Experiment}

The surprises in hypernuclear physics have come from experiment. Some examples are: the smallness of the $\Lambda$-hypernuclear spin-orbit potential, the larger $\Sigma$-hypernuclear spin-orbit pot itial, and the narrowness of $\Sigma$-hypernuclear states. Since the data primarily represent detailed studies of $p$ shell nuclei, new physics potentially exists in unexamined heavier hypernuclei. There are several aspects of the hypernuclear system which might be governed by interesting and unique effects. Requiring study are: 1) the spin-spin interaction, with high resolution $r$ detection in the $\left(\mathrm{K}^{-}, \pi^{-} \gamma\right)$ reaction ; 2$)$ ground state $\Lambda$ binding energies in medium and heavy nuclei; 3) high spin states; and 4) mirror hypernuclear 
systems. All of these experiments are accessible to the energy range of the present $500-1100 \mathrm{MeV} / \mathrm{c}$ BNL AGS C2 beam line. This is an important consideration, since the C2 line is presently the only facility in the world where in-flight reactions can be carried out (the advent of the proposed [Ch 85$] 2.0 \mathrm{GeV} / \mathrm{c}$ BNL separated beam line would of course multiply the experimenial possibilities). Note that studies of mirror hypernuclear systems (number four above) require a $\pi^{\circ}$ spectrometer to observe $\left(\mathrm{K}^{-}, \pi^{\circ}\right)$ in comparison with the $\left(\mathrm{K}^{-}, \pi^{-}\right)$data. Experiments one, two, and three can be performed with the existing spectrometer, while numbers two and three are feasible oniy using the $\left(\pi^{+}, \mathrm{K}^{+}\right)$reaction, with momentum of the incident beam at the high end of the $\mathrm{C} 2$ line capability.

For future facilities, the motivation to make hypernuclei with the $\left(\pi^{+}, K^{+}\right)$reaction might arise from practical considerations in addition to the physics interest. Typical $\pi: K$ secondary production ratios for $-30 \mathrm{GeV}$ proton-nucleus collisions are higher than 100:1. Al though the $\left(\pi^{+}, \mathrm{K}^{+}\right)$differential cross sections are expected to be in the $10 \mu \mathrm{b} / \mathrm{sr}$ range, compared to about $1 \mathrm{mb} / \mathrm{sr}$ in the $\left(\mathrm{K}^{-}, \pi^{-}\right)$ case, the pion beam fluxes compensate the disparity so that the event rates are expected to be similar. In the newly proposed higher momentum kaon beam line $(\sim 2 \mathrm{GeV} / \mathrm{c})$, a longer separator stage is required, as Enge has shown [En 84] that the optimum separator length is roughly twice the particle decay length. Therefore, if a kaon beam is used, the required separator can add cost and 
complexity to the experiment. However, if the $\left(\pi^{+}, \mathrm{K}^{+}\right)$rsaction is used, it may not be necessary to use a separator stage at all, an advantage with respect to the allow-ion of resources.

In summary, there sie several effects unique to hypernuclei, which are accessible only with the $\left(\pi^{+}, \mathrm{K}^{+}\right)$reaction, because of $i$ ts Sniferently high momentum transfer. Because of the expected preferential population of high spin states, the previously untested $\left(\pi^{+}, \mathrm{K}^{+}\right)$reaction might provide information complementary to the lower spin character of the $\left(\mathrm{K}^{-}, \pi^{-}\right)$reaction data. The following chapters describe the observation of spectral peaks in $\Lambda^{12} \mathrm{C}$ via the $\left(\mathrm{n}^{+}, \mathrm{K}^{+}\right)$reaction, which are interpreted as arising from $\left(\Lambda^{\mathrm{p}} \times{ }_{n} \mathrm{p}^{-1}\right)$ coupling to the maximum $\mathrm{J}=\mathrm{2}$. 
CHAPTER THREB

3. The experiment

Since the goal of this experiment was the first observation of the $\left(\pi^{+}, K^{+}\right)$hypernuclear reaction, several fundamental problems had to be explored before designing the apparatus. In this chapter the unique details of the design and execution of this experiment are presented. An explanation is made here of the determination of the incoming pion momentum maximizing the hypernuclear production. Once the beam requirements were set, the experimental facility was chosen to satisfy them. The Brookhaven National Laboratory hypernuclear spectrometers used in the study are discussed in this chapter, along with the instrumentation, while pointing out some of the novel design features of the high-rate drift chambers. Details of the data acquisition system are also presented. In the final section of the chapter, the live scattering target is discussed. 
3.1 Selection of the pion momentum

The event-rate for a nuclear reaction to a final state $\mathrm{J}^{\pi}$ can be estimated with the generalized expression

$$
I_{S}=I_{0} N_{0}\left[D \alpha N_{e f f} F\left[\left(\frac{d \sigma}{d \Omega}\right) \text { elem }\right] \Delta \Omega, \quad\right. \text { eq. 3.1.a }
$$

where $I_{S}$ is the scattered particle intensity, $I_{0}$ is the incident, particle intensity, $N_{0}$ is the number of scattering centers per unit volume times the target thickness, $D$ is the exiting particle decay survival rate, \& accounts for kinematic factors, $N_{\text {eff }}$ is the effective number of nucleons available for excitation to the state $\mathrm{J}^{\pi}, \mathrm{F}$ allows for averaging over the nucleon Fermi-distribution, $(d \sigma / d \Omega)$ elem is the elementary 2-body cross section, and $\Delta \Omega$ is the detector solid angle. Only $I_{0}, N_{0}$, and $\Delta \Omega$ do not depend on the incoming projectile momentum ( $\mathrm{P}_{\text {beam }}$ ) [Do 80]. Therefore, examination of the remaining factors allows estimation of the event-rate as a function of incident momentum. Then the projectile momentum maximizing the event-rate may be selected.

In the specific rase of the $\left(\mathrm{K}^{-}, \pi^{-}\right)$reaction, the choice of $P_{\text {beam }}$ is not critical, because eq. 3.1.a can be qualitatively shown to be a slowly varying function of momentum. Most experiments have been done in the $700-900 \mathrm{MeV} / \mathrm{C}$ range to avoid $\mathrm{K}^{-}$flux decay losses; losses which would be too large at the $-550 \mathrm{MeV} / \mathrm{c}$ "magic momentum" where zero momentum transfer occurs. Pion decay losses are not 
overwhelming in the $500-700 \mathrm{MeV} / \mathrm{c}$ range. The elementary cross section (figure 3.1.a) varies slowly over a wide (400-1000 MeV/c)

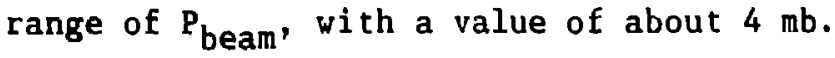

In contrast, the $\pi^{+}+n \rightarrow \Lambda+K^{+}$reaction (figure $3.1 . b$ ) is so sharply peaked at $P_{\pi}-1050 \mathrm{MeV} / \mathrm{c}$, one expects hypernuclear formation to be maximized at that momentum, the other factors in eq. 3.1.a notwithstanding. Determination of the rate-function in the $A\left(\pi^{+}, K^{+}\right) \Lambda^{A}$ reaction was initially discussed by Dover et al. [Do 80], and that treatment is closely followed here. However, previously undiscussed questions about the hypernuclear cross section peak position (witi respect to $P_{\pi}$ ) varying as a function of nuclear mass are raised in this section.

The most extensively studied $\pi \mathrm{H} \rightarrow \Lambda \mathrm{K}$ reaction is $\pi^{-}+p \rightarrow \Lambda+k^{0}$. Generally, 2-body reaction studies detect neutral products at zero degrees, igncring other channels by deflecting charged products away from detectors with a dipole magnet. But because of charge independence, the reaction of interest, $\pi^{+}+n \rightarrow \Lambda+K^{+}$, is equivalent. The structural behavior of the cross section is typical of a specific 2-body shannel; the peak in the cross section occurs near threshold $(0.89 \mathrm{GeV} / \mathrm{c})$, because at higher momenta, many other channels open. Comparatively, in the $\mathrm{K}^{-}+\mathrm{n} \rightarrow \Lambda+\pi^{-}$strangeness exchanging reaction, additional channels lie much higher, and are more widely spaced, so the cross section is not sharply peaked. 
REACTION $330.0 \quad K-F=L P I 0$

THAESHOLO $\quad: 3$

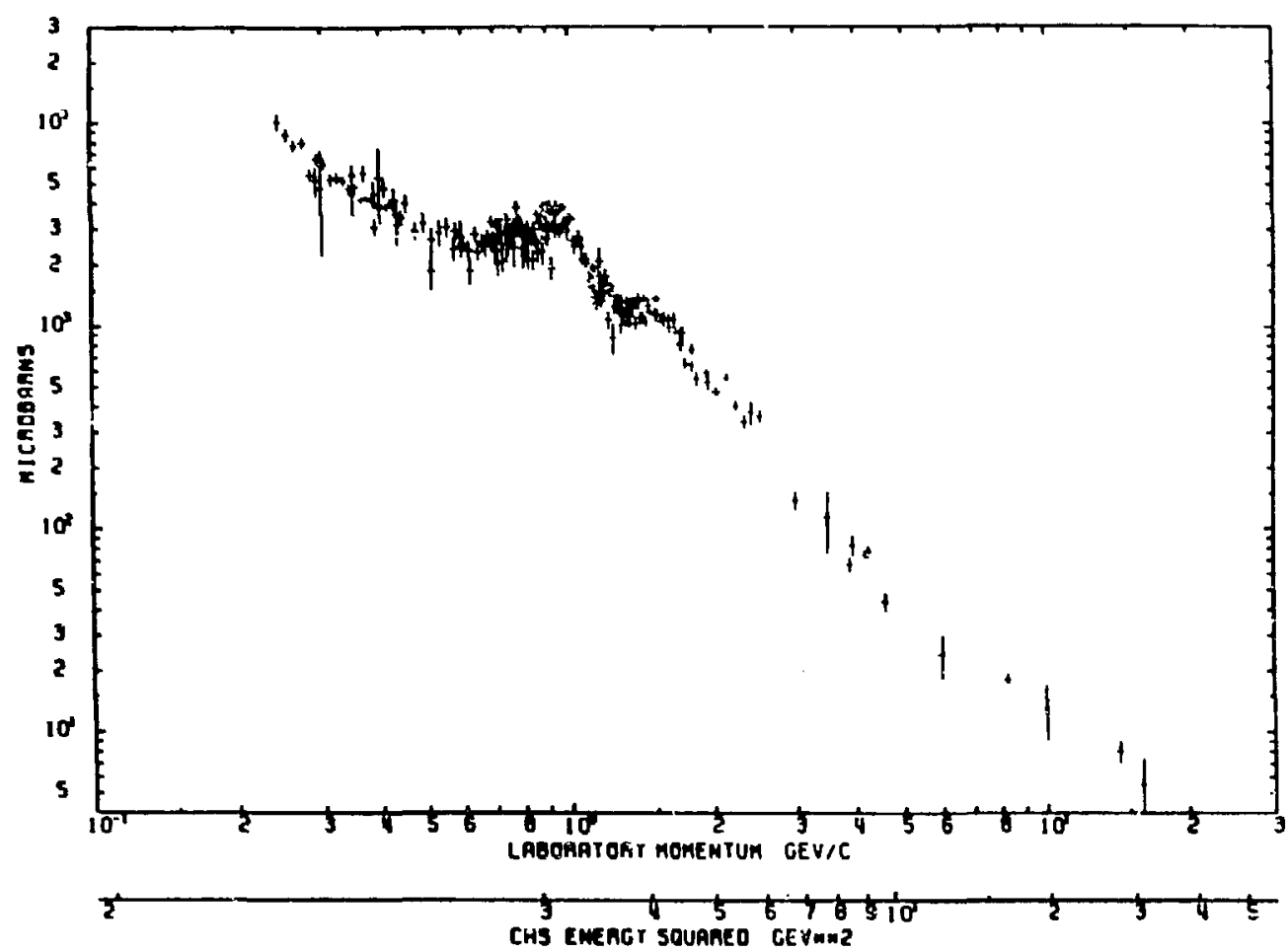

Figure 3.1.a. Total cross sections for the elementary reaction $K^{-}+p \rightarrow \Lambda+\pi^{0}$ (equivalent to $K^{-}+n \rightarrow \Lambda+\pi^{-}$), as a function of $K^{-}$ momentum. Most $\left(\mathrm{K}^{-}, \pi^{-}\right)$studies have been performed at $\mathrm{P}_{\mathrm{K}} \simeq 800$ $\mathrm{MeV} / \mathrm{c}$. 


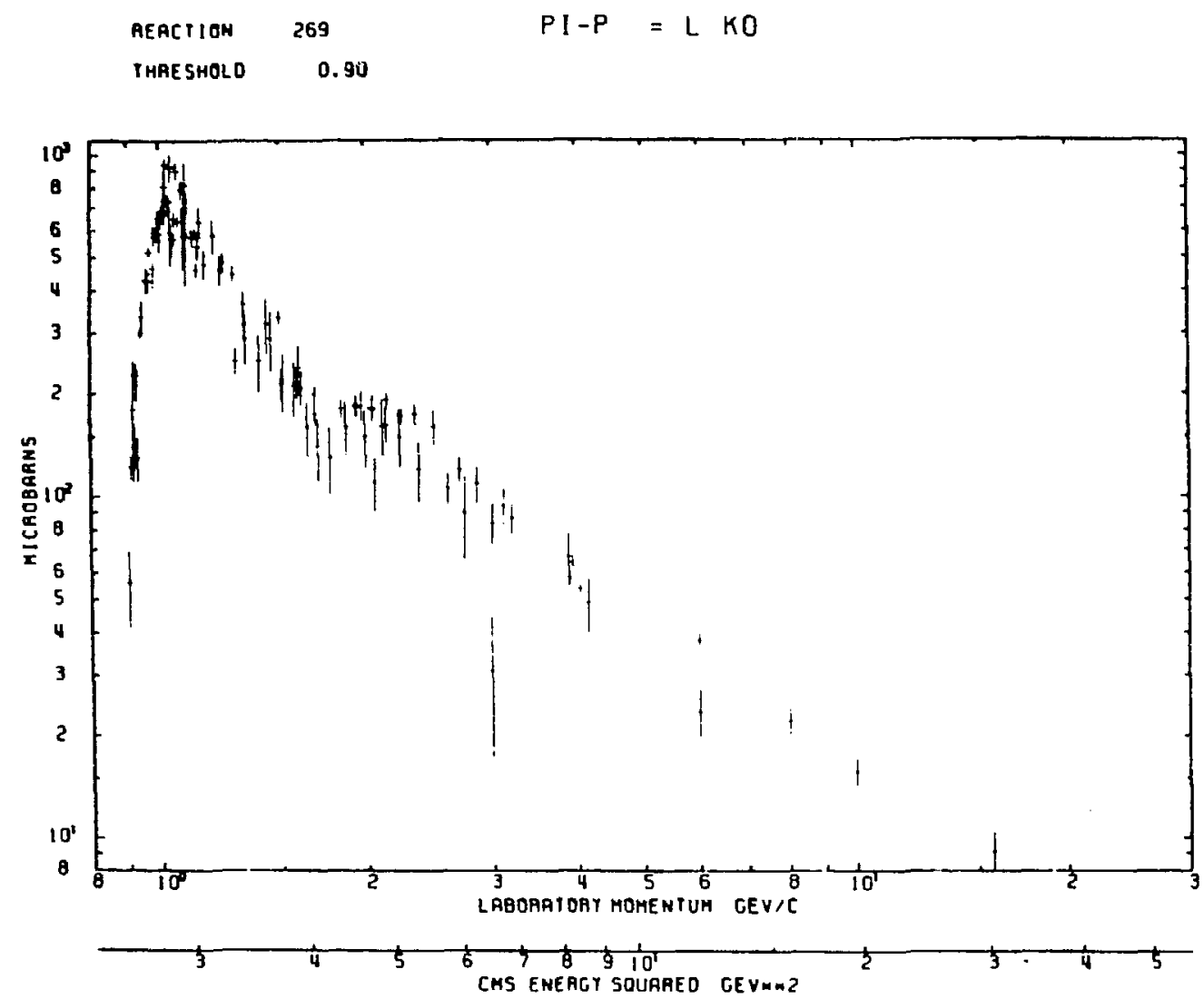

Figure 3.1.b. Total cross sections for $\pi^{-}+p \rightarrow \Lambda+K^{0}$ (equivalent to $\pi^{+} v \mathrm{n} \rightarrow \Lambda+\mathrm{K}^{+}$), as a function of $\pi^{+}$lab momentum. A strong peak structure at $\sim 1050 \mathrm{MeV} / \mathrm{c}$ makes that momentum the obvious beam choice. 
Dover et al. used this two-body cross section distribution, along with other factors in eq. 3.1.a, to estimate the $\pi^{+}$beam momentum maximizing excitation of high spin "stretch states" in a variety of nuclei, including the $\mathrm{J}^{\pi}=2^{+}$states in $\Lambda^{12} \mathrm{C}$ discussed here. The kinematical factor $\alpha$ describes a transformation from the 2-body to the many-body lab system, in which the value of the $\Lambda$ recoil-momentum is altered. This is given (eq. 2.8 in ref. [Do 80]) by $\alpha=1-\left(\beta_{\mathcal{\Lambda}} / \beta_{K}\right)$. The effective number of neutrons available to the reaction, $\mathrm{N}_{\text {eff }}$, for a "stretch state" (eq. 4.7 in [Do 80]) is given by $N_{\text {eff }}=\beta_{J} z^{J} e^{-z}$, where $\beta_{J}=2 / 9$ for the $J=2$, 6-J symbol, $\mathrm{Z}=\mathrm{bq} / 2$ with $\mathrm{b}=1.63 \mathrm{fm}$, a harmonic oscillator parameter, and $q$ is the momentum transfer. $N_{\text {eff }}$ is plotted versus $P_{\pi}$ in figure 3.1.c. Ncte the strong $P_{\pi}$ dependence for $\Lambda^{12} \mathrm{C}$ and also that around $P_{\pi} \simeq 1.1 \mathrm{GeV} / \mathrm{c}, \mathrm{N}_{\text {eff }}$ for $\Lambda^{28} \mathrm{Si}$ and $\Lambda^{12} \mathrm{C}$ is similar.

of the remaining factors, pion decay is not a strong function, since the decay rate is only $\sim 20 \%$ smaller at $1300 \mathrm{MeV} / \mathrm{c}$ than at 700 $\mathrm{MeV} / \mathrm{c}$. Combining the 2 -body cross section, $\alpha$, and $\mathrm{N}_{\text {eff }}$ yields the cross sections plotted in figure 3.1.d. This calculation indicates the optimum event-rate is obtained with $\mathrm{P}_{\pi} \simeq 1.05 \mathrm{GeV} / \mathrm{c}$.

Two major factors were not included in the analysis by Dover et al. The first is "broadening" of the cross section via the neutron Fermi-momentum distribution; this is discussed in section 5.3. In any case, this effect would not alter the cross section peak position, and in fact would make the choice of $P_{\pi}$ less critical. 


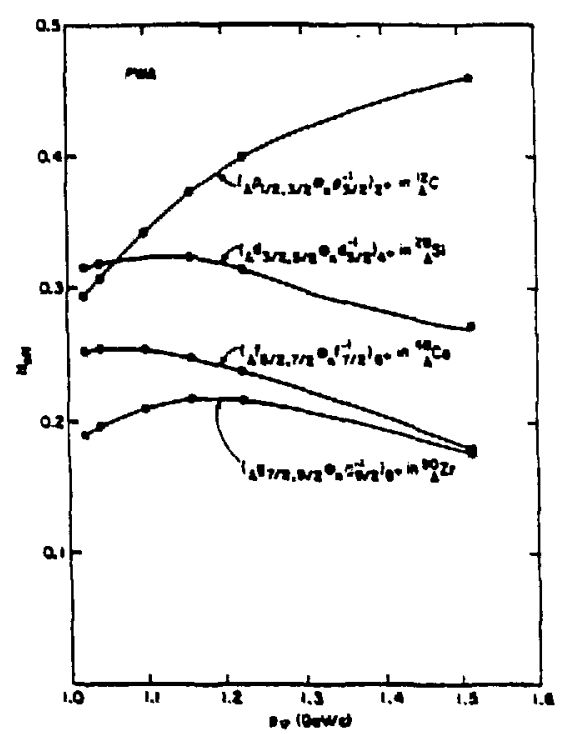

Figure 3.1.c. Effective neutron number $\mathrm{N}_{\text {eff }}$ for excitation to high spin states via the $\left(\pi^{+}, K^{+}\right)$reaction, as a function of pion lab momentum $P_{\pi}$ (from [Do 80]).

However, a second effect, involving a shift in the cross section peak attributable to kinematic factors is potentially critical. A heuristic argument serves to illustrate the situation. The two-body process $\pi^{+}+n \rightarrow \Lambda+K^{+}$threshold of $P_{\pi}=0.89 \mathrm{GeV} / \mathrm{c}$ lowers to $\mathbf{P}_{\pi}=0.57 \mathrm{GeV} / \mathrm{c}$ for the reaction on ${ }^{12} \mathrm{C}$, under the assumption that the reaction occurs coherently, with the entire ${ }^{12} \mathrm{C}$ mass participating kinematically. It follows that the threshold for production of higher mass channels $\left(\mathrm{K}^{*}, \mathrm{Y}^{*}\right.$, etc.) is also lowered. Therefore, by the argument given earlier, that the existence and 


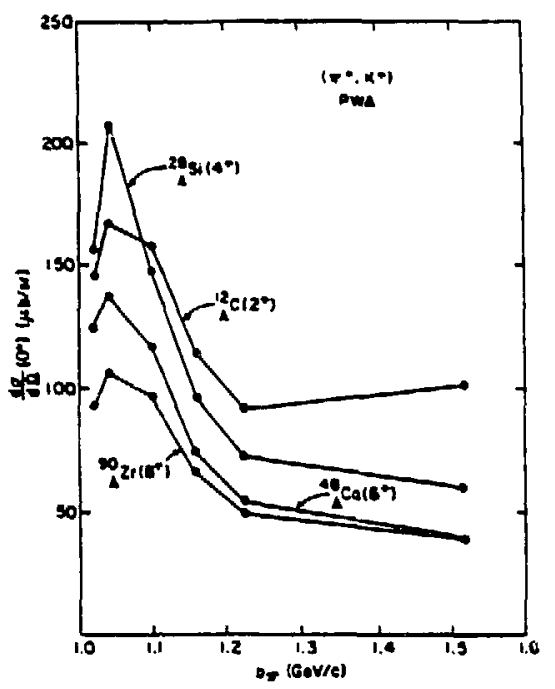

Figure 3.1.d. Differential cross sections at $\theta_{\mathrm{L}}=0^{\circ}$ for axcitation of high spin states in $\Lambda^{12} \mathrm{C}, \Lambda^{28} \mathrm{Si}, \Lambda^{48} \mathrm{Ca}$, and $\Lambda^{90} \mathrm{Zr}$ via the $\left(\pi^{+}, \mathrm{K}^{+}\right)$ reaction, as a function of $P_{\pi}$ (from [Do 80]).

position of the peak in $\pi^{+}+n \rightarrow \Lambda+K^{+}$can be explained by the onset of these channels, the peak in $\Lambda^{12} \mathrm{C}\left(\theta_{\mathrm{L}}=0^{\circ}\right)$ production should shift to a lower momentum, below $P_{\pi}=1.05 \mathrm{GeV} / \mathrm{c}$. In fact, no experimental determination $0 \dot{i}$ the cross section peak as a function of incident momentum has ever been made for a hypernuclear process. If the peak position shifts in the nuclear scattering case, there are several implications for experiment: 1) better flux utilizaton could be made in future experiments ; 2) such an effect might yield information about the interaction -- for example, if the incident $\pi^{+}$ 
mainly interacts with a free neutron, or a neutron imbedded in ${ }^{12} \mathrm{C}$, an $\alpha$, or a deuteron; 3 ) measurement of lowered threshold production might supply evilence for the existence or absence of a strangeness production "hot spot".

In summary, although the proper choice for $P_{\pi}$ is not clearly dictated by analysis, calculations imply $P_{\pi}=1.05 \mathrm{GeV} / \mathrm{c}$ would be reasonable.

\subsection{The AGS}

The optimum experimental $\pi^{+}$beam momentum of $1.05 \mathrm{GeV} / \mathrm{c}$ is above the range of facilities in the "pion factory" class, such as LAMPF, where the maximum available $\pi^{+}$momentum is about $0.67 \mathrm{GeV} / \mathrm{c}$ at the $\mathrm{P}^{3}$ line. However, the high energy proton beams at BNL, CERN, and KEK are adaptable to pion and kaon secondary beam production at intermediate energies suitable for hypernuclear formation, even though this was not originally envisioned as their mission. Pion and kaon beam fluxes at these facilities are sufficient to perform spectroscopy in counter experiments.

Because pions are unstable, with a short ( $26 \mathrm{~ns}$ ) lifetime, they cannot be practically accumulated in storage rings, as for example anti-protons are, but must be used soon after they are produced in proton interactions with nuclei in a production target. The production mechanism is 


$$
\mathrm{p}+\mathrm{A} \rightarrow \mathrm{\pi}^{+}+\text {other }
$$

where "other" includes a possible multitude of particles such as $\pi$, $K$, e, $\mu$, etc., with quantity and type constrained by the total energy available in the collision center-of-momentum and by quantum number conservation. Figure 3.2.a shows $\pi^{+}$total production cross sections versus incident proton momentum in $\mathrm{p}+$ Be collisions. It is seen here that for production optimization, the incident proton energy should exceed at least $\sim 10 \mathrm{Gev}$, because of the sharp rise in the cross section at that energy. But the effective secondary flux is the result of several factors: incident proton momentum, and the cross section for secondary production as a function of outgoing momentum and angle. Figure 3.2.b shows the variation of pion production cross section, in $23.1 \mathrm{GeV} / \mathrm{c} \mathrm{p}-\mathrm{p}$ collisions, as a function of pion momentum. Note the fortuitous maximum at $P_{\pi}$ $\sim 1 \mathrm{GeV} / \mathrm{c}$, coinciding with the desired momentum for the $\left(\pi^{+}, \mathrm{K}^{+}\right)$ reaction. Even with the limitations imposed by beam sharing, the AGS is capable of delivering on the order of $10^{7}$ pions/second to the C2 beam line.

Like other high energy proton accelerators, the BNL Al ternating Gradient Synchrotron (AGS) is composed of distinct accelerator stages, involving different operational concepts. In the first section, protons are accelerated to $200 \mathrm{MeV}$ in an Alvarez-type 


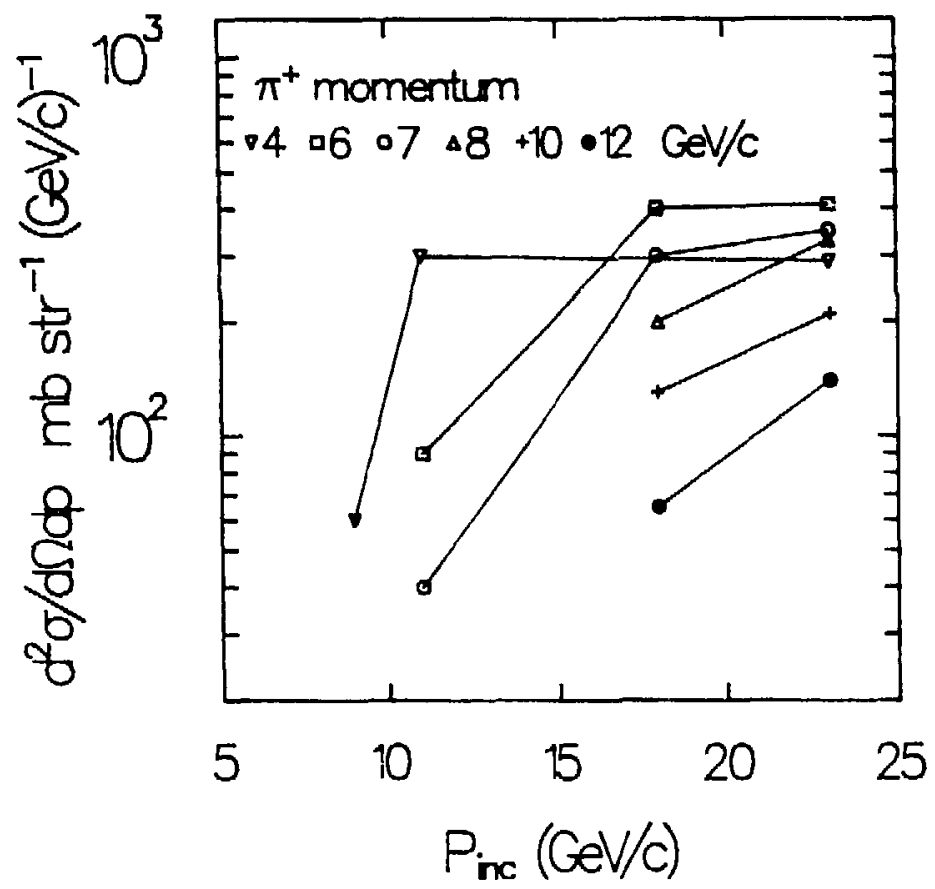

Figure 3.2.a. Production cross sections for $\pi^{+}$as a function of incident proton momenta in $p+B e$ collisions. Note the sharp rise at $P_{p}=10 \mathrm{GeV} / \mathrm{c}$. The figure is from reference [De 65].

linac. This "pre-acceleration" is required so the protons will initially circle the synchrotron with a frequency above the minimum synchrotron radio-frequency accelerating field. After injection into the main $128 \mathrm{~m}$ radius synchrotron ring, the beam containing $-1 \times 10^{13}$ protons is accelerated to $29 \mathrm{GeV}$. The term "alternating gradient" describes the concept used to constrain the phase-space of the beam, known also as "strong-focusing" [Co 52]. The beam passes through magnetic field gradients which alternate in focusing and 


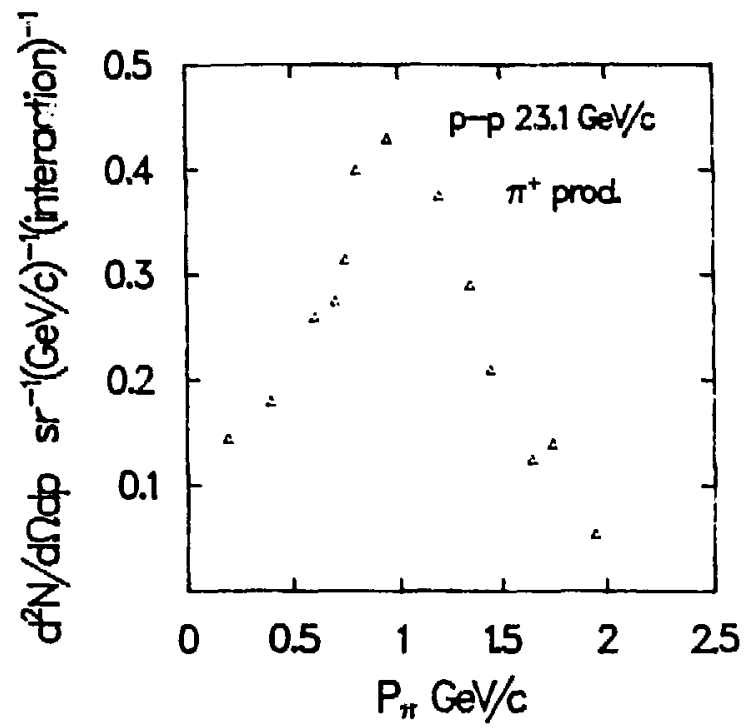

Figure 3.2.b. Production cross sections for $\pi^{+}$as a function of $\pi^{+}$ momenta, in $23.1 \mathrm{GeV} / \mathrm{c}$ p-p collisions. The figure is from [De 65].

defocusing, but have the net effect of focusing, and therefore containing, the beam. The situation is analogous to the optical case of a light beam being focussed by passing through focusing and defocusing lenses of equal strength.

Only a fraction of the entire beam was (and could be) used by the experiment. The beam was split by septum magnets into parts deilvered to the various simultaneously running experiments. In typical $1.5 \mathrm{~s}$ duration primary beam spills, occurring at $2.5 \mathrm{~s}$ intervals, $-2 \times 10^{12}$ protons were incident on the $\mathrm{C} 2-1$ ine $10.16 \mathrm{~cm}$ platinum secondary beam production target. Even though only $\sim 20 \%$ of 
the main beam was directed to the $\mathrm{C} 2$ target, proton $\mathrm{dE} / \mathrm{dx}$ losses in the platinum caused enough melting and sagging to require replacement of the target during the experiment. However, the secondary beam flux of $-4 \times 10^{6} \pi^{+}$particles per spill incident at the scattering target was near the limit imposed by trigger counter dead times.

3.3 Pion beam and hypernuclear spectrometer systems

Magnetic spectrometers are the primary tool of nuclear spectroscopy because the trajectory of a charged particle in a known magnetic field uniquely defines the particle 3-momentum. Once the particle type or mass has been identified by time-of-flight, the 4-momentum is known. Combining this information for all particles involved in a nuclear reaction allows the calculation of the amount of "missing mass" in the reaction, a quantity equivalent to excitation energy. Therefore, detailed and precise knowledge of the magnetic field of the spectrometer is essential to the proper extraction of the nuclear excitation spectrum -- the primary experimental goal.

The mathematical treatment of charged particle trajectory behavior in a magnetic field resembles the description of classical optics, with similar formalism and terminology, except that particle momentum has replaced the quantity of light frequency. A computer 
code embodying this formalism, TR. ORT, used in the design and description of beam transport and spectrometer systems, is discussed in this section.

The remainder of the section is devoted to explaining the operational features of the beam line and spectrometer systems in the language of TRANSPORT. Also, the "floating-wire" technique used to obtain the absolute energy calibration of the magnets is presented.

3.3.1 The mathematical model: TRANSPORT formalism -

The trajectory of a charged particle (known as a ray) in a static magnetic field is defined by the famillar Lorentz force equation

$$
\frac{\partial \vec{p}}{\partial t}=\frac{e}{m}(\vec{v} \times \vec{B})
$$

through integration, given $\vec{B}$ and initial conditions. Equivalently, the momentum may be calculated if the trajectory and field are known. In principle, this equation of motion could be integrated for a multitude of particle initial conditions, reflecting the gross behavior of a particle beam to arbitrary accuracy. This is the strategy of "ray-tracing" type programs [Ko], with the calculation truncated at some point because most particle transport systems 
require only a few multipoles in $\vec{B}$ for effective modeling. However, in applications where a second order calculation is sufficient, and ease and speed of calculation is desired, the TRANSPORT [Br 69] [Br 77] approach is feasible.

In TRANSPORT, the problem is reduced to a process of matrix multiplication representing the phase space coordinates of particle trajectories. The derivation of this formulation proceeds by first eliminating time as an explicit variable in equation $(3.3 .1 . a)$, then writing the solution as a power series. This procedure is discussed in detail elsewhere $[\mathrm{Br} 69]$. The result is cast in a matrix form which is easy to physically interpret, and establishes a language for discussion of magnet and beam optics.

At any point in the system, a charged particle is represented by the column vector

$$
x=\left(\begin{array}{c}
x \\
\theta \\
y \\
\phi \\
\ell \\
\delta
\end{array}\right),
$$

where $x$ is the horizontal displacement from the central trajectory(CT), $\theta$ is the angle of particle trajectory with respect 
to the CT in the horizontal plane, $y$ is the vertical displacement from the $\mathrm{CT}, \phi$ is the angle of particle trajectory with respect to the $\mathrm{CT}$ in the vertical plane, $\ell$ is the path difference from the CT, and $\delta$ is $\Delta p / p$, the deviation from the central momentum. These variables are defined in the curvilinear co-ordinate system schematically shown in figure 3.3.1.a. Every beam line element--magnet, drift space, etc.-- is represented to first order by a square matrix $R$, describing the effect of the element on the passing ray. An initial ray $x_{a}$ is expressed as the final ray $x_{b}$ after traversing element $R_{1}$, written

$$
x_{b}=R_{1} x_{a}
$$

Given a matrix for every one of $n$ elements in a beam line, the final ray $x_{c}$ is:

$$
x_{c}=R_{n} \cdots R_{2} R_{1} X_{a}=R_{t} x_{a}
$$

This procedure is extensible to second order by including a three index matrix $T$ so that components of the final vector are:

$$
x_{i}=\sum_{j} R_{i j} X_{j}+\sum_{j k} T_{i j k} X_{j} X_{k} .
$$

TRANSPORT truncates the solution here at second-order. 


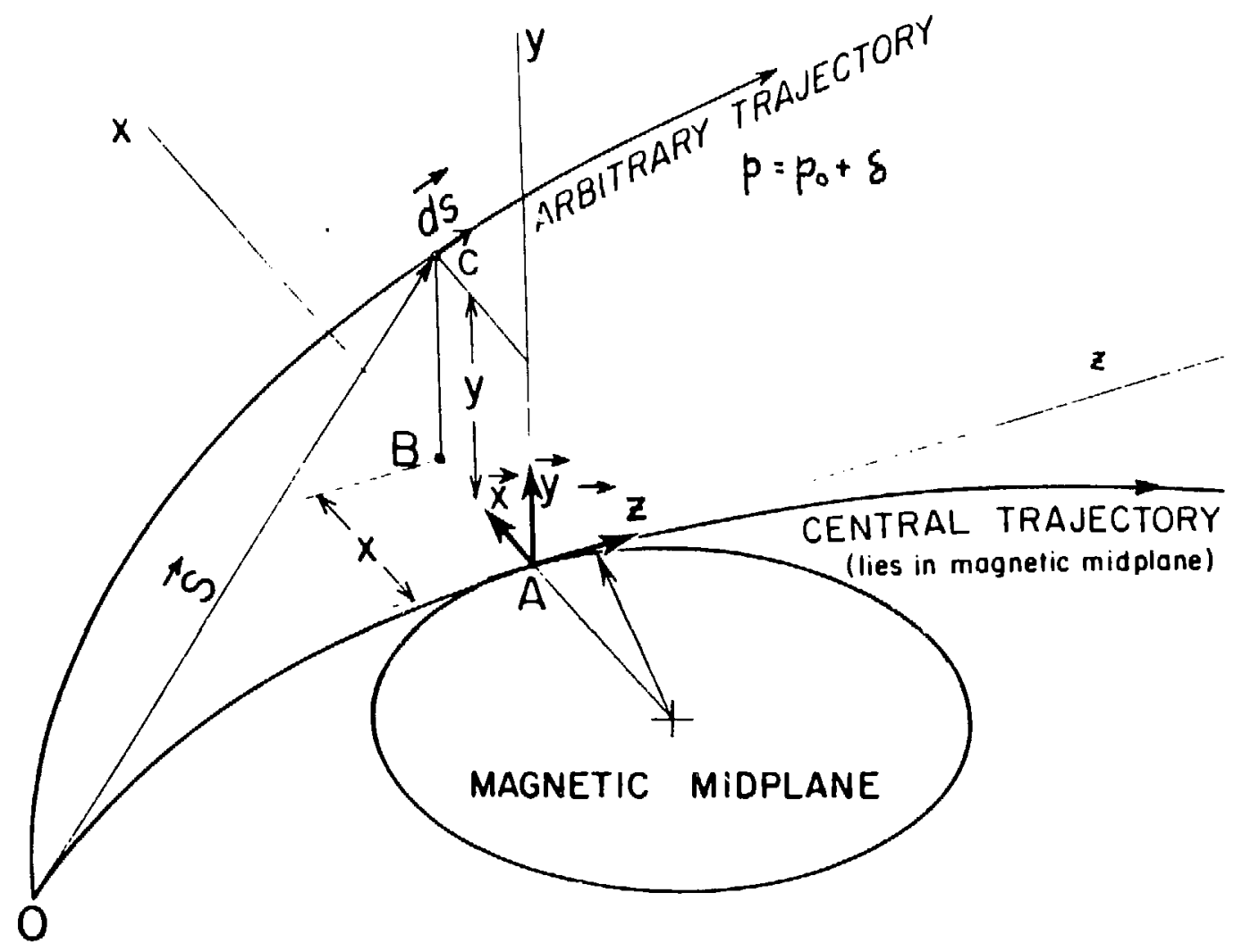

Figure 3.3.1.a. The curvilinear coordinate system in which TRANSPORT variables are defined. 


$$
R=\left(\begin{array}{llllll}
\operatorname{coskL} & \frac{1}{\mathbf{k}} \operatorname{sinkL} & 0 & 0 & 0 & 0 \\
-\mathrm{ksinkL} & \operatorname{coskL} & 0 & 0 & 0 & 0 \\
0 & 0 & \operatorname{coshkL} & \frac{1}{\mathrm{k}} \operatorname{sinhkL} & 0 & 0 \\
0 & 0 & \text { ksinhkL } & \operatorname{coshkL} & 0 & 0 \\
0 & 0 & 0 & 0 & 1 & 0 \\
0 & 0 & 0 & 0 & 0 & 1
\end{array}\right)
$$

Figure 3.3.1.b. Matrix $R$ for a horizontal (x) plane focusing quadrupole magnet, with variables explained in the text.

An example of this construction is the matrix $R$ (figure 3.3.1.b) for a horizontal (x) plane focusing quadrupole magnet shown in figure 3.3.1.c where $\mathrm{L}$ is the effective magnet length, $k^{2}=\left(B_{0} / a\right)\left(1 / B_{\rho}\right)$, a is the radius of the magnet aperture, $B_{0}$ is the field at radius $a$, and $B_{p}$ is magnetic rigidity of the central trajectory $B p=P_{0} / e$. Note that for a vertically focusing ( $y$-plane) quadrupole, the first two submatrices are interchanged. The physical significance of the terms in this matrix become evident when they are identified with coefficient labels used in practice in 


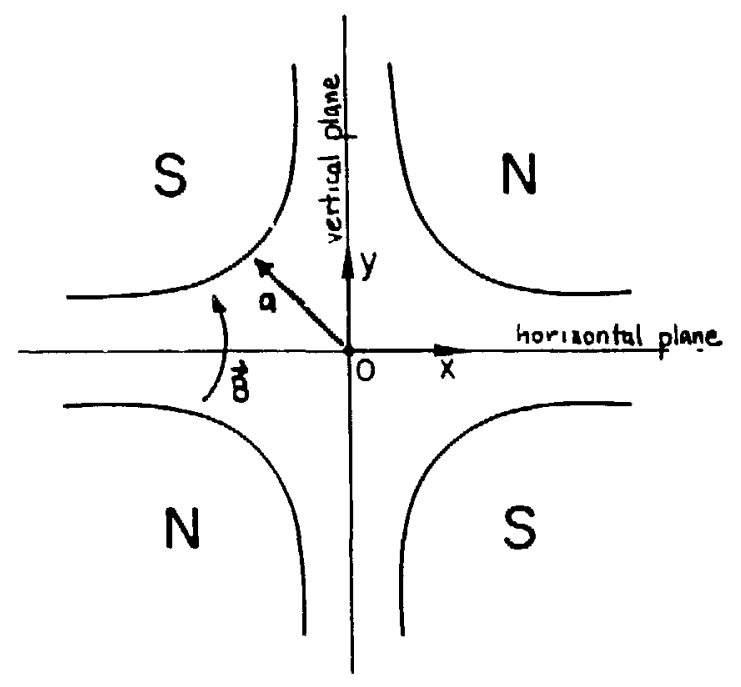

Figure 3.3.1.c. Schematic drawing of a vertically focusing quadrupole magnet, showing pole face orientation, and various dimension labels dicussed in the text.

TRANSPORT (figure 3.3.1.d). Doing the multiplication for the first two components of the final position column vector gives

$$
\begin{aligned}
& x=\left(x \mid x_{0}\right) x_{0}+\left(x \mid \theta_{0}\right) \theta_{0}=(\operatorname{coskL}) x_{0}+\left(\frac{1}{k} \operatorname{sinkL}\right) \theta_{0}, \\
& \theta=\left(\theta \mid x_{0}\right) x_{0}+\left(\theta \mid \theta_{0}\right) \theta_{0}=(-k \operatorname{sinkL}) x_{0}+(\operatorname{coskL}) \theta_{0} \cdot(3.3 .1 . f)
\end{aligned}
$$

Given the initial conditions (for Q9) $x_{0}=10 \mathrm{~cm}, \theta_{0}=10 \mathrm{mr}, B_{0}=10.9 \mathrm{G}$, $a=10.2 \mathrm{~cm}, L=35.6 \mathrm{~cm}, p_{0}=.8 \mathrm{GeV} / \mathrm{c}$, and $\mathrm{k}=1.16$, calculation with equation 3.3.1.f yields $x=-12.1 \mathrm{~cm}$, and $\theta=-6.0 \mathrm{mr}$, 
$R=\left(\begin{array}{llllll}\left(x \mid x_{0}\right) & \left(x \mid \theta_{0}\right) & \left(x \mid y_{0}\right) & \left(x \mid \phi_{0}\right) & \left(x \mid l_{0}\right) & \left(x \mid \delta_{0}\right) \\ \left(\theta \mid x_{0}\right) & \left(\theta \mid \theta_{0}\right) & \left(\theta \mid y_{0}\right) & \left(\theta \mid \phi_{0}\right) & \left(\theta \mid l_{0}\right) & \left(\theta \mid \delta_{0}\right) \\ \left(y \mid x_{0}\right) & \left(y \mid \theta_{0}\right) & \left(y \mid y_{0}\right) & \left(y \mid \phi_{0}\right) & \left(y \mid l_{0}\right) & \left(y \mid \delta_{0}\right) \\ \left(\phi \mid x_{0}\right) & \left(\phi \mid \theta_{0}\right) & \left(\phi \mid y_{0}\right) & \left(\phi \mid \phi_{0}\right) & \left(\phi \mid l_{0}\right) & \left(\phi \mid \delta_{0}\right) \\ \left(l \mid x_{0}\right) & \left(l \mid \theta_{0}\right) & \left(l \mid y_{0}\right) & \left(l \mid \phi_{0}\right) & \left(l \mid l_{0}\right) & \left(l \mid \delta_{0}\right) \\ \left(\delta \mid x_{0}\right) & \left(\delta \mid \theta_{0}\right) & \left(\delta \mid y_{0}\right) & \left(\delta \mid \phi_{0}\right) & \left(\delta \mid l_{0}\right) & \left(\delta \mid \delta_{0}\right)\end{array}\right)$

Figure 3.3.1.d. General coefficient labels for the first-order R-matrix used in TRANSPORT. This is the same matrix as in fig. 3.3.1.b.

demonstrating a change in the trajectory by focusing in the $x$-plane. Note that a ray having $x_{0}=0$, and $\theta_{0}=0$, the initial conditions of the central trajectory ray, is not deflected by the quadrupole. Also, the element $\left(x \mid \delta_{0}\right)=0$, meaning the quadrupole does not introduce any dispersion into the beam.

Rather than tediously catalogue examples of magnetic lenses, it is better now to discuss the physical significance of elements in the general first-order coefficient matrix $R$, represented in figure 3.3.1.d. 
The first order spatial dispersion of a system. $d_{x}=R_{16}=\left(x \mid \delta_{0}\right)$, is generated only in elements where the central trajectory may be deflected, such as in a dipole magnet. The $x$-position after a dipole is (to first-order)

$$
x=\left(x \mid x_{0}\right) x_{0}+\left(x \mid \theta_{0}\right) \theta_{0}+\left(x \mid \delta_{0}\right) \delta_{0} \quad \cdot(3.3 .1 \cdot g)
$$

Suppose that for a group of rays (i. e., a beam) $x_{0}=\theta_{0}=0$, but that $\delta_{0}$ takes on a range of values $\left(-\delta_{0},+\delta_{0}\right)$. Then the dipole acts to disperse the initially spatially compact beam in a range of x-positions, like a prism disperses white light into color components.

The first order angular dispersion $d_{x}^{\prime}=R_{26}=\left(\theta \mid \delta_{0}\right)$, is viewed similarly by considering $\theta$ after a dipole,

$$
\theta=\left(\theta \mid x_{0}\right) x_{0}+\left(\theta \mid \theta_{0}\right) \theta_{0}+\left(\theta \mid \delta_{0}\right) \delta_{0} \cdot(3.3 .1 . h)
$$

For the same conditions given above, the meaning of the angular dispersion is also apparent.

Categpries of beam imaging conditions are also simple to outline in first order. Point-to-point imaging in the $x$-plane occurs when $x=x_{0}$ (that is, $\left(x \mid x_{0}\right)=1$ ), for any value of $\theta_{0}$, implying $R_{12}=\left(x \mid \theta_{0}\right)=0$. The parallel-to-point imaging condition $R_{11}=\left(x \mid x_{0}\right)=0$ means that a parallel beam will be made to converge at a point. 
Conversely, the point-to-parallel imaging condition is $R_{22}=\left(\theta \mid \theta_{0}\right)=0$. Imaging constraints in the y-plane are written in a similar fashion. The power of the TRANSPORT program lies in the ability to fit optical parameters, such as field strengths, constrained by a variety of $R$-matrix requirements, including desired imaging. Thus TRANSPORT has two uses: 1) the calculation of the R-matrix and T-matrix, given data on the beam line elements; and conversely, 2) the calculation of parameters required of a beam line to meet desired optical imaging conditions. The operational characteristics of the beam line and spectrometer systems will now be discussed from the viewpoint of the currently well understood TRANSPORT calculations.

\subsubsection{Experimental system overview}

The primary components of the experimental apparatus are shown in figure 3.3.2.a. There are three main sections: 1) the pion beam line (Low Energy Separated Beam line or LESB-I), from the platinum secondary beam production target to the mass slit, 2) the pion spectrometer (Sp1) from 05 to 07 , and 3) the kaon spectrometer (Sp2) from 08 to 011 . Note that in the discussion of the three main systems, the distance scale, $z$, is maintained separately, e.g. is not accumulated for the overali system. 


\section{C2 BEAM LINE AND SPECTROMETERS}

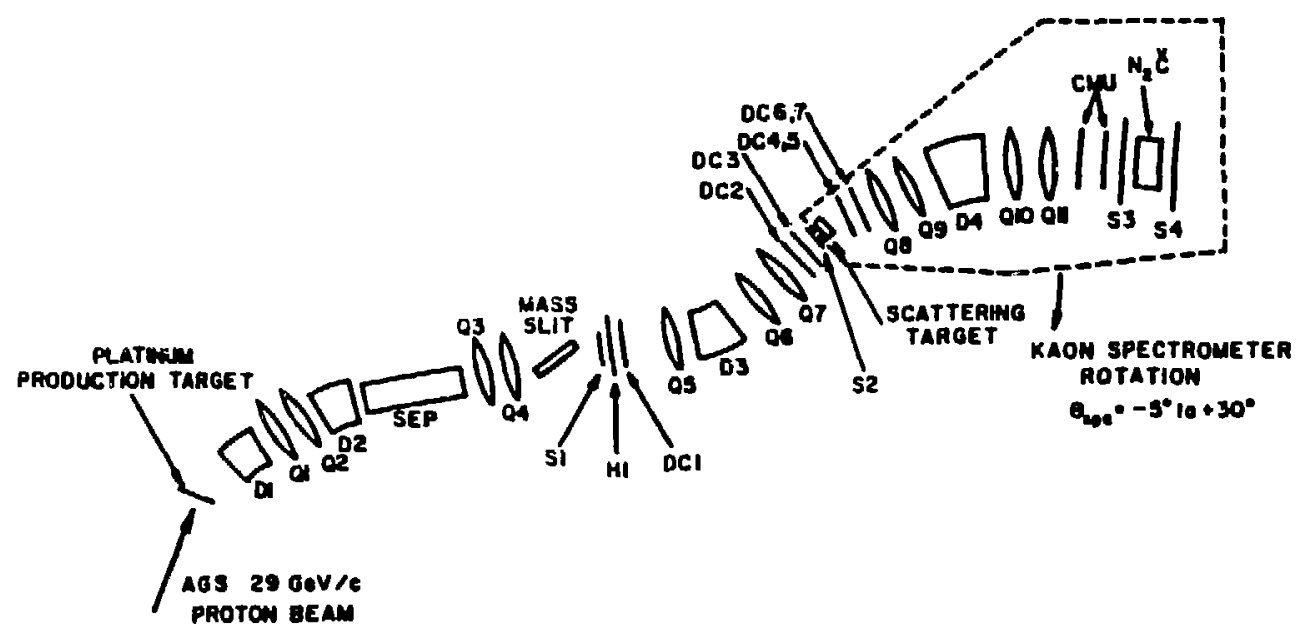

Figure 3.3.2.a. Primary components of the experimental apparatus. Quadrupoles are indicated by convex lens shapes while dipoles are represented by wedge shapes. The other elements are various detectors, described in the text. 
Secondary particles produced in the platinum target were collected at an angle of 10 degrees from the incident proton beam. The purpose of the LESB-I was to select a desired particle species (pion or kaon) component of this secondary beam, using an $\vec{E} \times \vec{B}$ velocity selector, and focus it on a gap in the mass slit. Individual particles in this purified beam were then identified by time-of-flight (TOF) between scintillators $S 1$ and $S 2$, and then momentum analyzed by calculating their deflection in the system from positions in drift chambers DC1, DC2, and DC3. After interacting in the scattering target, particles in $\mathrm{Sp} 2$ were identified by TOF between S2 and S3, with momentum analysis derived from positions in DC4, DC5, DC6, DC7 and proportional chambers labelled CMU.

\subsubsection{The LESB-I beam line}

The principal function of the LESB-I was to provide a purified beam of charged pions or kaons. Since the many different experiments performed at the $\mathrm{C} 2$ beam line require beams of different particles and momenta, the LESB-I was designed to supply beams in a wide variety. For the current experiment, both $\pi^{+}$and $\mathrm{k}^{+}$beams at $716 \mathrm{MeV} / \mathrm{C}$ and $1054 \mathrm{MeV} / \mathrm{c}$ were used. The accurate tuning of the LESB-I required knowledge of its optical properties. TRANSPORT input data for LESB-I are included in appendix 3.3.3.a. The general 
imaging condition in LESB-I was point-to-parallel-to-point, as the beam proceded from production target to separator to mass slit. Collecting the secondary beam at an angle of 10 degrees from the incident proton beam, dipole septum magnet D1 crudely selected a momentum bite of $\pm 5 \%$ in bending the beam through an angle of 11.5 degrees. The quadrupole doublet 0102 served to confine the beam by focusing it to be parallel in $y$ and slightly diverging in $x$. This condition was required by the geometry of the next element, the $\vec{E} \times \vec{B}$ velocity selector known simply as the "separator". The operating principle of the separator was elementary: in a region of perpendicular $\vec{E}$ and $\vec{B}$ fields, the trajectory of paticles with velocity $\vec{v}=E / B$ is not deflected. In the LESB-I, the $\vec{E}$ field was maintained at $550 \mathrm{kV}$ across a 4 inch gap formed between two plates in t. lane. The $\vec{B}$ field, generated by a dipole with pole faces in the $y$-plane, was varied in order to select the desired velocity. The separator was $324.4 \mathrm{~cm}$ long, so the parallel beam condl_.on was necessary tn prevent loss of the beam in collisions with the separator walls. Also, the angular separation of mass species by the quadrupole doublet 0304 was more effective for an initially parallel beam.

Figure 3.3.3.a summarizes the evolution of the beam profile in $x$ and $y$ as a function of distance over the length of the beam line, illustrating the behavior in the separator $(\sim 4 \mathrm{~m}$ to $-7.5 \mathrm{~m})$ and the focusing effects of the quadrupole doublets. The second quadrupole 


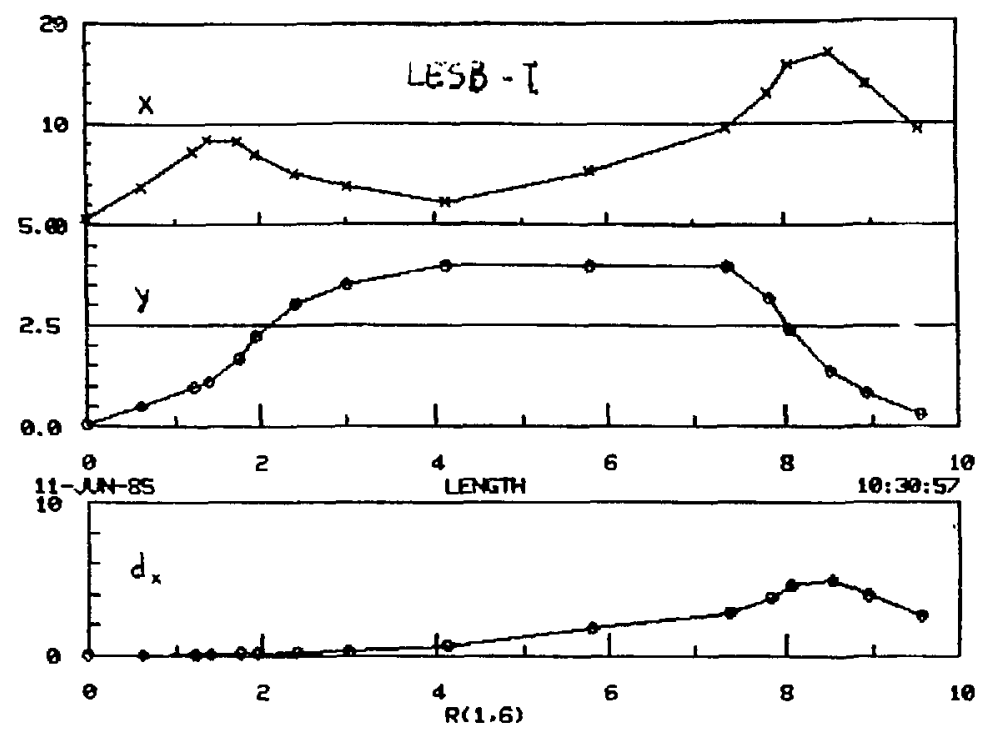

Figure 3.3.3.a. Beam profile in the LESB-I as a function of distance (meters) for: $x$-plane (top), y-plane (middle), and divergence in $\mathrm{x}$ (bottom).

doublet, 0304, created a dispersed double focus at the mass slit, where the final beam purification occurred. Figure 3.3.3.b shows how the separated mass species were incident on the mass slit; data were collected for both pion beam and kaon beam triggers over a range of $\mathrm{BO}$ magnet settings. The tails of species distributions overlap, so that the beam is not entirely pure. Another property of the mass slit separation arrangement was that the rejected beam scattered into the mass slit access "cave" showering nearby detectors with background particles. This was a significant source 


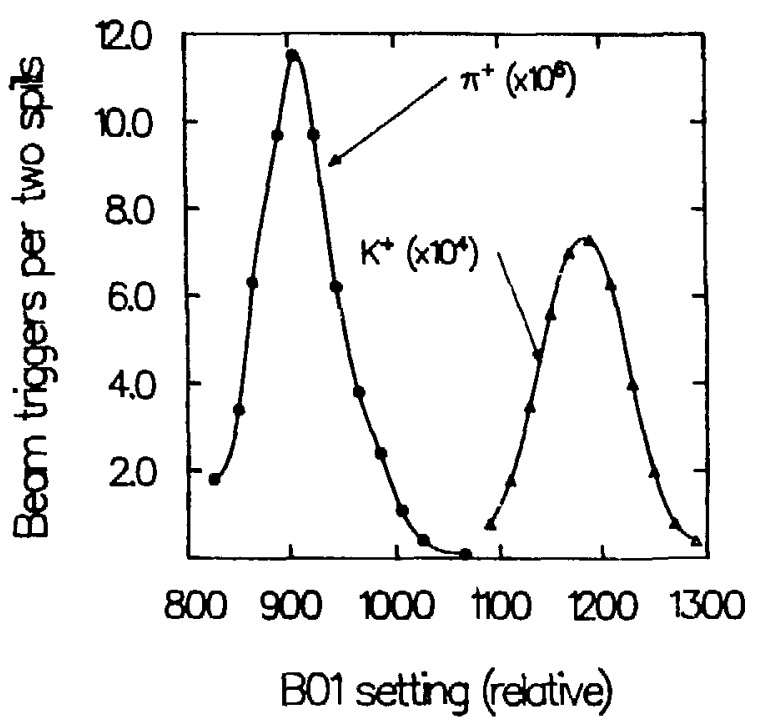

Figure 3.3.3.b. Pion beam and kaon beam triggers (transmitted through Sp1) as a function of separator magnet (B01) setting.

of spurious single-count rates in the detectors DC1, S1, and $H 1$ (hodoscope).

LESB-I was used to provide either a $\pi^{+}$beam $(\sim 1000: 1 \pi: \mathrm{K}$ ratio) or a $\mathrm{K}^{+}$beam $(-15: 1 \quad \pi: \mathrm{K}$ ratio $)$. Both beams were used with spectrometers set for elastic scattering at $716 \mathrm{MeV} / \mathrm{c}$ and 1054 $\mathrm{MeV} / \mathrm{c}$. This allowed calibration of the momentum setting in $\mathrm{Spl}$ by knowing the momentum setting of $\mathrm{Sp} 2$ from a wire orbit study. Then the beam line could be accurately set at the incident $\pi^{+}$momentum of $1054 \mathrm{MeV} / \mathrm{c}$ required for the $\left(\pi^{+}, \mathrm{K}^{+}\right)$reaction. Also, extensive studies of TRANSPORT type optical coefficients for Sp1 and Sp2 at 
near elastic scattering conditions required flexibility of the beam line. Spectrometers $S p 1$ and $S p 2$ and their calibration will be discussed in the following three sections.

\subsubsection{The pion spectrometer -- Sp1}

As discussed earlier, the incident pion beam momentum that maximizes hypernuclear production in the $A\left(\pi^{+}, \mathrm{K}^{+}\right) \wedge^{*}$ reaction is expected to be about $1050 \mathrm{MeV} / \mathrm{c}$. The experimental problem was to produce a beam in that momentum range and measure the momentum of individual pions in the beam. The momentum of the pion is calculable if the following quantities are know 1) the central momentum of the spectrometer dipole; 2 ) an optical description (elements of matrices $R$ and $T$ ) of the system with which to calculate $\delta$; 3$)$ position at one end, and position and angle at the other end of the spectrometer; and 4) TOF identification of particle type. The acquisition of this information is discussed in this section. The name Sp1 refers to the spectrometer from elements DC1 to S2 (figure 3.3.2.a), a nomenclature intended to avoid confusion since it is not exclusively a pion spectrometer.

The central momentum of Sp1 was not measured directly, but by reference to the known (from wire orbit studies, discussed in section 3.3.5) central momentum of $\mathrm{Sp2}$, the kaon spectrometer. With $\mathrm{Sp} 2$ set to a known momentum value, no scattering target present, and 
Sp2 set at zero degrees (so-called "straight-through" condition), the dipole field in Sp1 was varied until the beam profile was centered at the rear of Sp2. Under these conditions, the central momenta of Sp1 and Sp2 were equal. The setting in Sp1 was reproducible independent of this method because a hall probe was used to monitor the field in dipole D3. Hall probe versus momentum calibration tables for various magnets in the system are included in appendix 3.3.4.a.

Because dispersion in Sp1 was primarily in the $x$-plane, $\delta$ (to first-order) was given by

$$
\delta=\frac{1}{R_{16}}\left[x_{f}-R_{11} x_{r}-R_{12} \theta_{r}\right],
$$

where $f$ and $r$ refer to front (at DC1) and rear (at DC2,3) of Sp2, and $R_{11}, R_{12}$, and $R_{16}$ are first-order TRANSPORT coefficients. The TRANSPORT input data, together with a completed calculation for $\mathrm{Sp} 1$ are include in appendix 3.3.4.b. Figure 3.3.4.a shows the evolution of the beam profile in Sp1. Note the focus in $y$, and the slight divergence in $x$ at the end, near the scattering target. The design imaging condition was a "waist", covering the target.

Not all unwanted beam particles could be "tuned-out" of the beam in the separator-mass-slit stage -- kaon beams typically contain $\sim 15$ times as many pions as kaons, while pion beams contain primarily pions, with other particle contaminants. Therefore, the 


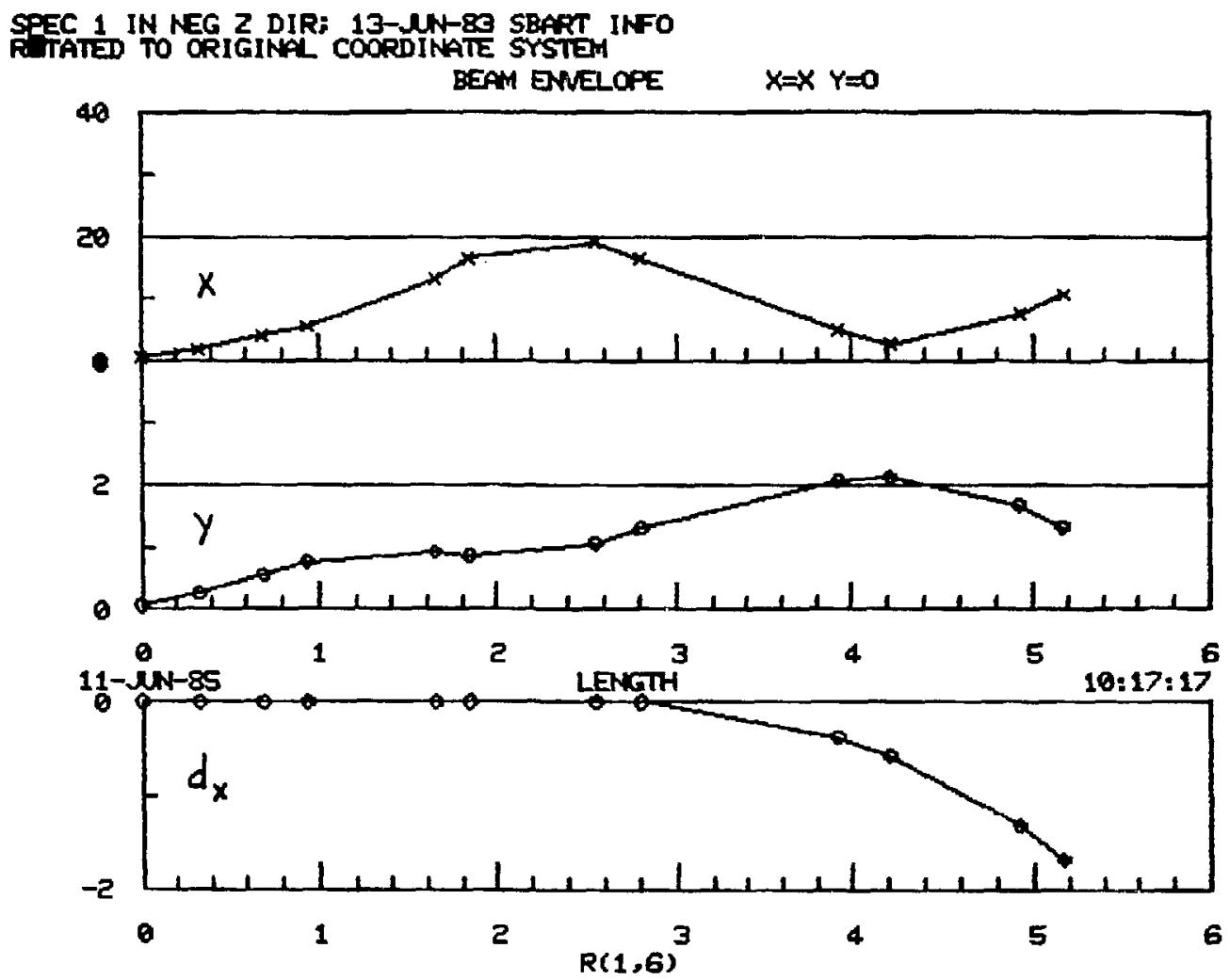

Figure 3.3.4.a. Evolution of the beam as it passes through $S p 1$. Note the waist at the end of the system, confining the beam to the scattering target volume. 
flight path in Sp1 must be sufficiently long to separate pions and kaons by 1 ns (the typical time resolution) in TOF over a wide range of beam momenta $(700$ to $1100 \mathrm{MeV} / \mathrm{c})$. In figure 3.3.4.b, data taken from the "Qvt" time bin recorder shows $p, K^{+}$, and $\pi^{+}$components of the $\pi$-beam at $\mathrm{P}_{\text {bean }}=1054 \mathrm{MeV} / \mathrm{c}$, in an S1-to-S2 TOF spectrum. The kaon peak was 15 ns from the pion peak, and was therefore separable in fast electronic circuitry. For the $\pi$-beam tune, the percentage of $\mathrm{p}$ and $\mathrm{K}^{+}$particles was only about $2 \%$ of the total beam flux. A positron contamination of the $\pi^{+}$peak of about $30 \%$, which is not visible in figure 3.3.4.b because the TOF was too short over the S1-S2 path at $\mathrm{P}_{\text {beam }}=1054 \mathrm{MeV} / \mathrm{C}$, is discussed in section 4.2.

The hodoscope ejement $\mathrm{H} 1$ was used in coincidence with DC1 to reduce in software the considerable singles rate in DC1 associated with background in the mass-slit cave.

\subsubsection{The kaon spectrometer -- Sp2}

For an incident pion of $\mathrm{P}_{\text {beam }}=1054 \mathrm{Mev} / \mathrm{c}$, kaons emitted in the formation of the ground state in $\Lambda{ }^{12} \mathrm{C}$ have $P_{K}=716 \mathrm{MeV} / \mathrm{c}$. The mass of $\wedge^{12} \mathrm{C}$ is

$$
m\left(\Lambda^{12} C\right)=m\left({ }^{12} C\right)+m(\Lambda)-m(n)+B E(n)-B E(\Lambda) \cdot e q \cdot 3 \cdot 3 \cdot 5 \cdot a
$$

In this expression, $m(\Lambda)=1115.6 \mathrm{MeV}$, and $m(n)=939.6 \mathrm{MeV}$. The 


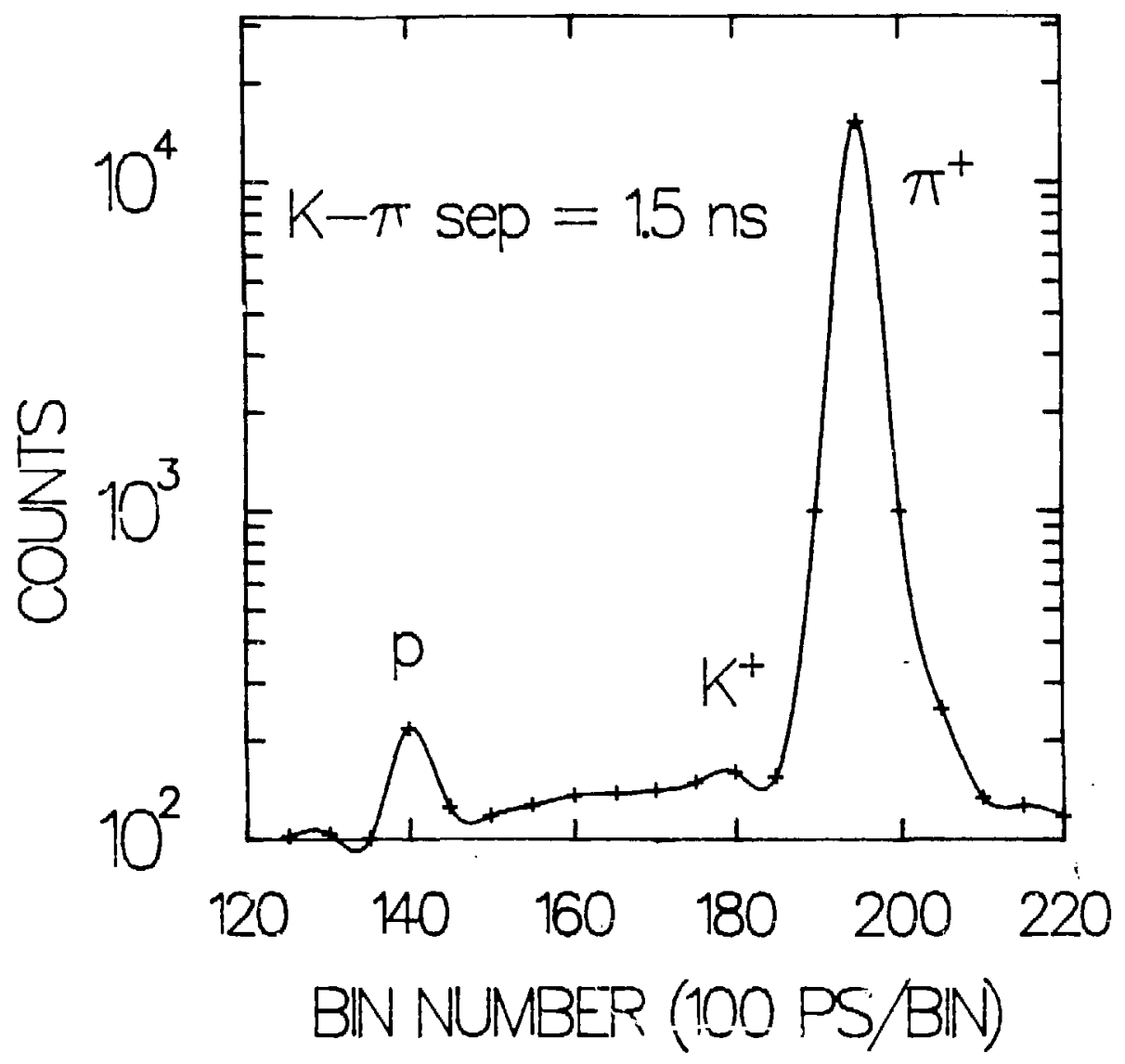

Figure 3.3.4.b. TOF separation of the pion beam in Spl at 1054 $\mathrm{MeV} / \mathrm{c}$, showing $\mathrm{p}, \mathrm{K}^{+}$, and $\pi^{+}$components. 
binding energy of the neutron, $B E(n)=18.7 \mathrm{MeV}$, is known from the reaction threshold for ${ }^{12} \mathrm{C}(\gamma, \mathrm{n})^{11} \mathrm{c}$, and the $\Lambda$ binding energy, $\mathrm{BE}(\Lambda)=10.9 \mathrm{MeV}$, is known from emulsion studies [Bo 70]. These values give

$$
\mathrm{m}\left({ }_{\Lambda}{ }^{12} \mathrm{C}\right)=\mathrm{m}\left({ }^{12} \mathrm{C}\right)+183.8 \mathrm{MeV}, \quad \text { eq.3.3.5.b }
$$

equivalent (for kinematic purposes) to viewing the hypernucleus $\Lambda^{12} \mathrm{C}$ as a ${ }^{12} \mathrm{C}$ nucleus excited by $183.8 \mathrm{MeV}$. The commonly used relativistic kinematics program, KINREL, lacks the ability to conserve strangeness in input reactions, so hypernuclei must be treated as excited nuclei. The reaction ${ }^{12} \mathrm{C}\left(\pi^{+}, \mathrm{K}^{+}\right){ }^{12} \mathrm{C}$, with excitation energy $183.8 \mathrm{MeV}$, was used as KINREL input, yielding $715.8 \mathrm{MeV} / \mathrm{c}$ for the outgoing kaon momentum at $\theta_{\mathrm{Lab}}=10^{\circ}$. The magnetic fields in all elements in Sp2 were monitored by Hall-effect probes, and the probe voltages corresponding to various momentum settings are tabulated in appendix 3.3.4.a. Most of the ${ }^{12} \mathrm{C}\left(\pi^{+}, \mathrm{K}^{+}\right){ }_{\Lambda}^{12} \mathrm{C}$ data were taken with $\mathrm{Sp} 2$ set at $\mathrm{P}_{\mathrm{Sp} 2}=716 \mathrm{MeV} / \mathrm{c}$.

The optical characteristics of $\mathrm{Sp} 1$ and $\mathrm{Sp} 2$ were different because of the type of beam each spectrometer transported. In the case of Sp1, a small phase space in the vertical direction and larger phase space in the horizontal direction was accepted from the mass slit to be refocused in a small volume at the scattering target. In contrast, Sp2 accepted scattered beam with a large phase 
space in both the vertical and horizontal directions, and dispersed it over $x$ and $y$ in large chambers at the rear. Therefore, the quadrupole magnets in $\mathrm{Sp} 2$ were set at a relatively higher field to produce a convergence in $y$ in the region of the dipole magnet, in order to confine the beam within the vacuum system.

Dispersion in the $y$-direction in $\mathrm{Sp} 2$ was minimized in first-order, so that Sp2 is described by eq. 3.3.4.a, like Sp1. The TRANSPORT input deck for Sp2 is included in appendix 3.3.5.a. In figure 3.3.5.a, the beam profile in Sp2 is plotted as a function of distance. Note the y-convergence beginning at $z=2.2 \mathrm{~m}$, at $\mathrm{D} 4$, and the increased dispersion in $x$, beginning at about $z=6.2 \mathrm{~m}$, at the rear of Sp2, between $\mathrm{P} 3$ and $\mathrm{P} 4$.

At $\mathrm{P}_{\mathrm{Sp} 2}=716 \mathrm{MeV} / \mathrm{c}, \mathrm{TOF}_{\mathrm{K}}-\mathrm{TOF}_{\pi}=0.66 \mathrm{~ns} / \mathrm{m}$, so that the $\mathrm{\pi}^{+}$ and $\mathrm{K}^{+}$components of the scattered beam should be separated in TOF by $4.95 \mathrm{~ns}$ over the $7.5 \mathrm{~m}$ path between $\mathrm{s} 2$ and $\mathrm{s} 3$. In figure 3.3.5.b, a TOF spectrum between S2 and S3, pion and kaon groups are separated by about 5 ns ( 50 ps/division). The TOF histogram shown here appears free of background, but that is because test 117, which all these events have passed, includes many cuts requiring proper vertex projection, sensible drift chamber information, etc. In figure 3.3.5.c, however, these cuts are not present, and the kaon signal is not discernable in the background. 
TEE-83) KAON Spectrometer- 12030,12016,18066, 12060,8024

RTATED TO ORIGINAL COORDINATE SYSTEM

BEPM ENVELPE $X=X Y=0$

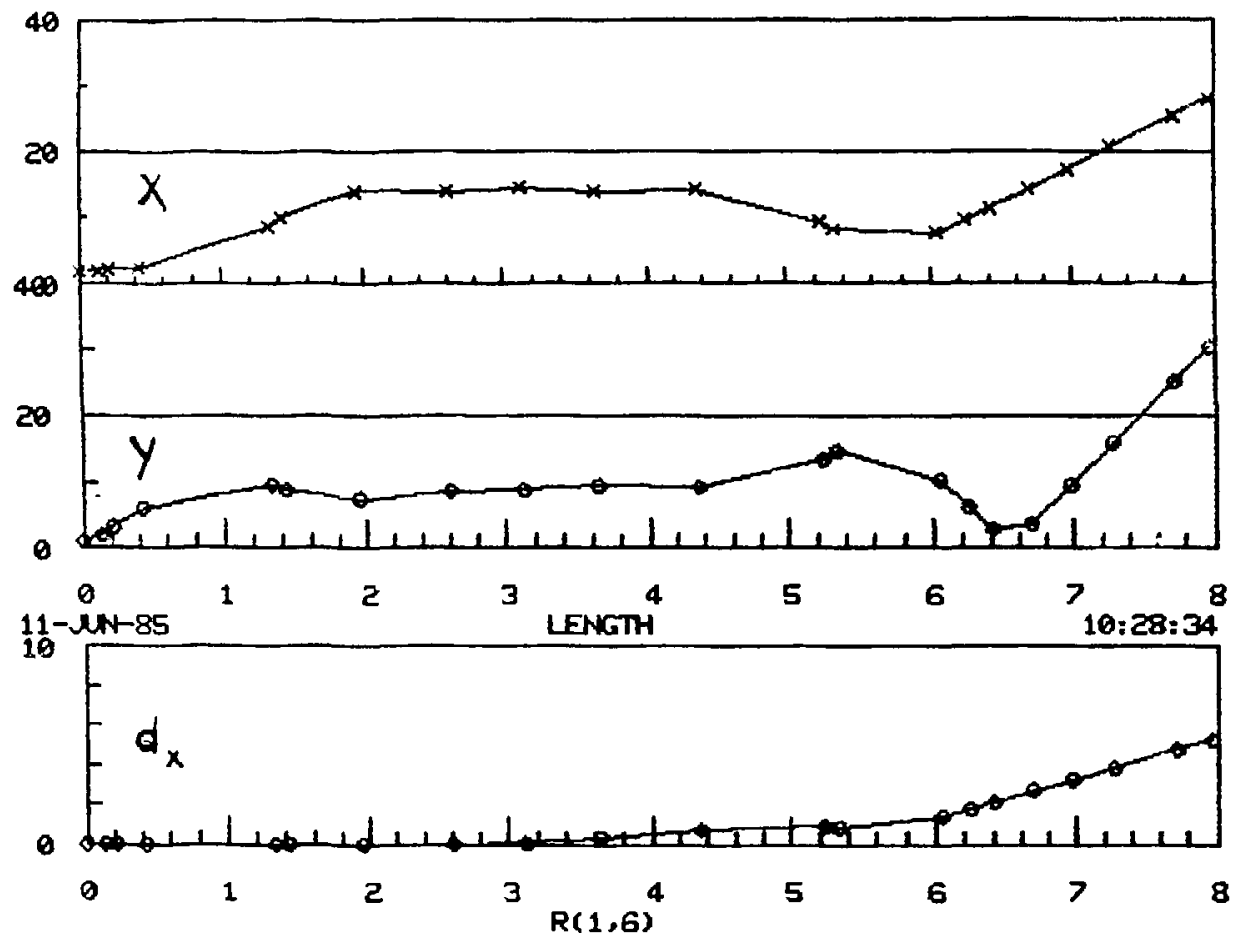

Figure 3.3.5.a. Beam profile evolution in Sp2.

The pions in Sp2 had a jigher relativistic velocity than the kaons. This fact was exploited in using a liquid nitrogen $\left(\mathrm{LN}_{2}\right)$ filled threshold Cherenkov light detector (an array of 7 PMTs surrounding an $\mathrm{LN}_{2}$ filled cavity) at the rear of $\mathrm{Sp} 2$ to distinguish pions from kaons. The threshold condition for the emission of Cherenkov radiation is 


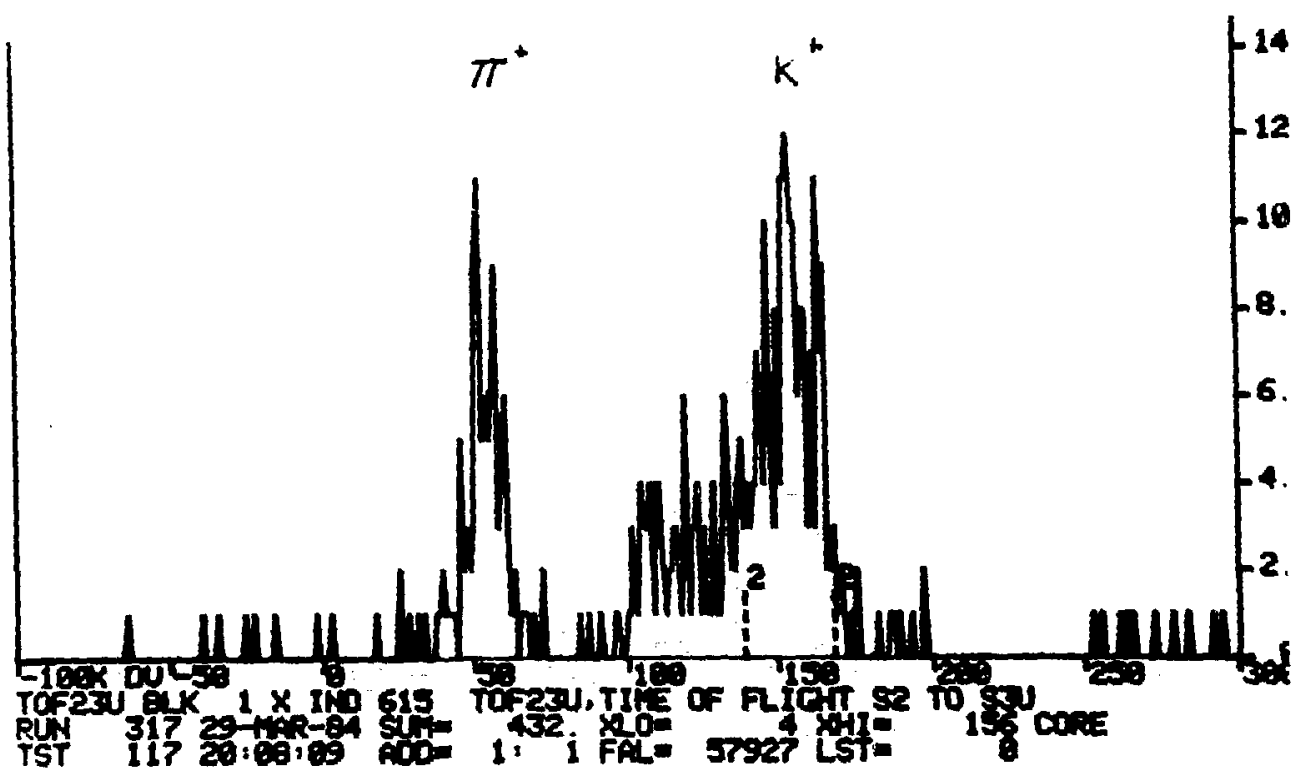

Figure 3.3.5.b. TOF spectrum in Sp2, showing only events which pass all good $\left(\pi^{+}, \mathrm{K}^{+}\right)$tests (test 117 ), except TOF (gate 2).

$$
\beta>\frac{1}{n} \text {, }
$$

eq. 3.3.5.c

where $\beta=v / c$, the relativistic velocity, and $n$ is the index of refraction of the medium. For liquid nitrogen, $n=1.205$, implying particles with $\beta>.83$ are above the emission threshold. For kaons, $\beta=.84$ for $P_{K}=765 \mathrm{MeV} / \mathrm{c}$, while for pions, $\beta=.84$ for $P_{\pi}=217$ $\mathrm{MeV} / \mathrm{c}$. With Sp2 set to transport particles with $\mathrm{P}=720 \pm 4 \% \mathrm{MeV} / \mathrm{c}$, a bur: of light in the $L_{2}$ detected by at least 5 of 7 PMTs in the 


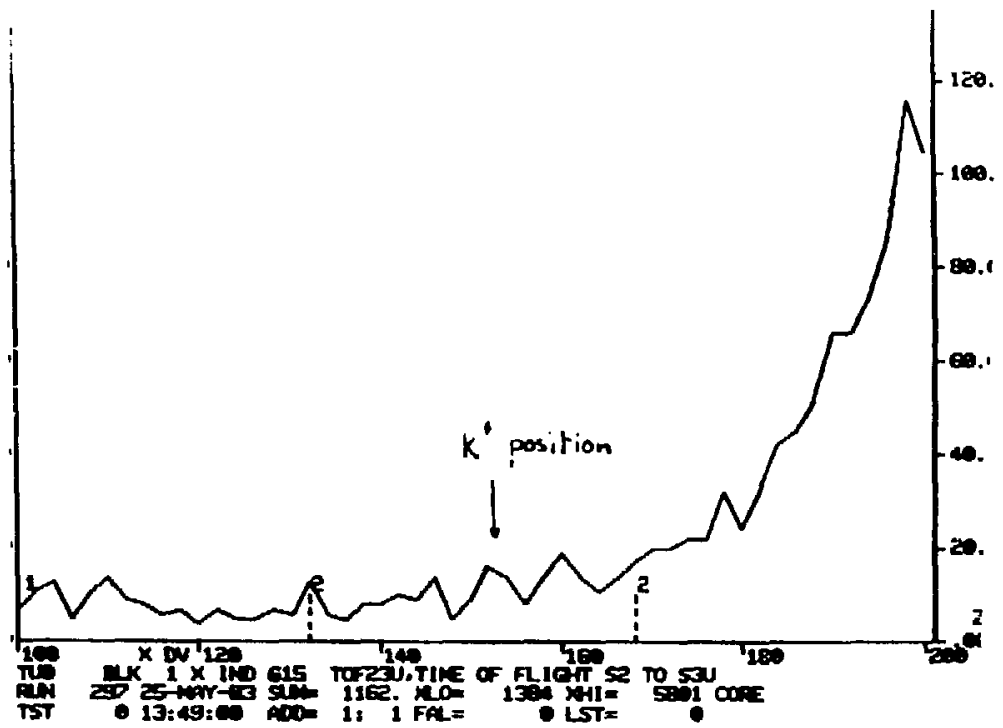

Figure 3.3.5.c. TOF spectrum in $\mathrm{Sp2}$, for events with no cuts applied. The kaon signal is not apparent.

Table $\underline{3.3 .5 . a}$

PMTs in

coincidence
( $\pi) \operatorname{AND}($ Cherenkov) $/(\pi)$

$=\pi$ efficiency
(K)AND (Cherenkov) /(K)

$=K$ efficiency

$\begin{array}{lll}N>1 & .998 & .630 \\ N>2 & .997 & .298 \\ N>3 & .995 & .122 \\ N>4 & .994 & .046 \\ N>5 & .989 & .012 \\ N>6 & .949 & .004 \\ N>7 & .684 & .002\end{array}$


Cherenkov detector at the rear of Sp2 identifies a particle with less mass than a kaon, namely a $\pi^{+}, \mu^{+}$, or $\mathrm{e}^{+}$.

There are ways in which the information from this simple detector may have been misleading. A kaon decaying in a forward angle, near the Cherenkov detector produces a $\mu^{+}$or a $i^{+}$with sufficient momentum to trigger the detector. Delta rays, or knock-on electrons, also could have sufficient $\beta$ to produce a trigger. These two effects serve to reduce the number of valid $\left(\pi^{+}, \mathrm{K}^{+}\right)$triggers by vetoing genuine $\mathrm{K}^{+}$signals. In addition, inefficiency in the PMTs would increase the number of invalid $\left(\pi^{+}, \mathrm{K}^{+}\right)$triggers by not vetoing $\left(\pi^{+}, \mathrm{X}\right)$ events. The total efficiency of the Cherenkov detector was measured by varying the number of PMTs required in coincidence with the pion signal. in Sp2 (results are displayed in Table 3.3.5.a). By requiring simultaneous signals in at least 5 of 7 PHTs in the detector, pions were identifiable at the 99\% level, while kaons produced a signal about $1 \%$ of the time. This allowed a vast decrease in the fast electronics dead-time.

\subsubsection{Momentum calibration of $\mathrm{Sp} 1$ and $\mathrm{Sp} 2$}

Because the spectrometer magnets were to be operated at several different central momenta, it was necessary to obtain a calibration over a wide range of fields. Magnet currents were monitored and controlled through a computer system called DIBBUK maintained by the 
AGS staff. In addition, Hall-effect probes monitored most magnets in Sp1 and Sp2, allowing a more precise control of field settings. The calibration strategy involved measuring the central magnetic salds directly by the "floating-wire" technique, while noting the Hall-probe voltage, producing a table of momenta versus Hall-vol tage.

The basis of the floating-wire technique is conceptually simple: a current carrying wire under tension $T$ in a magnetic field will assume a shape and position identical to the trajectory of a charged particle of momentum $P$ moving in the field. The relation is expressed

$$
P=2.9388 \frac{T}{i},
$$

eq. 3.3.6.a

with $P$ in $\mathrm{MeV} / c, T$ in grams, and $i$ in amperes, for convenience in units. Figure 3.3.6.a shows the circuit diagram and principal equipment used in the measurement. The current in eq. 3.3.6.a arises from the voltage across resistor $R$, the sense of polarity chosen to correspond to a positively charged particle beam moving in the positive z-direction. The wire was BeCu alloy ( $2 \% \mathrm{Be}), 4 \mathrm{mils}$ in diameter, kept under tension by a mass of 50 grams, and for some measurements, 80 grams. Since the wire was deflected in the $x$-direction by the magnet, the pulley had to be attached to its support by a hinge oriented along the y-axis, with the pulley free 


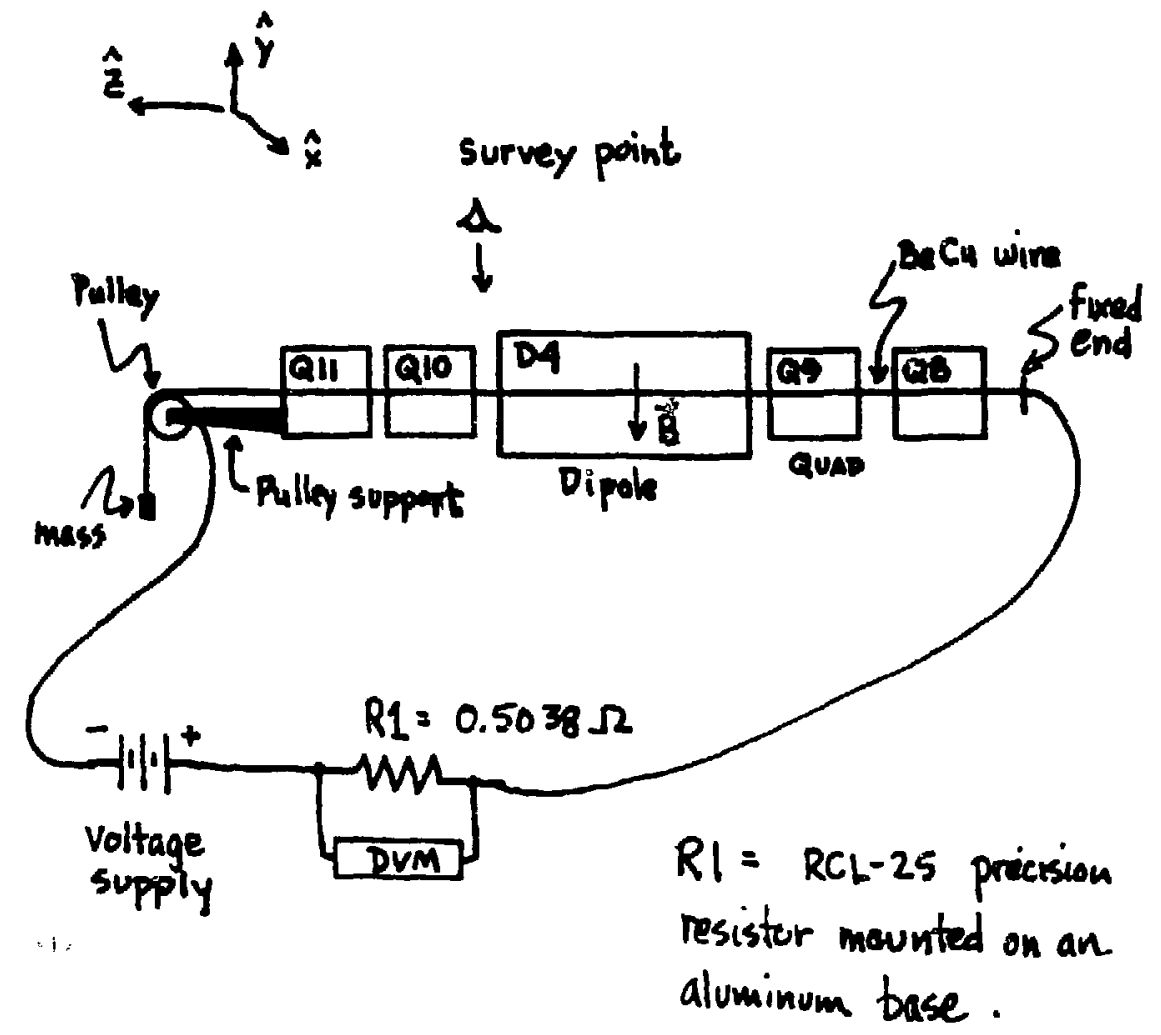

Figure 3.3.6.a. Sketch of circuit and equipment used to perform the floating-wire calibration of the Sp2 magnets. 
to pivot about the $y$-axis, as well as rotate in its usual motion about the $x$-axis, so as not to hinder the motion of the wire. An aluminum pulley and support were used, so for convenience, the cable from the voltage supply was connected directly to the pulley support. The fixed end of the wire was bound to a flange bolted to the face of magnet 08 , at the entrance of Sp2. The voltage connection here was made directly to the wire, completing the circuit. Mounting flanges for the pulley structure and stationary end were electrically isolated from the magnet bodies. All of these mechanical and electrical considerations were necessary to avoid interference with the wire, so that tension came only from the suspended mass.

A wide range of momenta were measured; from $500 \mathrm{MeV} / \mathrm{c}$ to the dipole maximum of roughly $1100 \mathrm{MeV} / \mathrm{c}$, in $50 \mathrm{MeV} / \mathrm{c}$ steps. Previously, BNL workers had obtained several values of the dipole magnetic field, and corresponding Hall-voltage, by the "flip-coil" method. These settings were used as a guide in finding the $50 \mathrm{MeV} / \mathrm{c}$ steps. The magnetic field is related to the central momentum by

$$
B=33.356 \frac{\mathrm{P}}{\mathrm{S}} \text {, }
$$

where $B$ is in kGauss, $P$ is in GeV/c, and $s=1.7056 \mathrm{~m}$, the length of the D4 magnet. Using equations 3.3.6.a and .b, voltage in the power supply was set to approximately produce a desired "momentum setting" 
in the floating wire. In practice, the voltage was varied slightly until the wire was centered in the dipole according to a transit survey alignment with scribe marks, defining the actual magnet momentum setting.

Appendix 3.3.4.a contains the data used in generating the polynomial curve fit $\left(X^{2} / N=2.4\right)$

$$
\begin{aligned}
V_{\text {hall }}= & \left(-2.65 \times 10^{-1}\right)+\left(2.26 \times 10^{-3}\right) * P+\left(-2.77 \times 10^{-6}\right) * P^{2} \\
& +\left(2.38 \times 10^{-9}\right) * P^{3}+\left(-6.59 \times 10^{-13}\right) \star P^{4} \text {, eq. 3.3.6.c }
\end{aligned}
$$

where $P$ is input in $\mathrm{HeV} / \mathrm{C}$, giving a functional form for the Hall-voltage, useful in setting the spectrometer during the experiment. The data in appendix 3.3.4.a are plotted in figure 3.3.6.b, graghically showing the smoothness of the measurement and combining both 50 and 80 gram points. Because of electrical power limitations at the time, only the dipole magnet D4 was operating while collecting these data points. However, since misalignment of a quadrupole magnet could introduce a "steering" component or a third-order optical aberration, it was necessary to perform the same measurement after the completion of the experiment, but with all four quadrupoles in Sp2 operating at field strengths used while collecting $\left(\pi^{+}, \mathrm{K}^{+}\right)$data. First, data were collected with only D4 operating for a comparison with earlier measurements. The D4 data were approximately reproduced, and when the quadrupoles were turned 


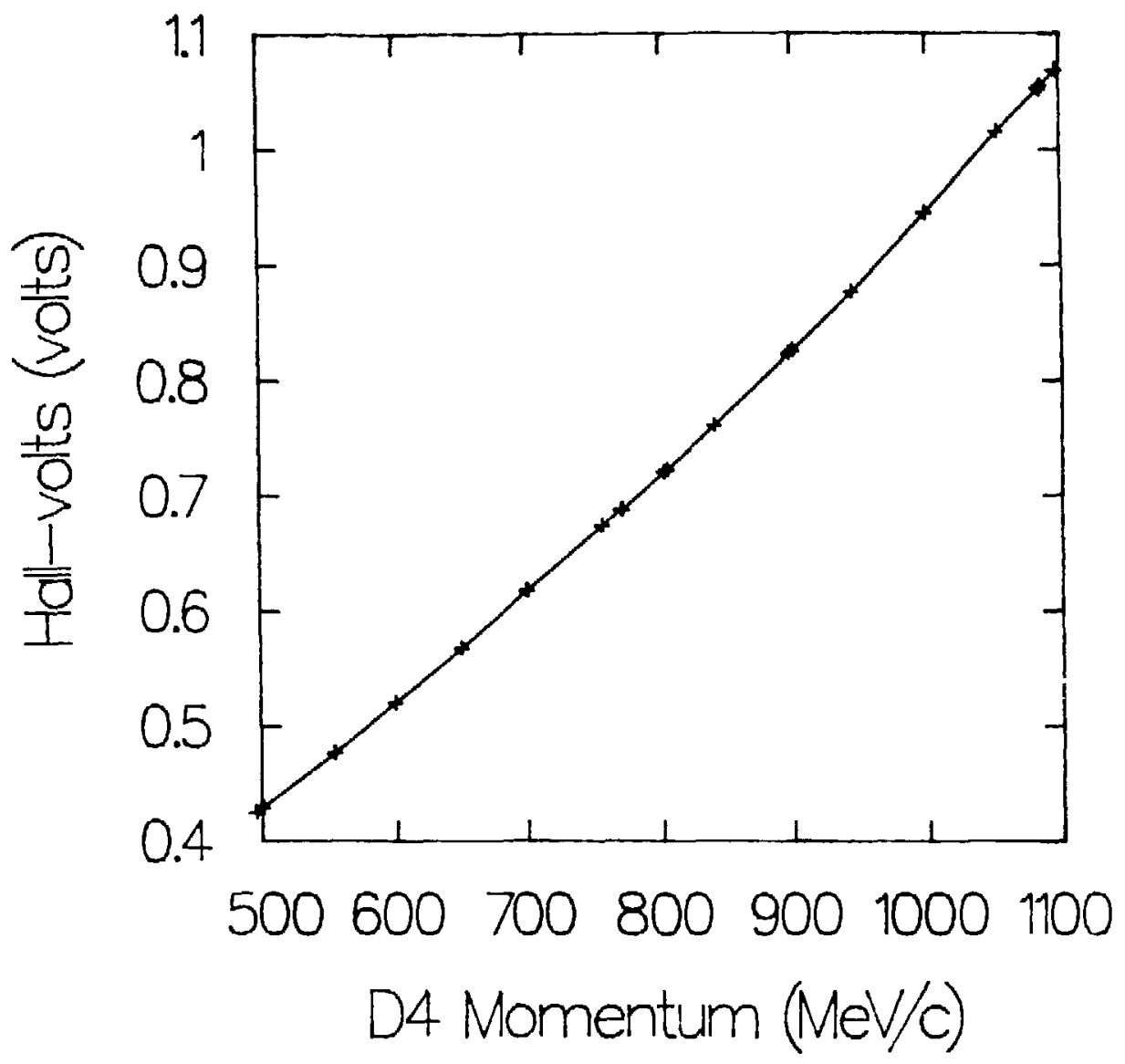

Figure 3.3.6.b. Data collected in the floating-wire procedure, giving a calibration of $\mathrm{Sp} 2$, in the momentum range 500 to 1100 $\mathrm{MeV} / \mathrm{c}$. A curve fitting this data is given by equation 3.3.6.a, useful in setting the spe-trometor at fields other than those directly measured. 
on, the wire vas not deflected, indicating there were no aberrations arising from these magnets. A polynomial fit to this second set of data (appendix 3.3.6.b, $x^{2} / \mathrm{N}=0.27$ ) gives

$$
\begin{aligned}
V_{\text {hall }}= & \left(-6.74 \times 10^{-1}\right)+\left(5.65 \times 10^{-3}\right) \star P+\left(-1.38 \times 10^{-5}\right) \star p^{2} \\
& +\left(1.96 \times 10^{-8}\right) \star \mathrm{P}^{3}+\left(-1.38 \times 10^{-11}\right) \star \mathrm{P}^{4} \\
& +\left(3.90 \times 10^{-15}\right) \star \mathrm{P}^{5},
\end{aligned}
$$

systematically giving momenta $-2 \mathrm{MeV} / \mathrm{c}$ higher than eq. 3.3.6. $\mathrm{c}$, for the same Hall-voltage. This corresponds to 0.23 grams of added tension in the wire, which could be attributable to: 1) increased friction in the pulley; or 2) soldering a lead to the floating wire to make the electrical circuit, instead of connecting to the pulley mount, as was previously done. A good electrical connection could not be made directly to the pulley mount during the lacter set of measurements.

While collecting the final set of Hall-voltage calibration data, a problem with the computer control of $D 4$ was discovered. Using the /LI command (to list magnet parameters on the CRT) on the DIBBUK system caused the D4 magnetic field to vary by $- \pm 1 \%$ for $2-3$ seconds. The problem had potentially serious implications for the da.a, since DIBBUK was usually placed in automatic monitoring mode during data collection, issuing the /LI command every 30 seconds. Magnet specialists from the AGS traced the problem to a faulty 
electronic controller which oscillated when interrogated by DIBBUK. The malfunctioning circuit was replaced, and D4 control was regained.

The effect of this variation of the 04 fleld on the spectrometer energy resolution is calculable as follows. Consider a central ray with $D 4$ at the expected setting; then $x_{f}=x_{r}=0$, $\theta_{\mathrm{f}}=\theta_{\mathrm{r}}=0$, and $\delta=0$. Now consider this same particle entering Sp2 after an excursion in the D4 field. Under this condition, $x_{f}=0, \theta_{f}=0$, but $x_{r}$ and $\theta_{r}$ are non-zero. When the /LI command on DIBBUK was used, the floating wire was observed to move about $1 / 2$ inch from the central position. This yields an approximately $\sim 0.5 \%$ spurious change in $\delta$. This result is smaller than the $\sim 1 \%$ variation observed in the Hall-voltage, but the frequency of oscillation of the field was probably shortar than the time constant for the oscillation modes of the wire, so that the wire did not reach full amplitude in response to the field. Finally, fluctuations were observed to last about 3 seconds. If the /LI command was issued every 30 seconds, this means $-10 \%$ of the good events were displaced by as much as $\delta=1 \%$ or $-4 \mathrm{MeV}$ in excitation energy . 


\subsection{Detectors}

Magnetic spectrometars provide a physical basis for measuring the momentum of a particle, depending on the phase space coordinates of the particle both before and after the spectrometer. Using pairs of position measuring detectors, these phase space coordinates are determinable.

Additionally, the mass of the particle must be known to calculate the total reiativistic energy. Mass species are identifiable by TOF differences over a known path length, for a given momentum. TOF differences are measurable by recording the time between pulses in scintillators at the front and rear of the spectrometer. Since they are simply required to measure the presence of a particle, timing scintillators are in the trigger counter category. A Cherenkov detector is also a trigger counter, but because the threshold and angle of Cherenkov light production depend on the $\beta$ of the particle, this detector can be used alone to distinguish between particle species, under certain conditions. The Cherenkov counter used in this experiment was discussed in section 3.3.5.

In this section, it is explained in trigger counters and position measuring detectors yield data used to calculate the momentum and energy of a particle which has passed through the spectrometer. 


\subsubsection{Trigger counters}

Particles passing through matter collide with electrons and nuclei. Collision cross sections with electrons are much higher than with nuclei because of the following classical considerations: the interaction is coulombic, and electrons fill a much greater volume, on the order of angstroms in diameter, while nucleons are confined to fermi diameters in their motion. The cross section for incident particles with charge is much higher than for neutrons, since neutrons can interact coulombically only via a magnetic moment. For incident charged particles more massive than electrons, the qualitative effects of collisions with electrons and nuclei are quite different. In collisions with electrons, a more massive particle loses energy without appreciable trajectory deflection. But in a nuclear collision, the more massive nucleus, with multiple charge, absorbs little energy, but significant alteration of the trajectory occurs because of the coulomb interaction. Therefore, charged particles lose energy passing through matter almost exclusively in collisions with electrons: this energy is converted by various processes into photons.

Scintillators are made of materials which have a high photon yield for these processes, and transmit photons well. A scintillator is optically coupled to a photomultiplier tube (PMT), so that the photon signal is amplified, giving an electronic trigger signal indicating the passage of a charged particle. 
The underlying processes for energy loss are the same in the Cherenkov effect, but the emitted photons constructively form a wavefront which is observed as a strong flash of light by a PMT. There is a threshold and angle associated with the wavefront; properties which can be exploited to increase the information derived from the detector.

\subsubsection{Time-of-flight scintillators}

The scintillators used were $1 / 4$ inch thick NE102 plastic, large enough in area to intercept all of the beam at their position. Since the beam covers a large area at the exit of Sp2, segmentation of both S3 and S4 into two sections, to maintain light gathering efficiency, was required. Also, each timing scintillator was monitored by two PMTs: one at each end fed into common coincidence circuitry to give a meantime signal for each pulse. This tecnnique cuts extraneous background, particularly in high contamination areas such as the mass slit cave, where S1 was located, producing a more stable timing trigger. For the position of the scintillators, refer to elements labelled $\mathrm{Sn}$ in figure 3.3.2.a.

Several essential event signals were constructed using information from S1, S2, and S3. Basically, they were of two types; S1-to-S2 TOF and S2-to-S3 TOF. Both a pion beam and kaon beam signal were derived from the $\mathrm{S} 1 \cdot \mathrm{S} 2$ coincidence. The $\mathrm{K} / \pi$ ratio was 
typically less than $1 / 1000$, so that the $K^{+}$component was not an important factor in the allocation of event processing priorities. A "loose" coincidence, giving the scattered event signal in Sp2, was formed by timing-in both pion and kaon signals over a la.ge energy range; the pions could later be rejected by the Cherenkov counter, or in software. This strategy facilitited quickly changing the event trigger between $\pi B \cdot \pi S, K B \cdot K S$, $\pi B \cdot K S$, etc., as required.

\subsubsection{Position measuring detectors}

Detecting the position of a particle is usually done in one coordinate: a strip of scintillator, or a wire aligned along a coordinate direction detects the passage of a particle. Since the $z$-position of the detector is known from an optical survey, this measurement gives 2 of 3 coordinates of the particle. Various strategies exist to economically measure the third coordinate; in this particular experiment, an identical drift chamber, rotated by $90^{\circ}$, was placed adjacent to the first detector.

\subsubsection{Scintillator hodoscope}

A scintillator hodoscope was employed between scintillator s1 and drift chamber DC1 in the mass slit cave. It consisted of 20 overlapping scintillators, each with dimensions $9 \mathrm{~mm} \times 1.25 " \times 125 "$ 
( $x$ by $y$ by $z$ ). The overlapping was done to give $3 \mathrm{~mm} x$-position resolution -- the elements were arranged in two rows, with $3 \mathrm{~mm}$ gaps between each element, with elements in each row centered over gaps in the other row. The detector covered 5 inches in the $x$-direction. Position information derived from $H 1$, in coincidence with DC1 position information, was used in software to eliminate spurious extra hits in $D C 1$, arising in the high back-ground rate environment of the mass slit cave. Small PMTs (RCA 8575) were used to amplify the raw signals.

\subsubsection{High-rate drift chambers (DCn)}

Drift chambers developed and constructed by the BNL members of the experimental collaboration were used for the first time, in the $\left(\pi^{+}, K^{+}\right)$experiment. Seven chambers, designated DC1-7 (figure 3.3.2.a), were used in the higher data-rate arsas of the mass slit cave and scattering target region.

Each chamber was composed of two planes of 24 wires each, separated by $4.5 \mathrm{~mm}$. A charged particie passing ihrough the first plane $(X)$ (figure 3.4.2.2.a) was sensed by ionization collecting on signal wires, shown as dots; the anode, or potential wires, are indicated by circles. In the second, adjacent wire plane $\left(X^{\prime}\right)$, the wires were offset by one drift distance from the first wire plane. This geometry improved the resolution of the detector, since 


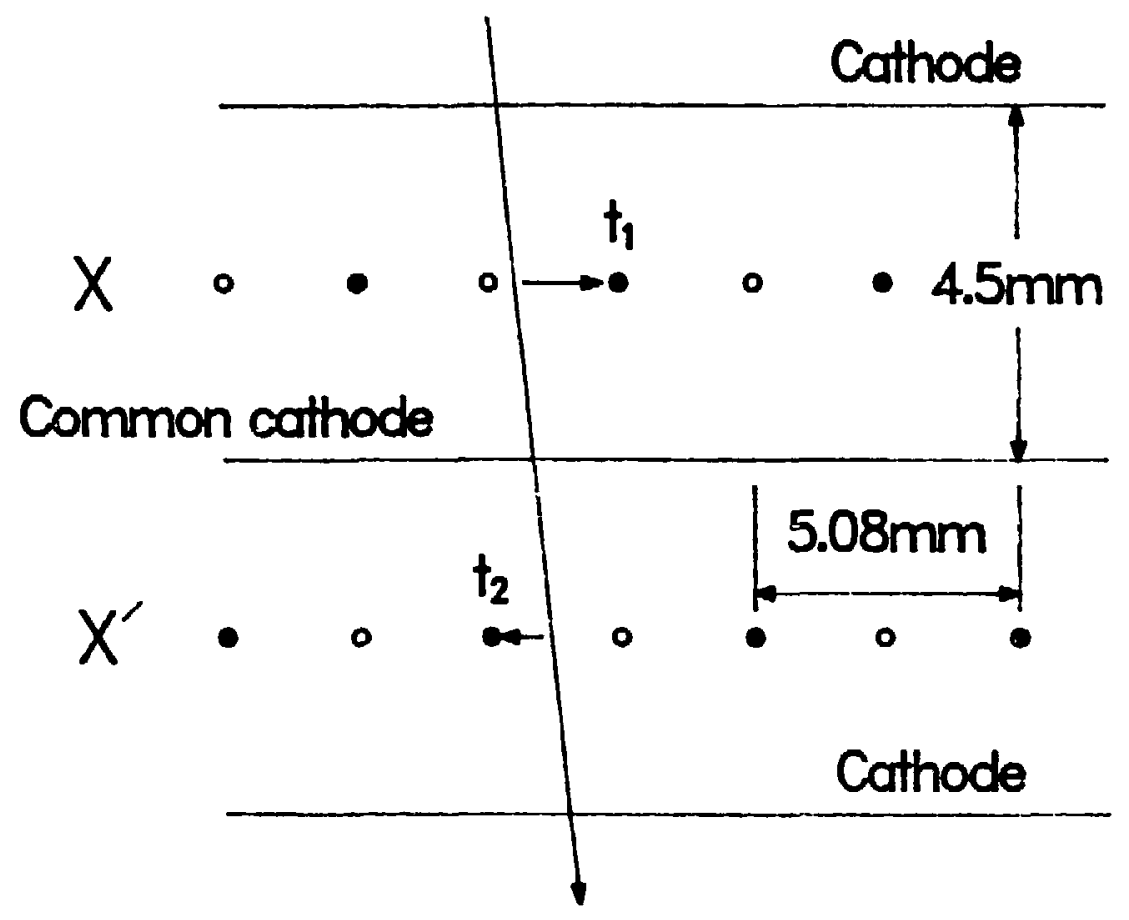

Figure 3.4.2.2.a. Diagram of the drift chamber, showing a cross sectional view of the two planes, $x$ and $x^{\prime}$, with a particle incident from the top of the page. Signal wires are shown as dots, potential wires as circles. For perpendicularly incident particles, $t_{1}+t_{2}=$ constant. The two planes are offset by one drift distance allowing the resclution of wire hit-side ambiguities. 
information from two planes was used to pinpoint the side of the wires on which the particle passed. A common-base pre-amplifier with emitter-follower formed a hybrid pre-amplifier which was compact and connected $2.5 \mathrm{~cm}$ from the acrive wires. The input capacitance was therefore low, minimizing both pre-amplifier noise contributions to the signals and discharge energy in the event of chamber arcing. The $2.2 \mathrm{~mm}$ cathode plane-to-wire spacing was possible because gas seals were made by coating the chamber with a mixture of $50 \%$ beeswax, $10 \%$ parafin, and $40 \%$ rosin, eliminating the need for thick o-ring seals. The chamber active area was $5 \mathrm{~cm}$ by $12 \mathrm{~cm}$, easily covering the typical 2 by $10 \mathrm{~cm}$ beam spot size. Signal wires were $10 \mu \mathrm{m} \mathrm{Au}-\mathrm{W}$ alloy, while potential wires were 50um $\mathrm{Cu}-\mathrm{Be}$ alloy. The distance between signal and potential wires (drift distance) was $2.54 \mathrm{~mm}$. The cathode planes (also serving as gas seals) were $25.4 \mu \mathrm{m}$ thick kapton sheets made conductive by a $2000 \AA$ evaporation layer of $\mathrm{Cu}$, held at -1250 volts. The anode wire vol tage was -1350 volts.

Initially, the chamber gas was a mixture of equal parts argon and ethane. But after only a few hundred hours of operation, chamber efficiency began to fall, ana so a small amount of methylal was introduced to the gas mixture to inhibit material deposition on the wires. Soon afterwards, two chambers failed when kapton windows, dissolved by the methylal, allowed air into the wire region, resulting in catastrophic electric discharges from the wire 
anodes to the plane cathodes. The kapton window failure was caused by a reaction of the methylal with type of kapton polycarbonate used in three of the drift chambers; the other chambers were free of this problem. However, it was discovered that in all of the chambers, carbonaceous crystal filaments had grown perpendicularly from the anode wires, following the electric field lines. These were most likely the original source of the falling chamber efficiency, allowing currents to develop from wire to plane which disrupted the potential patterns. The source of the carbon material was identified as a soft urethane glue used to bond the chamber frames. After this material was covered with a hard epoxy, the filament problem did not return. The chambers were cleaned, reactive kapton windows were replaced, and the chanbers re-installed. In subsequent experiments it was found further "aging" of filament growth on wires was inhibited by changing the chamber gas to $75 \%$ argon, $20 \%$ isobutane, and $5 \%$ methylal.

The signal processing was accomplished with electronics mostly built or modified by the BNL group [Pi 84]. From the pre-amplifiers mounted on the chamber chassis, the signal traveled 3 meters on 938 ribbon coaxial cable to a modified LeCroy 2735 aluplifier-discriminator card with 16 channels. The amplifiers on these circuit boards were grouped in four chip housings -- four amplifiers to a circuit package. The modifications by the BNL group mostly involved capacitative filtering to isolate all 16 circuits 
from one another, eliminating cross talk. Also, the cards were housed in NIM-standard modules and wrapped in copper foil to shield from stray electric fields. The amplifier introduced a \pm 4 ns variation in propagation time through the circuit, which was corrected in software, for each channel.

Figure 3.4.2.2.b shows a typical signal shape at the discriminator input, measured with a ${ }^{55}$ Fe $x$-ray source triggering the chamber. The potentials were somewhat lower than in practice, with the cathode plane at $-1000 \mathrm{~V}$ and $-1100 \mathrm{~V}$ on the anode wires.

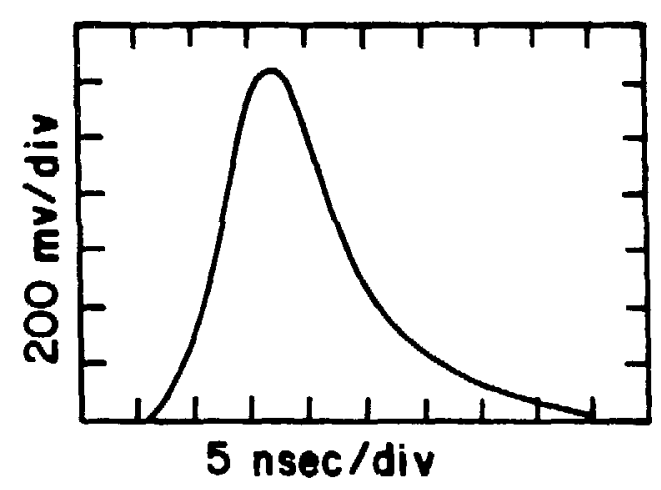

Figure 3.4.2.2.b. Typical signal shape from the hybrid pre-amplifiers going to the LeCroy 'iscriminators, measured with a ${ }^{55}$ Fe source triggering the chamber [Pi 84]. 
The LeCroy 2735 produced ECL-standard pulses, which were routed through $6 \mathrm{~m}$ of $100 \&$ twisted-pair cable to "homebrew" CAMAC multi-hit TDC units [Fa 81]. [ata from each wire were cyclically clocked at $250 \mathrm{MHz}$ into individual 128- or 256-channel TDC memories until an external stop signal (e.g., good $\pi \cdot K$ TOF) was supplied. In $\epsilon$.ct, a snapshot in time of all wire events was stored in the TDCs. The TDC required only one clock pulse (equal to $4 \mathrm{~ns}$ ) between the trailing edge of one signal and the leading edge of the next, so it was virtually free of dead time.

The performance of the chamber system can be seen in histograms of parameters measured with an incident pion beam of $5 \times 10^{6} \pi^{+} / 3$ at $\mathrm{P}_{\pi}=1050 \mathrm{MeV} / \mathrm{c}$. Figure 3.4.2.2. c shows the average output pulse width to be $38 \mathrm{~ns}$, giving the single-wire double-pulse resolution. Also shown is the histogram of ion drift time averaged over all (14 x $24=336$ ) wires in 14 drift planes. This figure gives an average reciprocal drift velocity of $18 \mathrm{~ns} / \mathrm{mm}$.

\subsubsection{Multi-wire proportional chambers (P3,P4)}

The chambers labelled "CMU" in figure 3.3.2.a were proportional chambers P3 and P4 constructed by the Carnegie-Mellon University group. The large active areas of $33.8 \times 15.2 \mathrm{~cm}$ for P3 and $33.8 \times 33.8 \mathrm{~cm}$ for $\mathrm{P} 4$ (horizontal by vertical) were required to cover the large beam phase-space at the rear of $\mathrm{Sp} 2$. Electronic 

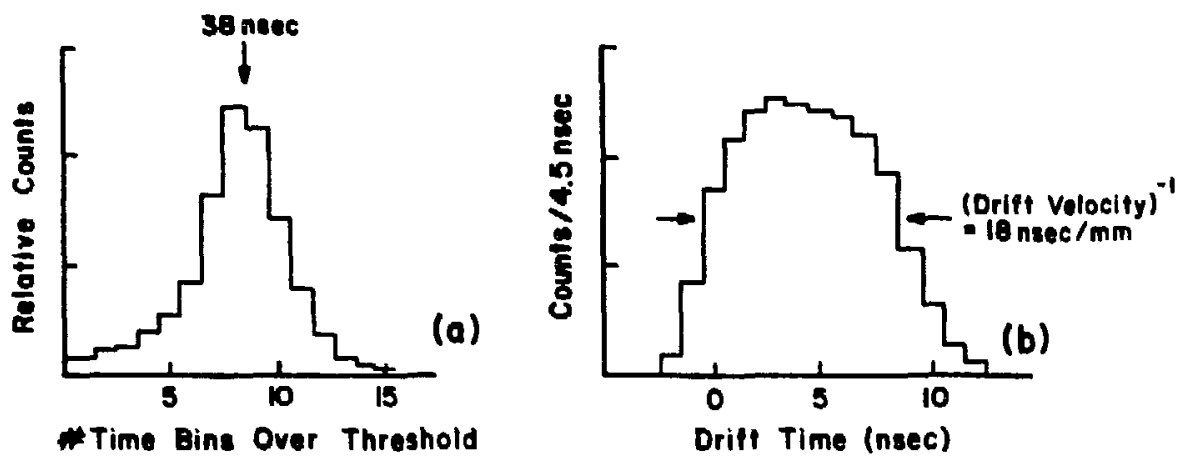

Figure 3.4.2.2.c. Average output pulse width from wires in the drift chamber. The width is $38 \mathrm{ns,} \mathrm{giving} \mathrm{the} \mathrm{effective} \mathrm{single} \mathrm{wire}$ double-pulse resolving time [Pi 84].

read-out was by PCOS amplifier cards mounted directly to the chamber frame. The sense wire spacing was $1.058 \mathrm{~mm}$. The chambers were replaced after the $\left(\pi^{+}, K^{+}\right)$experiment by drift chambers of the type discussed in the previous section. 


\subsection{Data acquisition}

The division of labor in acquiring data is naturally organized into two categories: hardware and software. Hardware includes all the electronic units which process and guide the flow of raw data from various detectors onto magnetic storage tapes. In principle, electronic hardware alone is sufficient, perform experiments and gather data. However, experimental complexity dictates using a computer to monitor and control magnetic field settings and proper detector function, for example. Also, quantities dealing with spectrometer optics and excitation energy spectra are calculable "on-line" during data gathering, providing procedural decision guidance. The reduction of vast volumes of data is possible only with a computer. Software has therefore become an indispensible experimental tool because it allows the experimenter to verify on-line the proper operation of an otherwise unmanageably complex experiment.

\subsubsection{Hardware}

Two types of hardware were used: 1) logic modules, hardwired to produce a signal only when specific event trigger conditions were present, and 2) programmable units, the micro-programmable branch driver (MBD) and the PDP-11/40 computer. The vast majority of incident pions did not produce a scattered kaon -- these " $\pi_{\text {beam }}$ 
only" events were rejected by fast logic, with only $\left(\pi^{+}, \mathrm{k}^{+}\right)$event triggers being passed along to the computer for processing and storage. The task of fast logic in general was: using TOF information, identify the small fraction of events of actual interest. When an event trigger was present, and the computer was not busy processing a previous event, the computer interface called computer automated acquisition and control (CAMAC) signalled the MBD to read all data for the event. The MBD then passed the data to the PDP-11 where it was stored on magnetic tape for later analysis.

\subsubsection{Trigger electronics}

The event trigger was based on TOF information. A time coincidence was formed between $S 1$ and $S 2$ for pions in Sp1, giving beam pion triggers, denoted $\pi B$. Similarly, a looser time coincidence between S2 and S3 was formed for pions and kaons in Sp2, called KS. Note that tightening the KS trigger in future experiments to exclude pions would improve trigger dead time. The coincidence $\pi \mathrm{B} \cdot \mathrm{KS}$ defined the event trigger. In addition, other triggers were formed for dianostic use: $K B$, $\pi S$, $n B \cdot \pi S$, and $K B \cdot K S$.

Figures 3.5.1.1.a, .b, and .c are schematic diagrams of the circuitry used to form $\pi B, \pi S, K B$, and $K S$ with signals from $S 1, S 2$, and S3. Abbreviaticns appearing in all three figures are defined in figure 3.5.1.1.a. riming scintillator pulses were read by two PMTs; 
one at each of two opposing ends. Timing circuits formed the "meantime" of the signal in the scintillator by merging the two PMT signals in a Lecroy 624 meantimer. A signal in S2 defined the start of timing. At the exit of $\mathrm{Sp} 2, \mathrm{~S} 3$ covered a large space, requiring the use of two large paddles, S3U (upper) and S3L (lower), which were treated as logically distinct timers.

When a $\pi B \cdot K S$ signal arose, it would be vetoed if a signal was present in the liquid nitrogen filled Cerenkov threshold counter, since such a signal would come from a pion, not a kaon. In figure 3.5.1.1.d, the circuitry processing the Cerenkov counter information is shown. The core of this circuit is the "multiplicity logic unit", which gives output according to a preset level of "N" input coincidences. In the present experiment, $\mathrm{N}=4$, so that the $\mathrm{kS}$ signal was vetoed only if 5,6 , or 7 of the PMTs monitoring the liquid nitrogen chamber simultaneously produced a signal. Additionally, the same signal level was required in coincidence with the $\pi S$ definition.

\subsubsection{CAMAC, MBD, and PDP-11/40}

The final steps in data acquisition occur when the trigger electronics signal the presence of an event through the LAMPF trigger module (LTM): data reading by CAMAC modules is halted, and all data is read by the MBD. When the computer is ready, it reads 


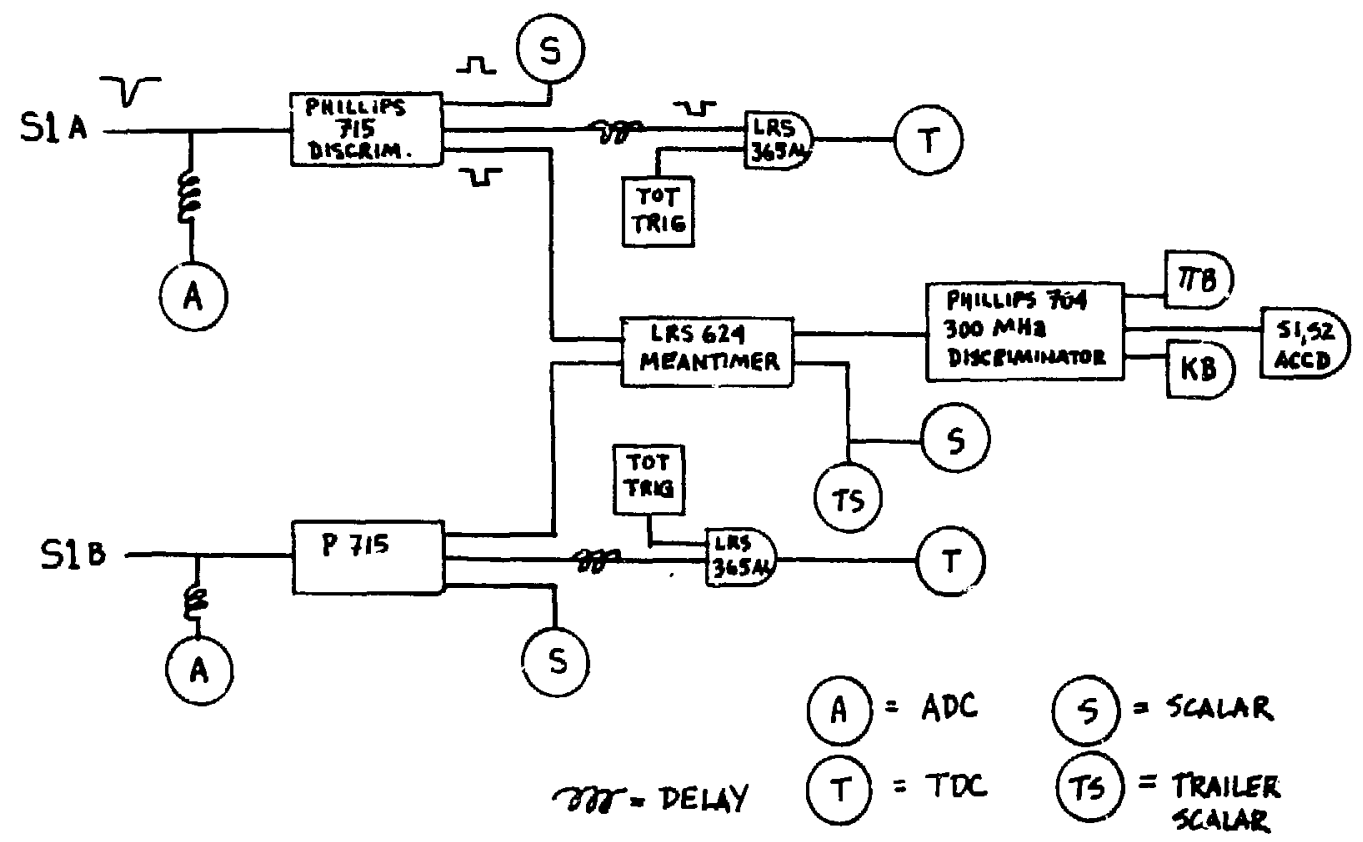

Fig're 3.5.1.1.a. Schematic diagram of circuit used to process raw data from timing scintillator $\mathrm{S} 1$. 


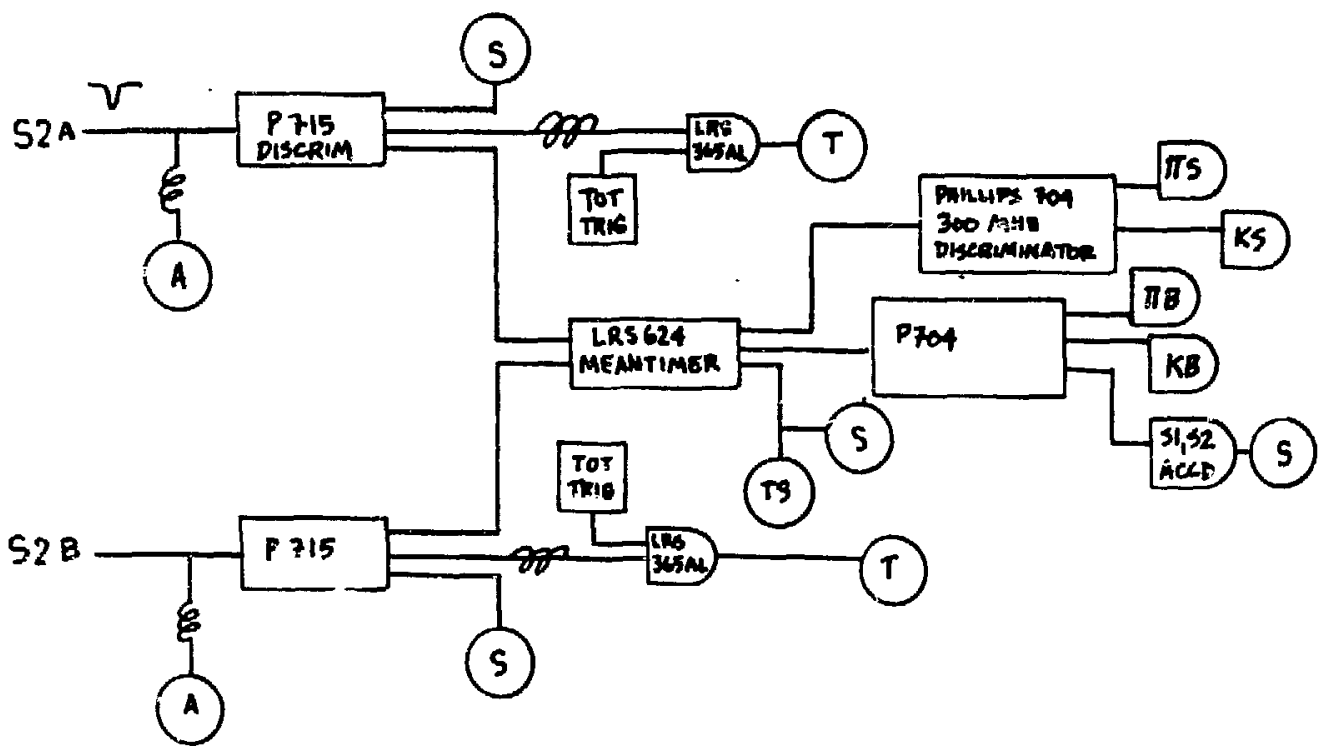

Tigure 3.5.1.1.b. Schematic diagram of circuit used to process raw pulses from timing scintillator 52 . This signal defined the start of Liming, and was used to define both "scattered" and "beam" events. 


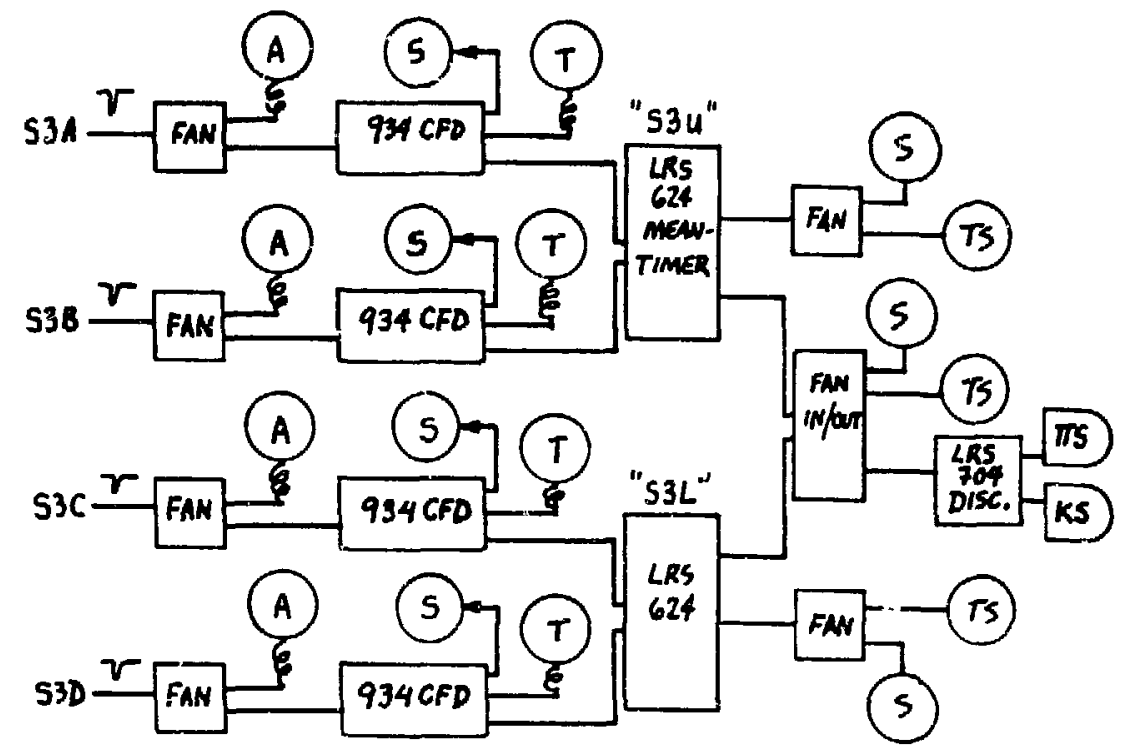

Figure 3.5.1.1.c. Schematic diagram of circuit used to process raw data from the two-sectio: scintillator S3. 


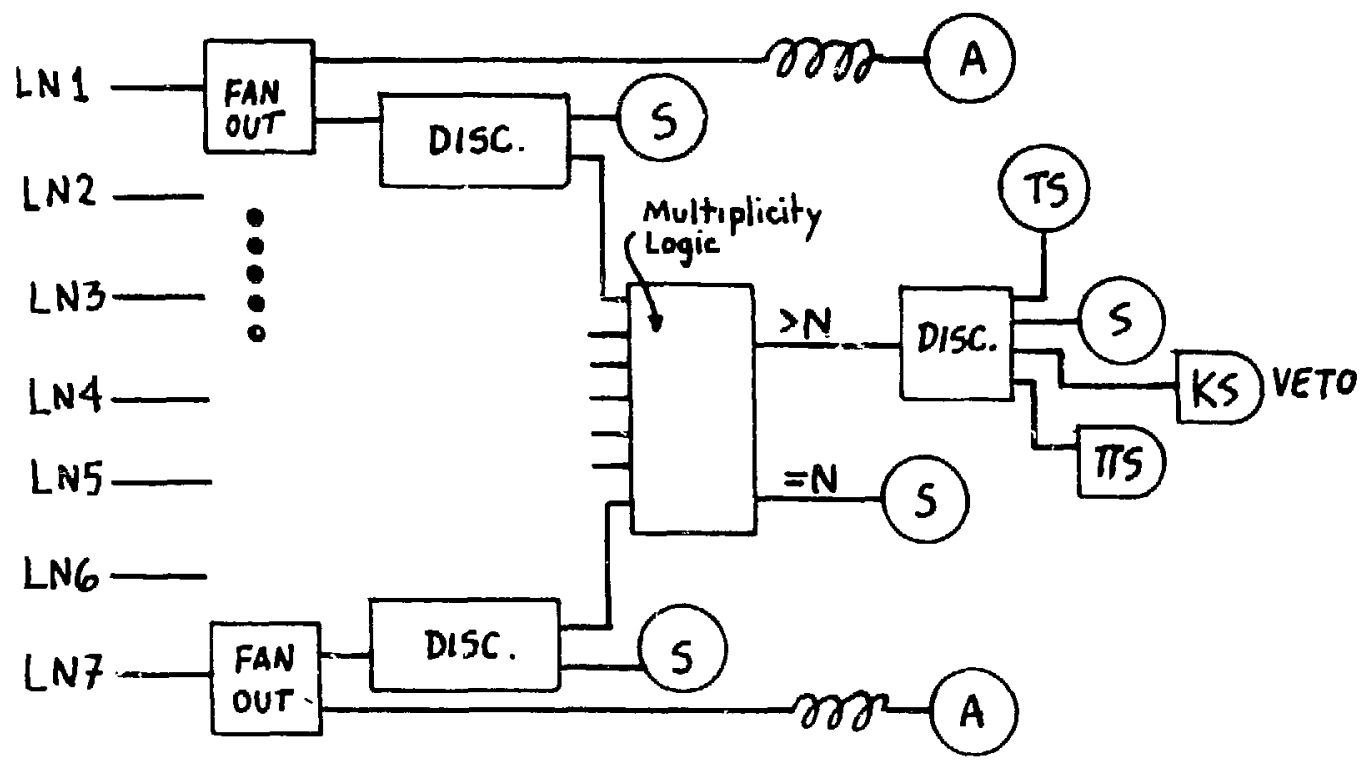

Figure 3.5.1.1.d. Schematic diagram of the circuitry used to process raw data from ti= PMTs in the liquid nitrogen filied threshold Cerenkov counter. The multiplicity logic unit was used to define the number of PMT pulses required in a coincidence to tag a pion event. 
the MBD buffers, storing the event on tape for later analysis, then passes an enabling signal back to CAMAC when it is finished, so the entire process can be repeated. As time allows, physical quantities like scattering angle and missing mass are calculated and stored in histograms.

CAMAC is a derined standard electronic system providing an interface between electronic modules and a computer. Scalars, TDCs, and ADCs reside in the CAMAC crates. An event trigger, in coincidence with a "run gate" (figure 3.5.1.2.a) signaling computer-not-busy, causes scalar inhibit and trigger hold-off signals to be issued. All data in the CAMAC scalar, TDC, and ADC registers are then frozen. In principle, the computer could now read this data directly over the CAMAC branch highway, using FORTRAN callable FCNA routines which operate on specific crates, slots, and channels in CAMAC. But an MBD provides a more efficient way of reading this data. The $M B D$ is a $4 \mathrm{~K}$ micro-computer with eight partitions capable of running micro-code on the command of the PDP-11 or a CAMAC look-at-me (LAM) signal. The MBD is alerted to the presence of an event by a signal in input EV6 of the LTM issuing a LAM. This causes the MBD to execute the Event 7 micro-code which reads all event data from CAMAC modules into an MBD data buffer. Running micro-code for Event 4 trarsiers the contents of the data buffer to the PDP-11. 


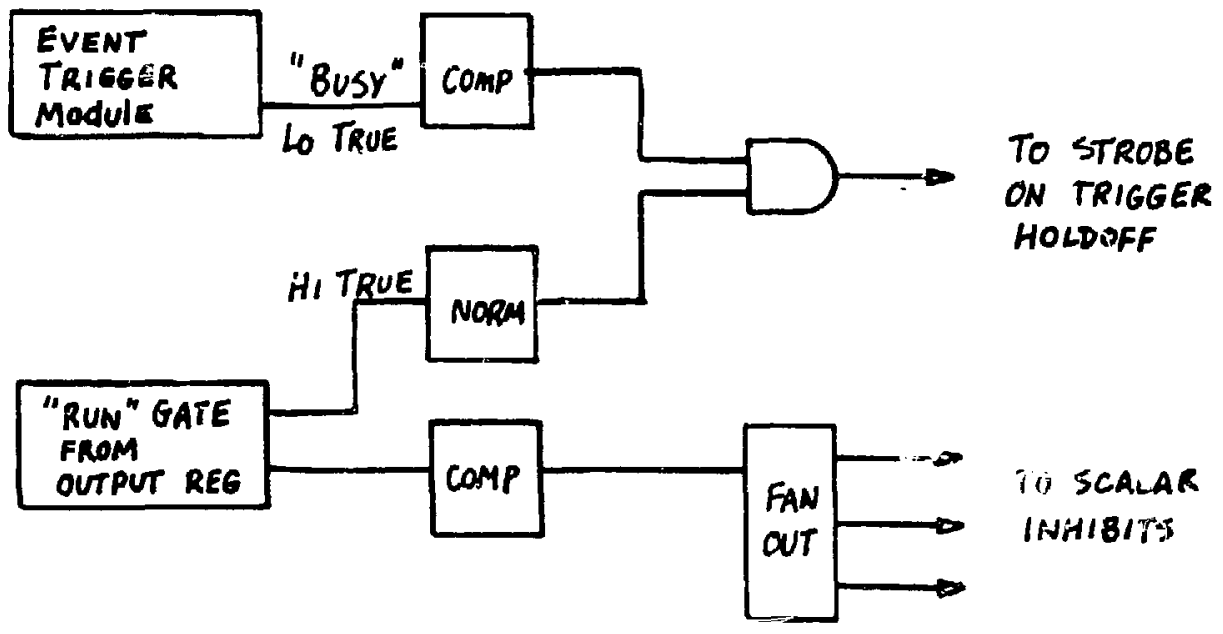

Figure 3.5.1.2.a. Schematic of circuitry used to define the "run gate" for collection of data. 


\subsubsection{Sortware}

The 0-system software is scribed in documents available from the LAMPF data analysis center. The discussion here includes a brief overview, along with a presentation of specific codes used for this experiment. The replyy-reduction-analysis program was nearly identical to the one used for data acquisition.

The main driver program, known as the analyzer, was named PROC7X. Event data buffers were allocated as arrays within the analyzer task. When the MBD completed a data transfer to this buffer, the PDP-11 wrote data to tape along with header information of date, time, etc. The analyzer recognized three different event modes: "must puocess", "mav process", and "no process". During $\left(\pi^{+}, \mathrm{K}^{+}\right)$data collection, the "may process" mode was used, meaning the computer was allowed to reduce data whenever it was not busy writing to tape, or reading an event. Both raw data and calculated quantities were then distribı' to various histogram files.

\subsubsection{The analyzer}

An snalyzer is a driver program that calls subroutines to calculate software corrections to raw data in addition to final kinematic quantities. The flow chart in figure 3.5.2.1.a gives the order of calculation -- there are no loops; only one pass per event was taken. 


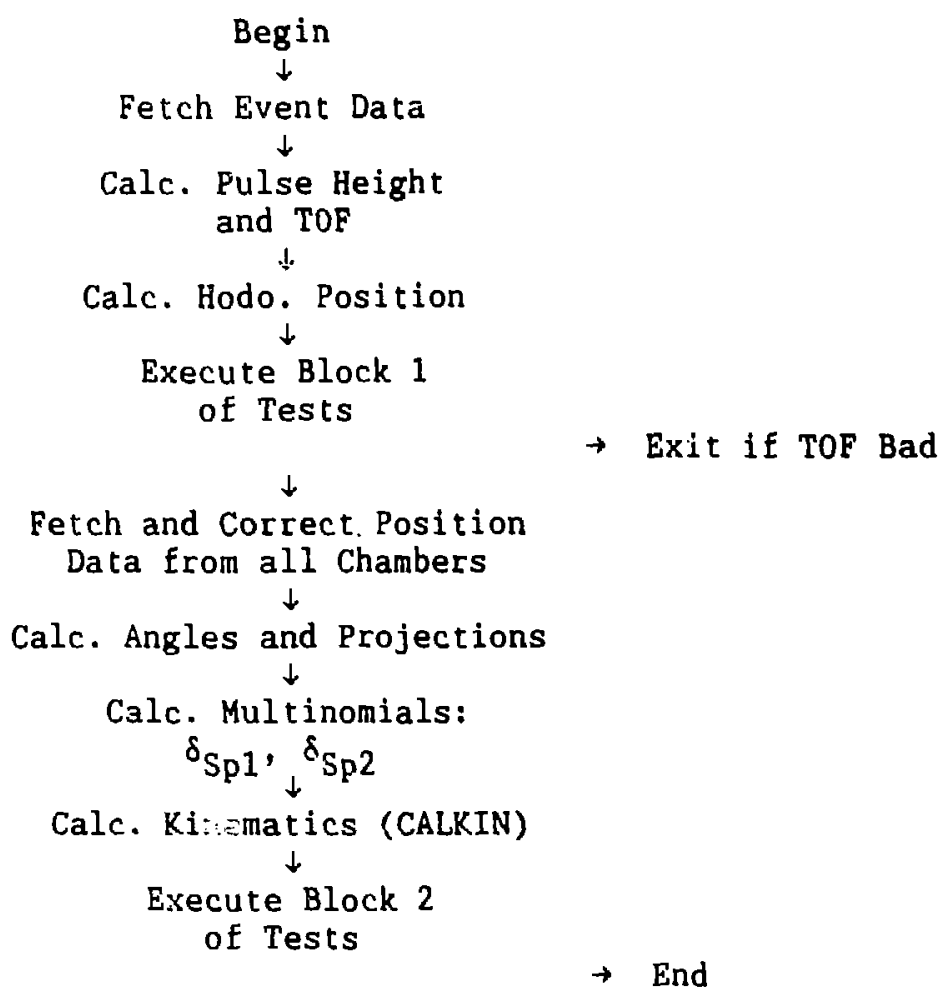

Figure 3.5.2.1.a. Flowchart fo" the one-pass Analyzer, PROC7X.FTN.

First, raw event data from the buffer was distributed to arrays accessible mnemonically. All relevant pulse height and TOF quantities were then caicuiated. Hodoscope information was converted to $x$-position. A wide TOF cut for kaons was imposed in BLOCK 1 of data wor' testing (described in the next section); 
further calculation was aborted if the particle did not pass the TOF cut.

Next, processing of position information began by correcting position data for all chambers according to measured individual drift wire characteristics and TDC calibrations. Knowing positions, angles and position projections were calculable. These were straightforward calculations: using z-positions for chambers, and measured $x-$ and $y$-positions, $\Theta$ ( $x$-angle), and $\phi$ (y-angle) were computed at the exit of $\mathrm{Sp} 1$, and at the entrance and exit of $\mathrm{Sp} 2$. In addition, $x$-position at tha scattering target was projected from position and angle information. With all positions and angles computed, $\delta_{\mathrm{Sp} 1}$ and $\delta_{\mathrm{Sp} 2}$ were calculable using TRANSPORT-like coefficients contained in the special parameter file POL.DAT. The advantage of $\delta$-polynomial coefficients residing in a called file is flexibility; they can be changed easily during spectrometer optics studies. Using $\delta_{\mathrm{Sp} 1} \delta_{\mathrm{Sp} 2}$, reaction participant masses, energy losses, and spectrometer central momenta values, the reaction missing mass was calculated with the kinematics routine, CALKIN. This routine was identical to the one used at HRS and EPICS at LAMPF, except for an exchange of $\theta$ and $\phi$ to account for the orientation of $\mathrm{Sp} i$ and $\mathrm{Sp} 2$ being rotated by $90^{\circ}$ in the scattering plane (these spectrometers lay on their "sides" compared to HRS). Finally, BLOCK 2 of the tests was executed, in which good hypernuclear events were positively identified. 


\subsubsection{Data-word testing}

Several hundred data-words, residing in real and integer arrays, were separately testable. These words included chamber hit information, TOF, and all calculated positions, projections, and angles. The tesis which could be applied to the data words included GATE(True if the data-word value is within specified bounds), EOUAL (True if value is equal to a specified constant), and logical tests on two or more data-words: AND, OR, EOR (Exclusive OR), e:s. Tests were combined logically to define $\left(\pi^{+}, \mathrm{K}^{+}\right)$event validity out of the set of trigger events passed to the computer.

The tests, or cuts, applied to the events passed to BLOCK 2 of PIKNEW.TST (Appendix 3.5.2.2.a). were: 1) one possible hit in DC1 AND DC2 AND DC3; 2) one possible hit in DC5 OR DC7; 3) exactly one hit in "CMU" multi-wire proportional chambers $\mathrm{P3}$ and P4; 4) proper vertex projection (insuring the scattering occurred in the target); 5) kaon TOF in $S 2$ to $S 3 ; 6) \pm 27 \mathrm{mr}$ window on $\theta$ at the exit of Sp1; 7) $-1.3 \mathrm{~cm}$ to $+1.6 \mathrm{~cm}$ window on $y$-direction at the exit of Sp2; and 8) $\pm 5 \mathrm{mr}$ window on $\theta$ projected to the entrance of $\mathrm{Sp} 2$ with rear $\mathrm{Sp} 2$ information, minus $\theta$ measured at the entrance of Sp2 -- called "theta check". Good $\left(\pi^{+}, \mathrm{K}^{+}\right)$events passed a test requiring passage of an AND of all eight cuts. 


\subsection{The scattering target}

The lifetime of the $\Lambda$ particle bound to the nucleus is about 0.2 ns [Gr 85], effectively in coincidence with the $\left(\pi^{+}, \mathrm{K}^{+}\right)$event trigger. A large amount of energy is released in the $\Lambda$ decay, fragmenting the nucleus and ejecting energetic nucleons, or a nucleon and a pion. Observation of this decay can be exploited to provide a confirmation, in combination with the normal event trigger, that hypernuclear form. ion occurred. The simplest method is to use a plastic scintillator with PMT readout as a scattering target. This is known as a "live target", since in addition to serving as the scatterer, it detects the energy loss of products of the $\Lambda$ decay.

The beam spot size at the pivot position between Sp1 and Sp2 vas about $8 \mathrm{~cm} \times 2 \mathrm{~cm}$ (x-direction by $y$-direction), defining the minimum requíred target dimensions. An NE102 scintillator measuring $1 " \times 2 " x 4 "$ ( $z$ by $y$ by $x$ ) was used as the scattering target. It was attached to a PMT by optical glue and shielded from exterior light in the usual way by a wrapping of aluminum foil and black electrical tape:

Scintillator plastics (such as NE102) are made of polystyrene, a large organic molecule with an atomic ratio of $\mathrm{H} / \mathrm{C}=1.10[\mathrm{Ba} 80]$. Using this information, and assuming additive densities, the density for the ${ }^{12} \mathrm{C}$ constituent in the target may be extracted: 


$$
\rho_{C}=\rho_{\text {Scint }}-\rho_{H}=0.940 \mathrm{~g} / \mathrm{cm}^{3} \text {, }
$$

eq. 3.6.a

where $\rho_{\text {scint }}=1.032 \mathrm{~g} / \mathrm{cm}^{3}$, and $\rho_{H}=0.092 \mathrm{~g} / \mathrm{cm}^{3}$. Using this density, the effestive scattering center area was

$$
\frac{A}{N_{0} \rho d}=8.39 \times 10^{-24} \mathrm{~cm}^{2} \text {, }
$$

where $\mathrm{N}_{0}$ is Avogadro's number, $A$ is $12 \mathrm{~g}$ for the atomic weight of ${ }^{12} \mathrm{c}$, and $\mathrm{d}$ is $2.54 \mathrm{~cm}$ for the thickness of the target. Note that the presence of hydrogen in the target was relevant only as a consideration in this density calculation, since the $\left(\pi^{+}, \mathrm{K}^{+}\right)$ reaction cannot proceed on the hydrogen nucleus.

Energy lost by charged particles reaches a minimum at $\beta=0.96$, almost independent of the medium. However, for $\mathrm{P}_{\mathrm{K}}=716 \mathrm{MeV} / \mathrm{C}$, $\beta_{K}=0.823$, and for $P_{11}=1054 \mathrm{MeV} / \mathrm{c}, \beta_{\pi}=0.991$, so that one would expect tha energy loss for these particles in the target to vary from the "minimum ionizing" condition. For relativistic particles, the energy loss is given by

$$
\frac{d E}{d x}=\frac{D \rho_{m} z_{m}}{A_{m}}\left(\frac{Z_{i}}{\beta}\right)^{2}\left[\ln \left(\frac{2 m_{e} r^{2} \beta^{2}}{I}\right)-\beta^{2}\right],
$$
eq. $3.6 \cdot c$

where the subscripts $m$ and $i$ refer to the medium and the incoming particle, respectively. Also, $D=4 \pi \mathrm{N}_{0} \mathrm{r}_{\mathrm{e}}^{2} \mathrm{~m}_{\mathrm{e}}=0.307 \mathrm{MeV} \cdot \mathrm{cm}^{2} / \mathrm{g}$ and 
$I=16\left(z_{m}\right)^{0.9} \mathrm{eV}$ for $z_{m}>1$, to account for binding of atomic electrons. For $z_{m}=1$, the ionization energy is $I=13.6 \mathrm{eV}$. In a target such as NE102, which is a chemical mixture, the energy loss may be approximated by

$$
\frac{d E}{d x}=\sum_{n=1}^{N}\left(\frac{d E}{d x}\right)_{n},
$$

where $(d E / d x)_{n}$ corresponds to the $n^{\text {th }}$ chemical constituent, using $\rho_{m}^{n}, z_{m}^{n}$, etc., as the "partial" density, charge, etc., of the medium. Using eqs. 3.6.c and 3.6.d gives $\mathrm{dE} / \mathrm{dx}=2.50 \mathrm{MeV} / \mathrm{cm}$ for $\mathrm{K}^{+}$at $P_{K}=716 \mathrm{MeV} / \mathrm{c}$, and $\mathrm{dE} / \mathrm{dx}=2.25 \mathrm{MeV} / \mathrm{cm}$ for $\pi^{+}$at $P_{\pi}=1054 \mathrm{MeV} / \mathrm{c}$. These numbers reflect the mean energy loss of an energy loss distribution function. In calculating the missing mass, these numbers were used, together with the simplifying assumption that the "'pernuclear interaction always occurred at the middle of the 1" thick target. For $\pi^{+}$, this constitute $E_{\text {loss }}=2.86 \mathrm{MeV}$, and for $\mathrm{K}^{+}, \mathrm{E}_{\text {loss }}=3.17 \mathrm{MeV}$.

The signal from the live target was fed into both a TDC and an ADC. The voltage on the PMT was set low, at $\approx 1300 \mathrm{~V}$, since only large pulses were of interest.

A live target is useful only if it gives information positively correlated with an event. In the present experiment, the live target yielded ambiguous data on the formation of hypernuclei. This can be seen by histogramming the tio of: (good events lacking a 


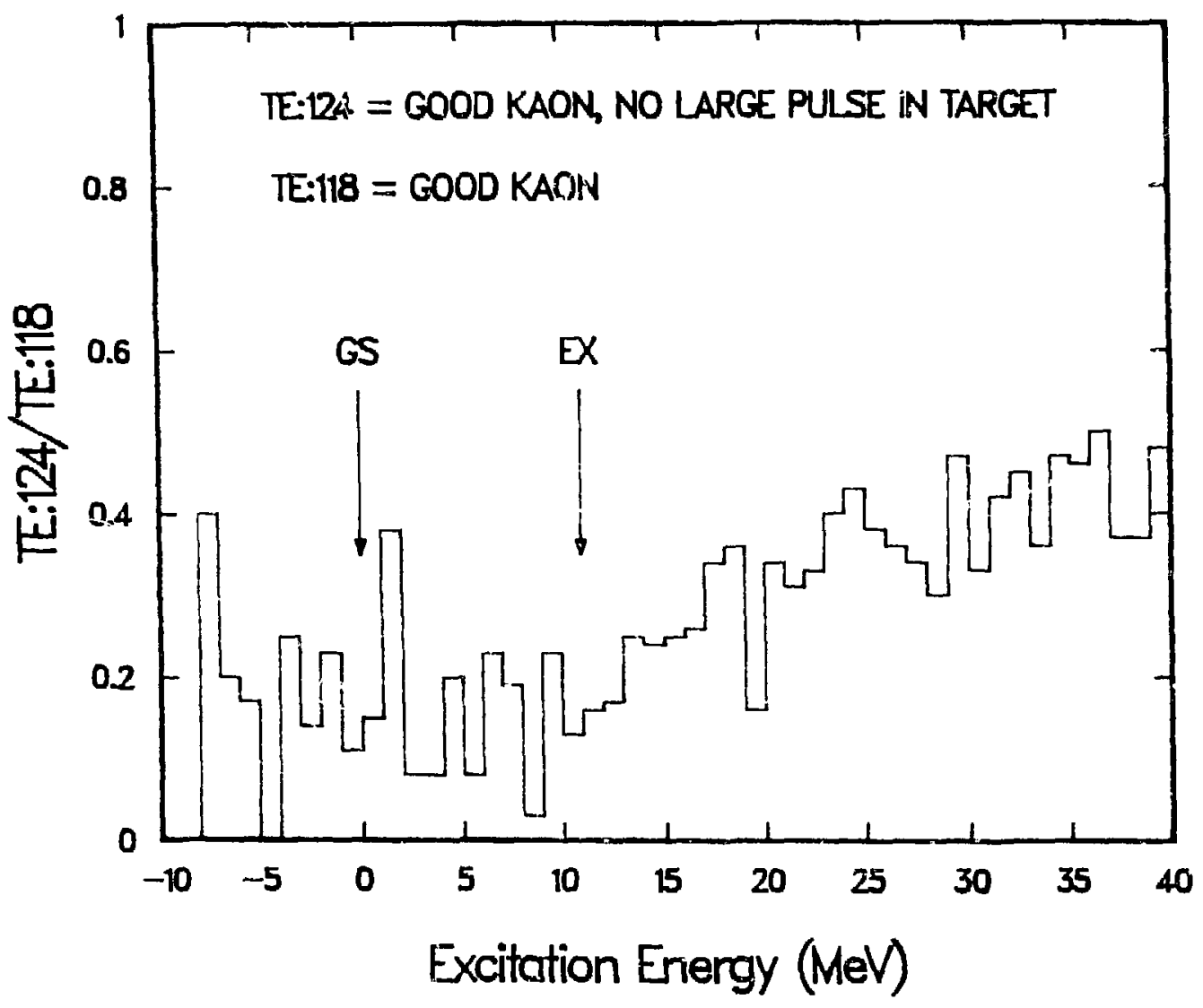

Figure 3.6.a. Fraction of good events which were not coincident with a large pulse height in the live target. The positions of two spectral peaks observed in other histograms, are indicated. The absence of counts in the peak region suggests the live target inay have been working as intended, although not decisively. 
large live target pulse height)/(good events), as a function of hypernuclear excitation energy. This histogran (figure 3.6.1) shows a flat distribution of good events for which there was no large pulse height. This implies that the live target pulse height software cut served only to reduce the effective hypernuclear event rate, and therefore introduced greater statistical counting error. In the spectra presented in section 4.1, the live target pulse height cut was not used. 
CHAPTER FOUR

\section{Experimental resuits}

The experimental results consist of three excitation energy spectra for the ${ }^{12} \mathrm{C}\left(\pi^{+}, \mathrm{K}^{+}\right){ }_{\Lambda}{ }^{12} \mathrm{C}$ reaction, collected at spectrometer settings of 5,10 , and 15 degrees. Prominent structures in the spectra are discussed in this chapter, along with the associated topic of spectrometer energy resolution. Errors connected with knowledge of peak strengths are presented, leading to a calculation of the differencial scattering cross sections for each spectrometer setting.

\subsection{Hypernuclear spectra}

Excitation energy $\left(E_{e x}\right)$ spectra recorded for $\Lambda^{12} \mathrm{C}$ for laburatory angles of 5.6, 10.3, and 15.2 degrees are shown in figure 4.1.a. These angles are the centroids of empirically determined kaon spectrometer accep inces corresponding to spectrometer angle settings of 5,10 , and 15 degrees. The determination of these angles is discussed later in this section. 


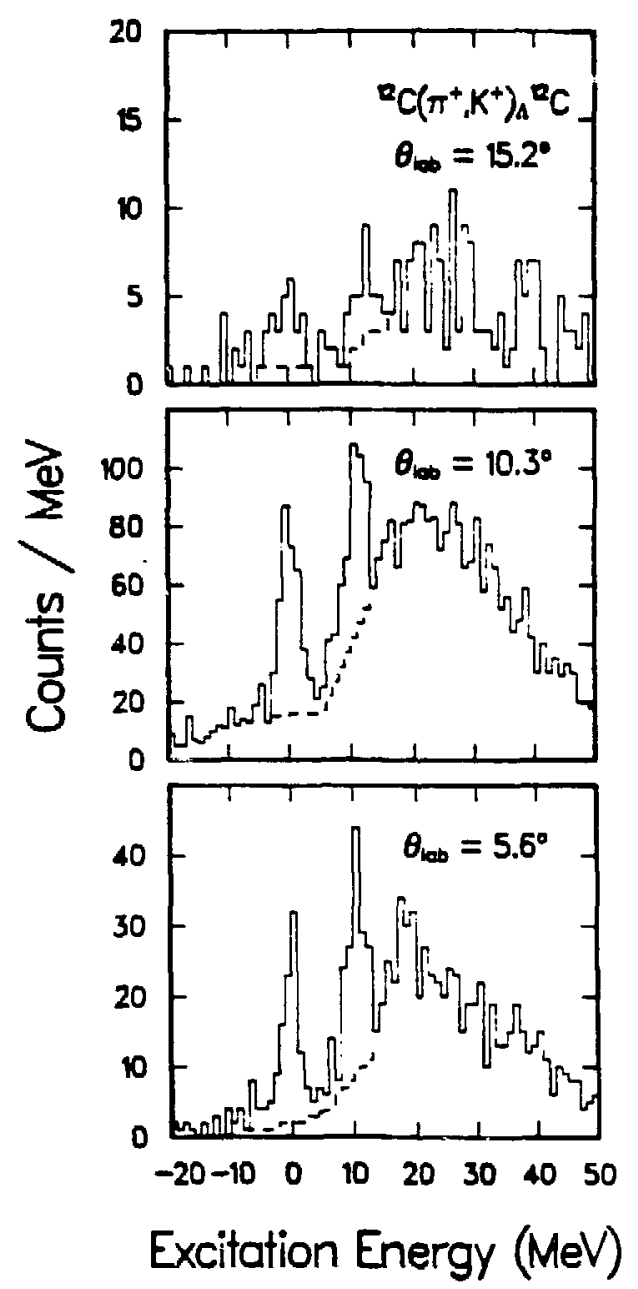

Figure 4.1.a. Excitation energy $\left(E_{e x}\right)$ spectra for $\Lambda^{12} \mathrm{C}$ excited in the $\left(\pi^{+}, \mathrm{K}^{+}\right)$reaction at laboratory angles of $5.6,10.3$, and 15.2 degrees. The incident pion momentum is $1054 \mathrm{MeV} / \mathrm{c}$. These spectra have been shifted $4 \mathrm{MeV}$ to the right as described in the text. 
Several features are common to all three spectra. Most important are the two peaks, at about $E_{e x}=0 \mathrm{MeV}$ and $E_{e x}=11 \mathrm{MeV}$. They appear with the same energy spacing reported in the previous studies [Ch 79] of ${ }^{12} \mathrm{C}\left(\mathrm{K}^{-}, \pi^{-}\right) \Lambda^{12} \mathrm{C}$, as expected. As shown, the spectra have been shifted $4 \mathrm{MeV}$ to the right (that is, the ground state originally appeared at $E_{e x}=-4 \mathrm{MeV}$ ). The sources for this error in the energy scale most likely arise from the rather large momentum difference of the two spectrometers of $-330 \mathrm{MeV}--$ this is discussed more fully in section 3.3.6, "Homentum calibration of Sp1 and $\mathrm{Sp} 2 "$. Because of the similarities in the energy level spacing with the ${ }^{12} \mathrm{C}\left(\mathrm{K}^{-}, \pi^{-}\right){ }_{\Lambda}^{12} \mathrm{C}$ case, it is reasonable to identify the peak at $\mathrm{O} \mathrm{MeV}$ as the ground state. The original, unshifted spectra data tables, from which the graphical spectra are generated, appear in appendix 4.1.a.

In the negative $E_{e x}$ region appear events arising from pole-face scattering, particle misidentification, and perhaps other extraneous contributions. This distribution is presumahly present throughout the full range of the spectrum. It is hoped that in future experiments, increasing the number of x-direction drift planes to three at the entrance of $\mathrm{Sp} 2$ will reduce the vertex projection ambiguities that allow these events to pass all the cuts. This is especially important for observation of states in ${ }^{28} \mathrm{Si}$ and ${ }^{40} \mathrm{Ca}$, for example, where the cross sections are expected to be in the $1 \mu \mathrm{b} / \mathrm{str}$ range, similar to the strength of the background signal seen here. 
Above $E_{e x}=11 \mathrm{MeV}$, in the unbound $\Lambda$ region, the broad distribution results from the quasi-free process $\pi^{+}+n \rightarrow \Lambda+k^{+}$. The limitation of the spectrometer acceptance causes the distribution to decrease in magnitude after $E_{e x}=20 \mathrm{MeV}$. An interesting feature in the $\theta_{\text {Lab }}=5.6^{\circ}$ spectrum is the possibility of a faint peak near $\mathrm{E}_{\mathrm{ex}} \simeq 1 \varepsilon \mathrm{eV}$. This possible state is discussed in section 5.1 along with shell podel configuration assignments for the other states.

Dashed lines under the two peaks represent an extrapolation from the background region to the continuum region for the purpose of background subtraction. These lines were chosen subjectively and do not contain any physics.

The centroid of the spectrometer acceptance is not the same as the relative angle formed by the centerlines of the two spectrometers. To understand this, imagine a spectrometer with acceptance \pm 2 degrees, and set with $i$ ts centerline at zero degrees with respect to the incident beam. In this case, the acceptance centroid differs from zero degrees for two reasons: 1) the $x$-direction centroid must have some value corresponding to $0^{\circ}<|\theta|<2^{\circ}$, depending on how the reaction cross section strength varies across the acceptance; and 2) in general, the particle has a non-zero scattering-angle. In the subroutine CALKIN of PROC7X, THTSCT (scattering angle relative to the spectrometer lab setting) 
is computed by transforming the actual scattering angle (a function of both $\theta$ and $\phi$ ) to an angle $\theta$ in the $x-z$ plane.

The effective centroid of the spectrometer lab angle is equivalent to the centroid of THTSCT. But using the raw THTSCT data to compute the centroid would be incorrect. In general, the cross section intensity varies across the spectroneter acceptance, and THTSCT must be corrected for this in order to calculate an accurate centroid. The raw THTSCT distribution was subsequently corrected using the theoretical cross section distribution in calculating the centroid. The THTSCT distribution at $\theta_{\text {Lab }}\left(10^{\circ}\right)$ (not yet corrected for cross section fall-off) is shown in figure 4.1.b. Events in this histogram pass all of the regular $\left(\pi^{+}, \mathrm{K}^{+}\right)$requirements-- and in addition, fall into the ground state peak region, and within a solid angle defined in software by $-27 \mathrm{mr}<\theta<+27 \mathrm{mr},-80 \mathrm{mr}<\phi<+80$ mr. Using this method, the THTSCT centroids for the three lab settings are $\theta_{\mathrm{Lab}}\left(5^{\circ}\right)=5.6^{\circ} \pm 0.9^{\circ}, \quad \theta_{\mathrm{Lab}}\left(10^{\circ}\right)=10.3^{\circ} \pm 0.9^{\circ}$, and $\theta_{\text {Lab }}\left(15^{\circ}\right)=15.2^{\circ} \pm 0.6^{\circ}$, whore the "uncertainty" limits refer to an angular interval required to encompass $67 \%$ of the events in the histogram. It should be noted that this calculation is statistically poor, since only 69 events were used at 5 degrees, 238 events at 10 degrees, and 20 events at 15 degrees. 


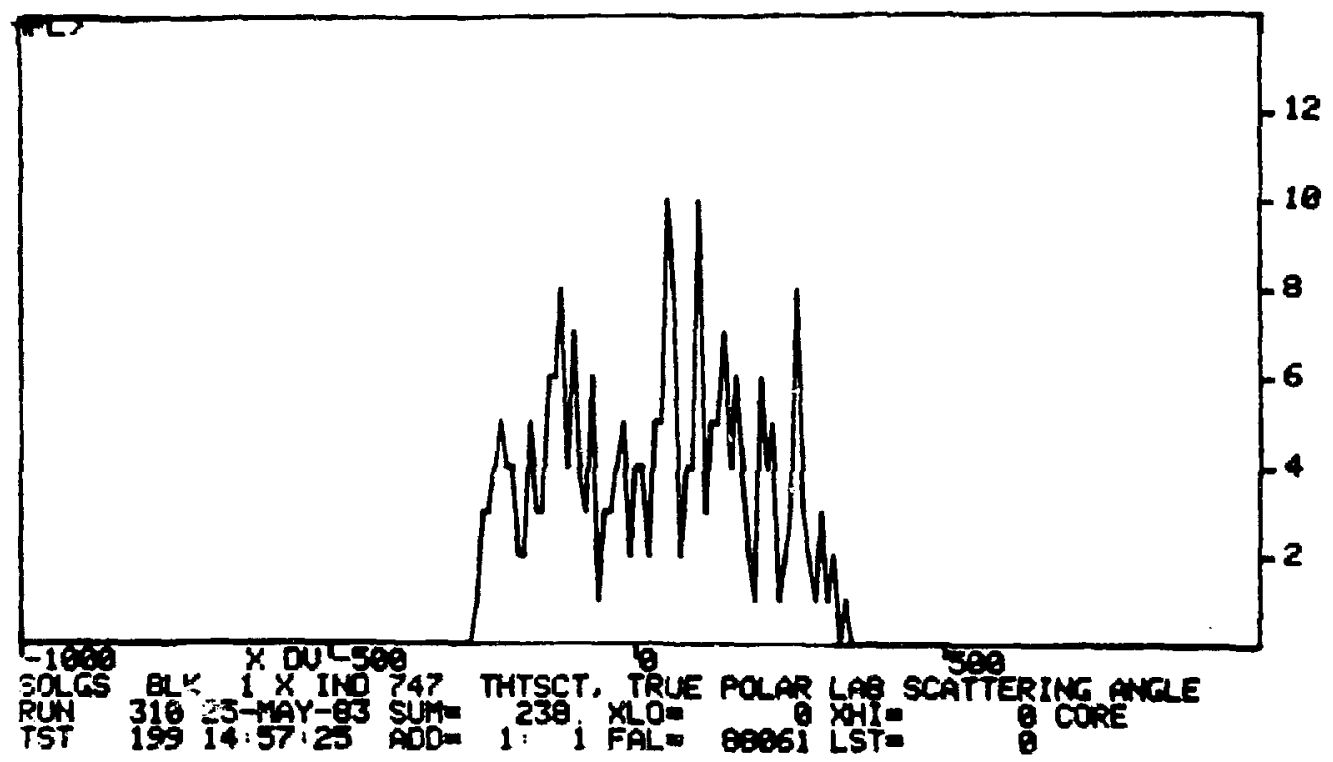

Figure 4.1.b. Histogram SOLGS showing the uncorrected distribution of $\theta_{\mathrm{Lab}}\left(10^{\circ}\right)$ of particles passing good event, ground state gate, and solid angle requirements. Zero in this plot is equivalent to the nominal 10 degree setting of the spectrometer. 


\subsubsection{Energy resolution}

The resolution of a spectrometer system is determined by three factors: 1) the optical properties of the magnet system; 2) the spatial resolution of position measuring detectors; and 3 ) energy loss uncertainties in the passage of the beam through matter. The first contribution can be designed to any order of precision, but often this is not the case--usually second and higher order imperfections are known to exist in the design. These optical effects are correctable in software analysis--but of ten the design TRANSPORT coefficients do not match the measured ones, in which case the spectrometer is said to be "not understood" and the resolution suffers. In the second factor, the finite spatial resolving capability of drift chambers places a limit on achievable energy resolution. Thirdly, the beam and scattered particles are confined to a vacuum system in the spectrometers, so only vacuum windows, scintillators, drift chambers, the target, and air near the target contribute to energy loss uncertainty.

In figure 4.1.1.a, the measured resolution for $x$-position is shown for DC2. This corresponds to uncertainties $\Delta x_{0}=\Delta x=2.1 \mathrm{~mm}$ fwhm $(0.9 \mathrm{~mm} \mathrm{rms})$, and $\theta_{0}=6 \mathrm{mr}$, for the $15.4 \mathrm{~cm}$ chamber separation. The expected, or design resolution is calculated to first order using these parameters together with the Sp2 TRANSPORT coefficients (appendix 3.3.4.a) $\mathrm{R}_{11}=1.06, \mathrm{R}_{12}=0.02$, and $R 16=2.66$, in 


$$
\Delta \delta^{2}=\left(\frac{1}{R_{16}^{2}}\right)\left(R_{11}^{2}\left(\Delta x_{0}\right)^{2}+R_{12}^{2}\left(\Delta \theta_{0}\right)^{2}+(\Delta x)^{2}\right) \cdot \quad \text { eq. 4.1.1.a }
$$

This gives $\Delta \delta=0.067 \%$, or $\Delta E=.39 \mathrm{MeV}$ for kaons at $700 \mathrm{MeV}$. The inclusion of second order terms $(\Delta \delta=.044 \%)$, and the effects of multiple scattering distributions in scintillators(1 MeV, or 0.14\%), drift chambers, etc., added in quadrature, increases this estimate to $1.15 \mathrm{MeV}$. These TRANSPORT coefficients were generated using measured magnet field maps and the assumption that the fringe field extends one-half the magnet aperture radius beyond the magnet. Important optical quantities were therefore included by estimate, not detailed measurement. Achieving this expected resolution was a goal sought through tuning the magnet elements. This estimated optical description resembles reality enough to permit using it as a starting point for an iterative optimization technique known as " $\delta$-fitting".

There were two approaches used in the optical optimization and description of the specirometers: 1) the "LAMPF way", relying on the $\delta$-fitting process, and; 2) the "BNL way", using a matrix inversion of TRANSPORT coefficients generated from detailed magnet maps, to give $\delta$. 
In optimizing the polynomial coefficient fit of $\delta$, the main assumption is an elastic scattering condition, so that $\delta_{\mathrm{Sp} 1}=\delta_{\mathrm{Sp} 2}$. Given $\delta_{\mathrm{Sp} 1}$, and rays written for $\delta_{\mathrm{Sp} 2}=0, \pm 1 \%, \pm 2 \%$, a fit describing $\delta_{\mathrm{Sp} 2}$ is generated. Then a new $\delta_{\mathrm{Sp} 1}$ is defined by $\delta_{\mathrm{Sp} 1}=\delta_{\mathrm{Sp} 2}$, and more rays are written for $\mathrm{Sp} 2$. The process is repeated to convergence. Although it is reasonable to expect success with this technique, there were two problems in the present experiment. First, it is questionable if beginning the process with $7 \mathrm{MeV}$ resolution, the elastic scattering assumption is valid. And second, $\delta$-coefficients for pion elastic scattering at $P_{\pi}=1054$ $\mathrm{MeV} / \mathrm{C}$ descibing $\mathrm{Sp} 1$, and $\delta$-coeŕficients for kaon elastic scattering at $P_{K}=716 \mathrm{MeV} / c$ describing $\mathrm{Sp} 2$, were combined to calculate $\delta$ 's for $\left(\pi^{+}, \mathrm{K}^{+}\right)$events. The large momentum mismatch in this case could have contributed resolution uncertainty through constant terms in the polynomial expressions.

The best energy resolution obtained for any type of scattering was $2.2 \mathrm{MeV}$. This was for pion elastic scattering at $\theta_{\text {Lab }}=10^{\circ}$ with $P_{\pi}=716 \mathrm{MeV} / \mathrm{C}$ on the ${ }^{12} \mathrm{C}$ component of a "finger-shaped" scintillator with dimensions $3 \mathrm{~mm} \times 19 \mathrm{~mm} \times 9 \mathrm{~mm}$ ( $x$ by $y$ by $z$ ). This situation greatly restricted the effective beam phase space, giving a "point source" for scattering. However, for $\left(\pi^{+}, \mathrm{K}^{+}\right)$, count-rate requirements dictated that the full phase-space be utilized with a target encompassing the entire beam. Only an energy resolution limit of greater than $\sim 11 \mathrm{MeV}$ for $\left(\pi^{+}, \mathrm{K}^{+}\right)$using $\delta$-coefficients 
produced with the $\delta$-fitting technique can be stated, since no peaks were seen. Therefore, the $\delta$-fitting technique met with failure. The reason for this was not understood, however, it was suspected that a mismatch of constant-terms in the $\delta$-expansion for the two spectrometers was the culprit. But $\delta$-coefficients (generated from magnet maps) supplied by the BNL members of the collaboration, which had been successfully used in in previous $\left(\mathrm{K}^{-}, \pi^{-}\right)$experiments, yielded the spectra shown in figure 4.1.a, with energy resolution of $-3.2 \mathrm{MeV}$ fwhm. not the resolution design goal, but sufficient for an observation of states in ${ }^{12} \mathrm{C}$, expected to be $-11 \mathrm{MeV}$ apart.

\subsection{Error analysis}

Before differential cross sections can be calculated, experimental errors must be identified and assigned a numerical factor, as they will enter as overall cross section corrections. The following systematic uncertainties exist, and also contribute mulplicative corrections to the cross sections (calculated in section 4.3): 1) drift chamber efficiency ( $\pm 10 \%) ; 2) \mathrm{e}^{+}$and $\mu^{+}$ contamination of the $\pi^{+}$beam $( \pm 17 \%) 3$ ) subtraction of estimated background under the peaks $( \pm 10 \%)$; and 4$)$ uncertainty in kaon decay-length corrections $( \pm 10 \%)$. 
A drift chamber does not always register a hit when it should--it is not $100 \%$ efficient as a detector. Using drift chamber pairs, the efficiency is calculable, since when a hit exists in one chamber of she pair, it should exist in the other; absence of the expected hit is attributed to chamber inefficiency. There are two classes of contributions to chamber inefficiency. The largest is a "geometric" inefficiency, present in the Sp2 front chambers DC5 and DC7. These chambers intercepted some portion of the unscattered incident pion beam; DC5 more than DC7. Therefore, an unscattered particle passing through DC5 might possibly miss DC7 altogether, but scatter on the Sp2 vacuum pipe, loosing sufficient energy to have proper kaon TOF. This condition would appear as an inefficiency in DC7. Moreover, one would expect this geometrical effect to vary with the angle setting of Sp2. The second class of inefficiency is a true inefficiency; it arises from the physics of the chamber, but is also calculable. Finally, note that the percent of inefficiency in the Sp1 chambers (DC1, DC2, DC3) was irrelevant, since the analysis required a beam trigger to have one hit in each of these three chambers, so by definition, the class of beam events for which the chambers were $100 \%$ efficient was selected.

The chamber efficiency calculation for DC5 and DC7 is now shown for $\theta_{\text {Lab }}=5.6^{\circ}$. In this example, the notation $(D C 5, D C 7)$ is used to represent the percentage of the total hits for the specified case of number of hits in DC5 and DC7; that is, $(0,2)=0.2$ means $0.2 \%$ of 
the total hits had zero hits in DCS and two hits in DC7. The zero hit efficiency is defined as the zero hit efficiency in DC5 times the zero hit efficiency in DC7. This joint zero hit efficiency definition is intended to remove geometrical inefficiency effents. Using the notation above, this is written

$$
\mathrm{DCS}_{z}=\frac{(0,1)+(0,2)}{(1,1)+(1,2)+(2,1)+(2,2)} . \quad \text { eq. } 4.2 . \mathrm{a}
$$

Similarly,

$$
\mathrm{DC7}_{z}=\frac{(1,0)+(2,0)}{(1,1)+(1,2)+(2,1)+(2,2)} . \quad \text { eq. } 4 \cdot 2 \cdot \mathrm{b}
$$

Then the joint zero hit probability is

$$
(0,0)=\left(D 5_{z}\right) \cdot\left(D C 7_{z}\right) \cdot \quad \text { eq. } 4 \cdot 2 \cdot c
$$

In table 4.2.a, the results of applying eqs. 4.2.a, .b, and .c to data at all spectrometer angles are shown. Note that for a given angle, the measured percentages do not add to $100 \%$. This is because of small non-zero $(3,1),(2,3)$, etc. contributions, not considered here.

Using data in table 4.2.a, drift chamber efficiencies can be calculated; once certain hit conditions are defined as usable or unusable. Defined as unusable are $(0,0),(2,0),(0,2)$, and $(2,2)$. 
Hit condition $\theta_{l}=5.6^{\circ}(\%) \quad \theta_{l}=10.3^{\circ}(\%) \quad \theta_{l}=15.2^{\circ}(\%)$

$\begin{array}{lrrr}(0,0) \text { meas. } & 2.9 & 14.1 & 30.7 \\ (0,0) \text { calc. } & 0.8 & 2.3 & 3.8 \\ (0,1) & 9.2 & 5.6 & 4.6 \\ (0,2) & 0.2 & 0.3 & 0.2 \\ (1,0) & 6.1 & 18.8 & 22.0 \\ (1,1) & 75.2 & 52.9 & 37.0 \\ (1,2) & 2.0 & 2.1 & 0.8 \\ (2,0) & 0.3 & 1.5 & 1.6 \\ (2,1) & 3.3 & 3.7 & 2.8 \\ (2,2) & 0.7 & 0.9 & 0.3\end{array}$

Table 4.2.a. Drift chamber (DC5 and DC7) hits satisfying specific hit condition as a percent of total hits, where a good hit is defined as (exactly one hit in Sp1 chamivers)AND(good TOF in Sp2).

Usable hits are then $(0,1),(1,0),(1,1),(1,2)$, and $(2,1)$, since all that is needed is one hit in the front of $\mathrm{Sp} 2$ for track reconstruction. Then the drift chamber efficiency at the front of $\mathrm{Sp} 2$ is

$$
E=\frac{\text { usable }}{\text { usable }+ \text { unusable }} . \quad \text { eq. } 4.2 . c
$$

The results of this calculation for the three spectrometer settings are $\mathrm{E}_{\mathrm{f}}\left(5.6^{\circ}\right)=98.0 \%, \mathrm{E}_{\mathrm{f}}\left(10.3^{\circ}\right)=94.3 \%$, and $\mathrm{E}_{\mathrm{f}}\left(15.2^{\circ}\right)=91.9 \%$. The 
therefore persists, probably because of geometrical effects present in cases not calculated here -- but this problem should be unraveled in the future with data taken specifically for that purpose.

The efficiency in the Sp2 rear wire chambers PC3 and PC4 is defined in the same way as for the front drift chambers, except that only the condition $(1,1)$ is defined as usable, since this information must be used to project backwards through $\mathrm{Sp} 2$ in calculating $\theta_{\mathrm{Sp} 2 \text {, front }}$. The resultinf Sp2 rear efficiencies are $E_{r}\left(5.6^{\circ}\right)=91 \%, E_{r}\left(10.3^{\circ}\right)=85 \%$, and $E_{r}\left(15.2^{\circ}\right)=82 \%$.

Combining these efficiency results yields an overall chamber efficiency for $S p 2$ given by $E_{T}=E_{f} \cdot E_{r}$. The results are $\mathrm{E}_{\mathrm{T}}\left(5.6^{\circ}\right)=89 \%, \mathrm{E}_{\mathrm{T}}\left(10.3^{\circ}\right)=80 \%$, and $\mathrm{E}_{\mathrm{T}}\left(15.2^{\circ}\right)=75 \%$. It is these percentages that enter into the cross section as corrections. Since there is roughly a $\pm 10 \%$ variation in the efficiencies, that is the uncertainty assigned to their determination.

A large correction to the cross section and also the greatest uncertainty, is attributed to an $e^{+}$contamination of the incident $\pi^{+}$ beam. This was discovered during data analysis, after the completion of the experiment. The contamination was an inherent quality of the beam, and therefore could not have been eliminated, but could have been measured more precisely if it had been discovered during the experiment. So the analysis here is unfortunately crude. 
For a given momentum, $\pi^{+}, \mu^{+}$, and $\mathrm{e}^{+}$hava differing TOF through the system. At the incident beam momentum of $1054 \mathrm{MeV} / \mathrm{c}$, these three particle species all have $\beta>.99$, so the TOF difference is not measurable. But at the lower $P_{\text {beam }}=716 \mathrm{MeV} / \mathrm{c}, \beta_{\pi}=.982$, while $\beta_{e}=.999$, so that over the $12.59 \mathrm{~m}$ flight path from $\mathrm{S} 1$ to $\mathrm{s} 3$, one expects a TOF difference of $.79 \mathrm{~ns}$ between $\mathrm{e}^{+}$and $\pi^{+}$. With a TOF resolution of about $1 \mathrm{ns,}$, the TOF spectrum would not show two distinct peaks, but rather would display a skewing to the fast TOF side associated with the $e^{+}$component of the $\pi^{+}$beam. The $\mu^{+}$ component would not be observable with this technique, but it is shown later that there is good reason to believe the $\mu^{+}$ contamination was small.

Since the presumed $e^{+}$and $\pi^{+}$peak positions are nearly the same at $P_{\text {beam }}=1054 \mathrm{MeV} / \mathrm{c}$, the TOF spectrum at this momentum was used to establish a reference peak shape by fitting to a Gaussian distribution.

Using

$$
G(x)=A \cdot \exp \left\{-\left(\frac{x-\mu}{f}\right)^{2}\right\},
$$

the fit shown in figure 4.2.a was obtained with $\mu=2.07$ ns and $f=(.87 n s)(.603)=.53$ ns. The nominal software conversion was 2 units/1 ps, but by looking at the TOF peak shift as a function of momentum, this conversion was found to be 2.38 units/1 ps, because 
$P=1053.6 \mathrm{MeV} / \mathrm{C}$

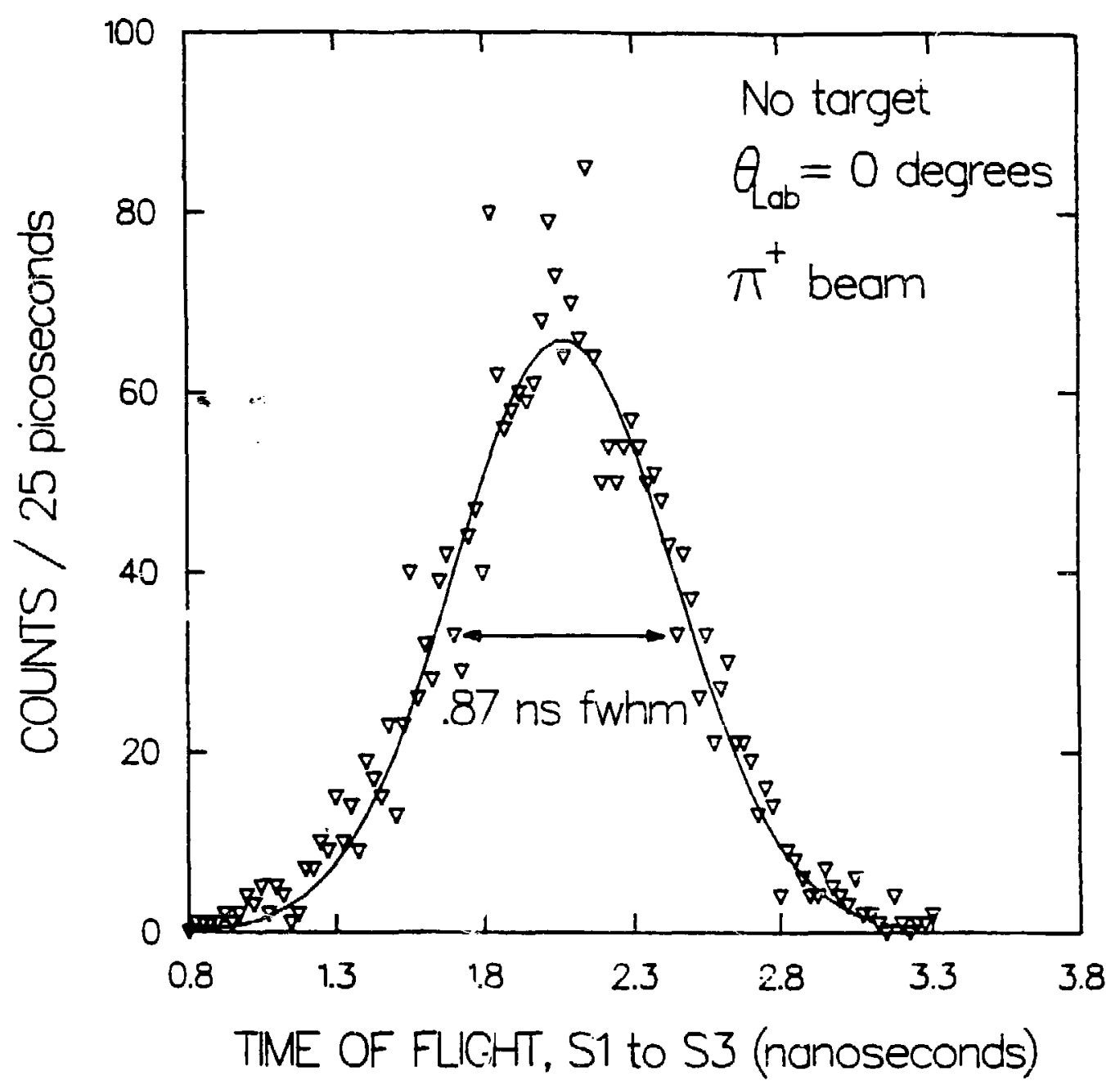

Figure 4.2.a. Time of flight distribution from $S 1$ to $S 3$, no target, $\pi$-beam. This peak vas used to establish a standard line-shape for use in the beam contamination calculation. 
of TDC calibration. Using the corrected relative positions for $\mathrm{e}^{+}$ and $\pi^{+}$at $P_{\text {beam }}=716 \mathrm{MeV} / \mathrm{c}$, and the reference Gaussian line shape, the fit in figure 4.2.b was obtained. The amplitudes are $A_{e}=42$ and $A_{\pi}=110$, so that the percent of $e^{+}$contamination in the beam was 28\%. But this was the contamination at $P_{\text {beam }}=716 \mathrm{MeV} / \mathrm{c}$, not $P_{\text {beam }}=1054 \mathrm{MeV} / \mathrm{c}$, the actual beam momentum. Since this type of TOF measurement cannot distinguish between $\mathrm{e}^{+}$and $\pi^{+}$at $1054 \mathrm{MeV} / \mathrm{C}$, the only resort is to state that: the percent contamination is known to fall with rising momentum [Am 82], there "qre the contamination is estimated to be $14 \pm 14 \%$, taking the mid-point in an extrapolation to zero.

The estimate of $\mu^{+}$contamination is essentially a back-of-the-envelope calculation. Over the approximately $17 \mathrm{~m}$ flight path froin the production target to the scattering target, only about $25 x$ of the pions decay to muons. Since the acceptance solid angle of $\mathrm{Sp} 1$ is less than $20 \mathrm{msr}$, a very liberal estimate of $\mu^{+}$ contamination assigns a $6 \%$ level. Together with $\mathrm{e}^{+}$contamination, this brings the total beam impurity to $17 \pm 17 \%$.

The background was determined subjectively by extrapolating a straight line from the unphysical region of $E_{e x} \leq-2 \mathrm{MeV}$ to the continuum region. No physics is contained in the estimate. In the $\theta_{L}=10.3^{\circ}$ spectrum, the background estimate is about 10 events $/ 1$ $\mathrm{MeV}$ bin. The uncertainty assigned to this is $\pm 20 \%$, equivalent to \pm 2 events. 


\section{POSIRON CONTAMINATION IN $\pi^{+}$BEAM}

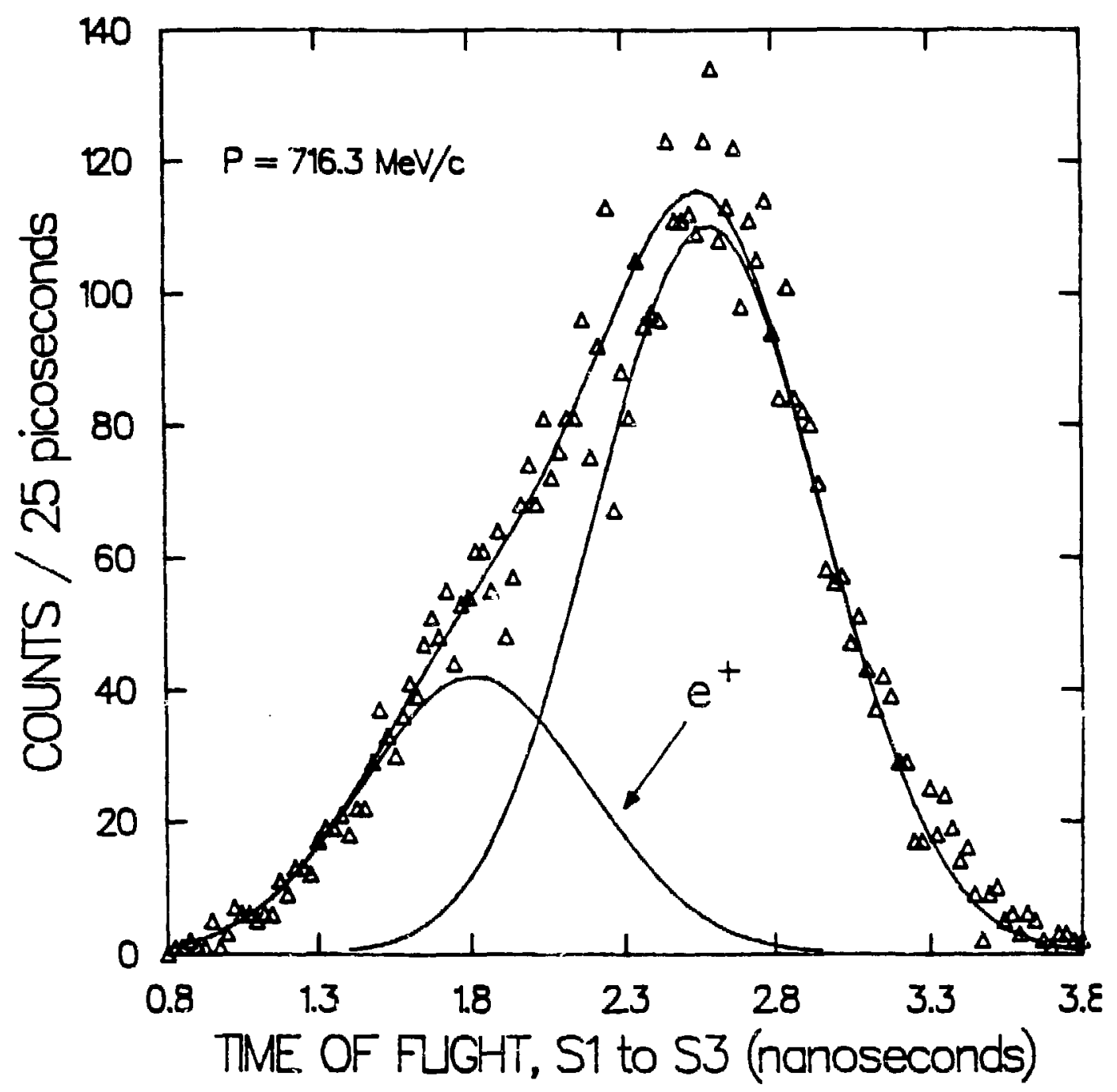

Figure 4.2.0. Fit to TOF distribution at $716 \mathrm{MeV} / \mathrm{C}$ using the line-shape shown in figure 4.2.a. The $e^{+}$contamination of the $\pi^{+}$ beam is revealed as a shoulder on the fast TOF side of the c'rrve. 
The largest correction to the cross section arises from the in-fiight decay of kaons in $\mathrm{Sp} 2$ from the scattering target to the $\mathrm{LN}_{2}$ Cerenkov counter. Only about $23.3 \%$ of the kaons survived over the $7.85 \mathrm{~m}$ central path flight length, so that the cross section must be multiplied by 4.29 to correct for decay. There are two classes of error in the determination of the decay-length correction: 1) $\ell=7.85 \mathrm{~m}$ is the central path length, but there are many others; 2) particle identification becomes less reliable if the particle decays near one of the detectors in the rear of the system. The first class of error can be estimated by calculating the maximum first order path difference in Sp2:

$$
\Delta l=R_{51} x_{0}+R_{52} e_{0}+R_{56} \delta, \quad \text { eq. 4.2.e }
$$

using TRANSPORT coefficients from appendix 3.3.4.a. This gives $\Delta l= \pm .12 \mathrm{~m}$, or $\pm 2 \%$ error in the decay-length correction of 4.3 . The second class of error arises from particle misidentification: consider a kaon that decays within the Cerenkov counter volume. It is not clear how far the $K$-decay products must travel in the $\mathrm{LN}_{2}$ volume to generate a signal. The demarcation of the Sp2 end-point is therefore ambiguous with respect to what value is used to calculate the decay-length. Assuming a liberal value of $\pm 50 \mathrm{~cm}$ in the knowledge of the dezay-length of the kaon contributes $\pm \%$ error in decay correction, so that overall, a $\pm 10 \%$ error is assigned. In 
future experiments, this problem could be studied in greater detail by examining the measured decay rate with no target at $\theta_{L}=0^{\circ}$, as a function of kaon momentum.

In summary, the various data corrections and errors are: 1) drift chamber efficiency $( \pm 10 \%) ; 2) \mathrm{e}^{+}$and $\mu^{+}$contamination of the $\mathbf{n}^{+}$beam $(17 \pm 17 \%)$; 3$)$ subtraction of the estimated background under the peaks ( $\pm 20 \%) ;$ and 4 ) uncertainty in decay-length corrections ( $\pm 10 \%)$. 


\subsection{Differential cross sections}

The scattering probability can be expressed as,

$$
\frac{d I_{s}}{d \Omega}=F N \frac{d \sigma}{d \ell},
$$

eq. 4.3.a

where $I_{S}$ is the number of scattered particles, de is the solid angle subtended by the detector, $F$ is the incident flux, $N$ is the number of scattering centers in the target per unit volume, and $d \sigma$ is a constant of proportionality called the differential scattering cross section, having units of area. Practical expressions for $\mathbf{F}$ and $\mathbf{N}$ are, $F=I_{0} / a$ where $I_{0}$ is the number of incident particles and $a$ is the beam area, and $N=a\left(N_{0} p / A\right) d$ where $N_{0}$ is Avogadro's number, $p$ is the target density, $A$ is the target atomic weight, and $d$ is the target thickness. By suitable arrangement of these quantities, the usual expression for the scattering cross section is written

$$
\frac{d \sigma}{d \Omega}=\frac{1}{d \Omega}\left(\frac{I_{s}}{I_{0}}\right)\left(\frac{A}{N_{0} \rho d}\right) \cdot \quad \text { eq. } 4 \cdot 3 \cdot b
$$

For this particular experiment, several cross section correction factors have been discussed which must be incorporated into eq. 4.3.b. They are corrections for: 1) kaon decays in $\mathrm{Sp} 2$ (D); 2) total chamber efficiency $\left(E_{\mathrm{T}}\right)$; and 3$) \mathrm{e}^{+}$and $\mu^{+}$contamination of the $\pi^{+}$beam (C). Including these factors, eq. 4.3.b becomes 


$$
\frac{d \sigma}{d Q}=\frac{1}{d Q}\left(\frac{I_{S}}{I_{0}}\right)\left(\frac{A}{N_{0} \rho d}\right)\left(\frac{D}{E_{T} C}\right) \cdot \quad \text { eq. } 4.3 . c
$$

Only $I_{S}, I_{0}$, and $E_{T}$ varied with angle, and will be presented shortly in tabular form. The "constant" factors have been previously discussed, but are restated here. The solid angle, dg, was defined by a software cut (which good events were required to pass) at the front of Sp2 with $\theta=-27$ to $+27 \mathrm{mr}$ and $\phi=-80$ to +80 $\mathrm{mr}$ for totals of $\Delta \theta=54 \mathrm{mr}$ and $\Delta \phi=160 \mathrm{mr}$. This gives $\mathrm{d} \Omega=(\Delta \theta)(\Delta \phi)=8.64 \times 10^{3} \mathrm{mr}^{2}=8.64 \times 10^{-3} \mathrm{str}$. As discuse ed in section 3.6, (A/Nopd) $=8.39 \times 10^{-24} \mathrm{~cm}^{2}$, with $p$ for the carbon component of the scintillator target. To account for kaons missing from the good event spectrum because of decay over the $7.85 \mathrm{~m}$ flight path in Sp2, $D=4.29$. Finally, the percent of actual $\pi^{+}$particles in the incident beam $I_{0}$ is included in the factor $C=.83$, arising from a subiraction of the deduced $17 \% \mathrm{e}^{+}$and $\mu^{+}$beam contamination. The scatiering differential cross sections are presented in table 4.3.a, along with the various angle-dependent values.

Figure 4.3.a shows the differential cross sections for the ground state and excited state multiplets, along with a theoretical cross section curve discussed in the following chapter. 
$\theta(\mathrm{deg}) \quad E_{e x}(\mathrm{MeV}) \quad \mathrm{d} \sigma / \mathrm{d} \Omega(\mu \mathrm{b} / \mathrm{str}) \quad I_{s} \quad I_{0} \quad E_{T}$

\begin{tabular}{rrrrrrr}
\hline 5.6 & 0 & 13.12 & 55 & $2.36 \times 10^{10}$ & .89 \\
& 11 & 16.46 & 69 & & & \\
10.3 & 0 & 9.07 & 156 & $1.08 \times 10^{11}$ & .80 \\
& 11 & 9.24 & 159 & & & \\
15.2 & 0 & 5.26 & 14 & $1.78 \times 10^{10}$ & .75 \\
& 11 & 4.51 & 12 & & &
\end{tabular}

Table 4.3.a. Differential scattering cross sections and angle-dependent values for variables in eq. 4.3.c. 


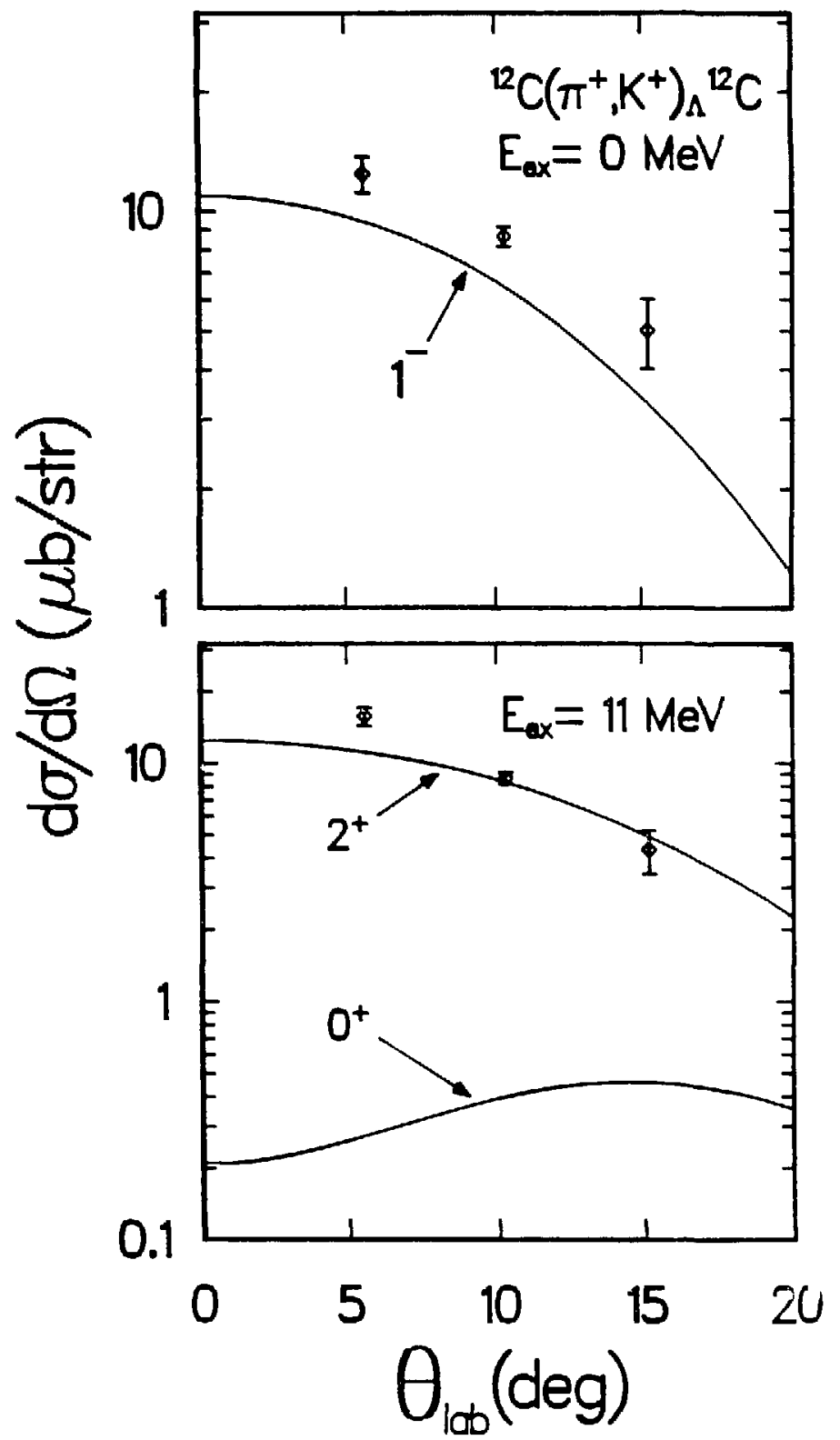

Figure 4.3.a. Differential cross sections for the multiplet peaks near $0 \mathrm{MeV}$ and $11 \mathrm{MeV}$ in $\mathrm{E}_{\mathrm{ex}}$. 
CHAPTER FIVE

5. Theoretical calculation of the cross sections

The theoretical calculation of the ${ }^{12} \mathrm{C}\left(\pi^{+}, \mathrm{K}^{+}\right){ }_{\Lambda}{ }^{12} \mathrm{C}$ differential cross sections follows the spirit of the $\left(\mathrm{K}^{-}, \pi^{-}\right)$p-shell calculations performed by Auerbach et al [Au 83]. First, expected shtil model couplings were identified in a simple $\wedge$-particle, n-hole picture. Then, using a DWBA program CHUCK [Ku 78], cross sections were calculated for all the specified particle-hole configurations. The potentials used in this calculation came from fitting elastic $\pi^{+}$ and $\mathrm{K}^{+}$scattering. A major correction to this calculation arose from averaging over the Fermi-momentum distribution of the struck neutron, an important consideration in the sharply peaked $\left(\pi^{+}, K^{+}\right)$ reaction.

5.1 Shell model configurations

The ${ }^{12} \mathrm{C}\left(\mathrm{\pi}^{+}, \mathrm{K}^{+}\right) \Lambda^{12} \mathrm{C}$ reaction proceeds by converting an $\mathrm{s}-$ or p-shell neutron to a $\Lambda$ which is free to occupy any s-, p-, or s-d-shell state, since it is not excluded from any state by the Pauli principle. Several of the possible neutron-hole, $\wedge$-particle couplings may be eliminated from the outset. These are states 
coupling to unnatural parity, in all cases calculated to have little strength; spin-flip is required, and the states are suppressed.

Energetically, the ground state configuration is $\left(\Lambda^{s_{1} / 2} \times{ }_{n} \mathrm{p}^{-1} 3 / 2,1 / 2\right)$. This coupling gives rise to two $\mathrm{J}^{\pi}=1^{-}$ states, in $\left({ }_{\Lambda} s_{1 / 2} \times{ }_{n} p^{-1}{ }_{3 / 2}\right)$ and $\left({ }_{\Lambda} s_{1 / 2} \times{ }_{n} p^{-1}{ }_{1 / 2}\right)$, depending on the state of the neutron hole. The excited staie multiplet at $E_{e x}=11$ $\mathrm{MeV}$ results from the configuration $\left({ }_{\Lambda} \mathrm{p}_{3 / 2,1 / 2} \times \mathrm{n}^{\mathrm{p}^{-1}} 3 / 2,1 / 2\right)$ coupling to three $\mathrm{J}^{\pi}=2^{+}$states and two $\mathrm{J}^{\pi}=0^{+}$states.

In the 5.9 degree spectrum it is interesting to note weak evidence for a peak appearing near an excitation of about $18 \mathrm{MeV}$. There are several excitations which might plausibly appear at that energy; the deeply-lying $\left({ }_{\Lambda} s_{1 / 2} \times n^{s^{-1}}{ }_{1 / 2}\right)$ substitutional state is one ard the $\left({ }_{\Lambda} d_{5 / 2,3 / 2} \times n_{n} p^{-1} 3 / 2,1 / 2\right)$ is another. DWBA predicions strongly favor the iatter candidate, but a definite identification awaits further experimental work. Since the quasi-free spectrum in the $\left(\pi^{+}, K^{+}\right)$reaction is broader than the more peaked distribution in the quasi-free region of the $\left(\mathrm{K}^{-}, \pi^{-}\right)$reaction, such continuuin hypernuclear states, if they exist, might be more clearly observed in the $\left(\pi^{+}, K^{+}\right)$reaction [Do 80$]$.

The main goal of this experiment was to show that the $\left(\pi^{+}, \mathrm{K}^{+}\right)$ reaction preferentially excites the higher spin $2^{+}$states in a given shell model configuration, complementing the lower-spin admixture of the $\left(\mathrm{K}^{-}, \pi^{-}\right)$reaction. Such a difference would not be evident in the ground state, but in the excited state multiplet one expects to 
observe differences in the spin states for the two reactions since both $2^{+}$and $0^{+}$are possible. Support for this conjecture must come from theoreticaily predicted cross section strengths and distriblitions, as no experimental measurement of $\mathrm{J}^{\pi}$ was sought via observation of de-excitation $\gamma$-rays.

\subsection{The DWBA}

The distorted-wave-Born-approximation (DWBA) computer program CHUCK [Ku 78] was used to calculate the cross sections, closely following the calculations performed by Auerbach et al [Au 83] for the $\left(\mathrm{K}^{-}, \pi^{-}\right)$reaction on a series of $\mathrm{p}$-shell nuclei. In this approach, potential parameters which fit $\pi^{+}$-elastic scattering were used to describe the incoming channel, while potential parameters fitting $\mathrm{K}^{+}$-elastic scattering were used to describe the outgoing channel. In both cases the elastic data was from the experiment of Marlow et al [Ma 82] [Ma 84], performed at the BNL C2 beam line. Fits to this data are shown in figures 5.2.a and .b. The optical model parameters shown in the figures were the ones used ir: the CHUCK calculation. Input decks for the various calculations employing nuclear structure information as in reference [Au 83] are included in appendix 5.2.a. CHUCK calculates only the form factor $\left|\mathrm{T}^{\mathrm{B}}{ }_{\text {if }}\right|^{\bar{z}}$ in equation 2.1 of reference $[\mathrm{Au} 83$ ], so table 5.2.a gives the results of the CHuCK calculation for $\mathrm{J}^{\pi}=1^{-}, 0^{+}$, and $2^{+}$, after multiplication by the remaining kinematic quantities. These 


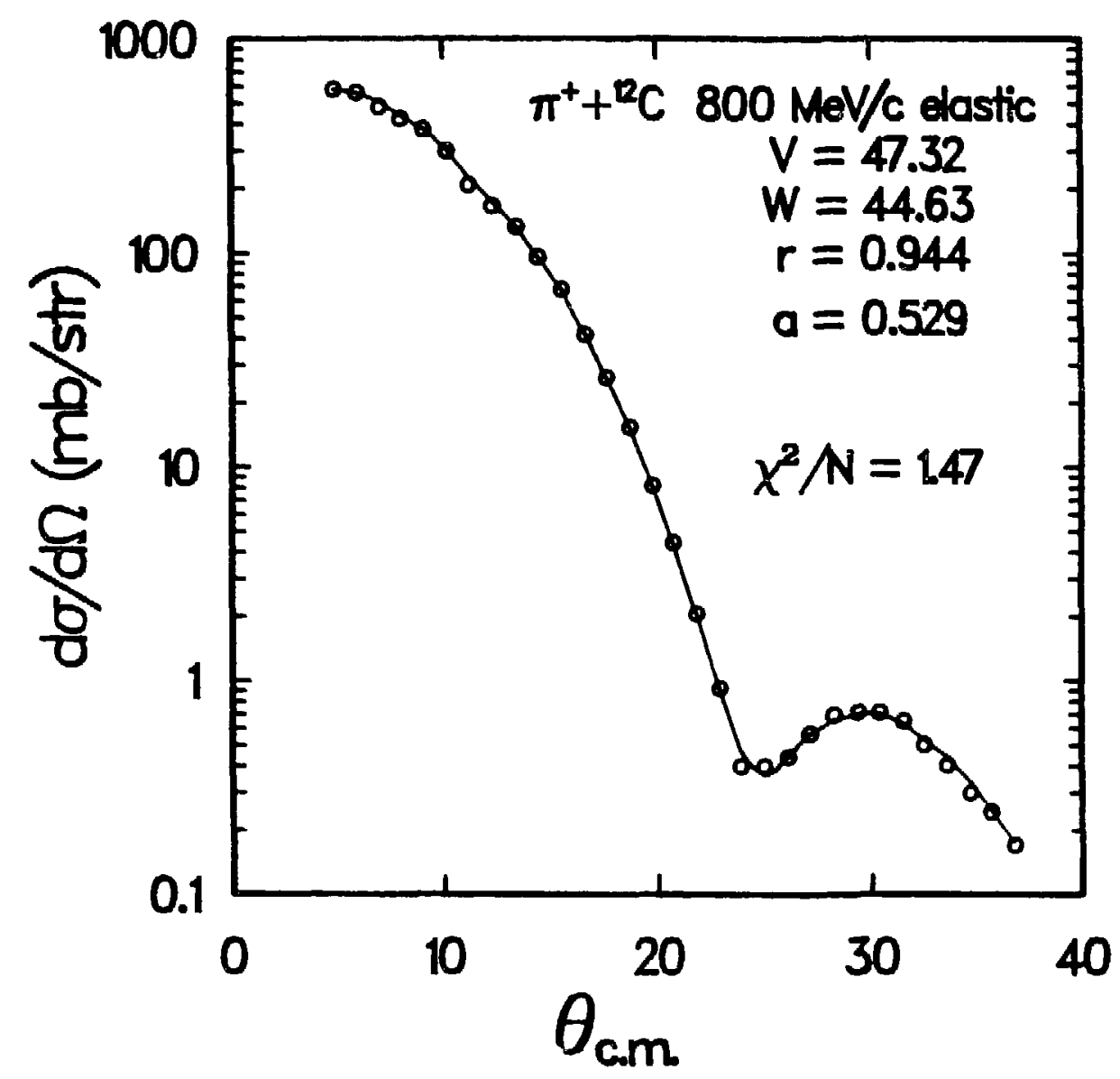

Figure 5.2.a. Optical model fit to the $\pi^{+}$-elastic $800 \mathrm{MeV} / \mathrm{c}$ data of Marlow et al [Ma 84]. 


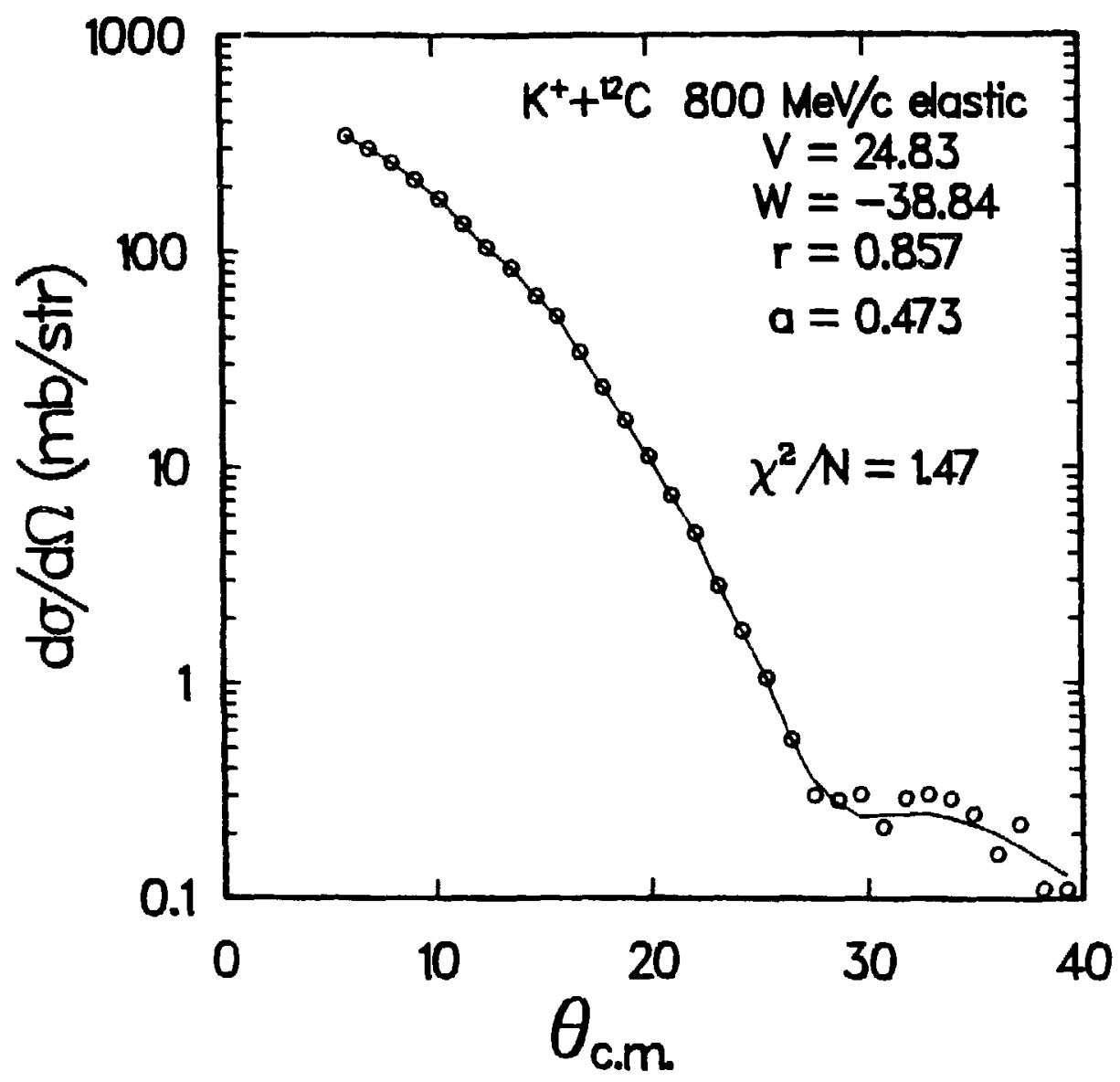

Figure 5.2.b. Optical model fit to the $\mathrm{K}^{+}$-elastic $800 \mathrm{MeV} / \mathrm{c}$ data of Marlow et al [Ma 82]. 
corrections include $J=1.43$ (the Jacobian), $(A /(A-1))^{6}=1.69$, Fermi-factor of .53 (see next section), and spectroscopic factors for the various states: $1^{-}, 0.615 ; 2^{+}, 0.57 ; 0^{+}, 0.64$. Note that the program coherently adds the scattering form factors for each $J^{\pi}$ component in a multiplet before squaring to give do/de. The

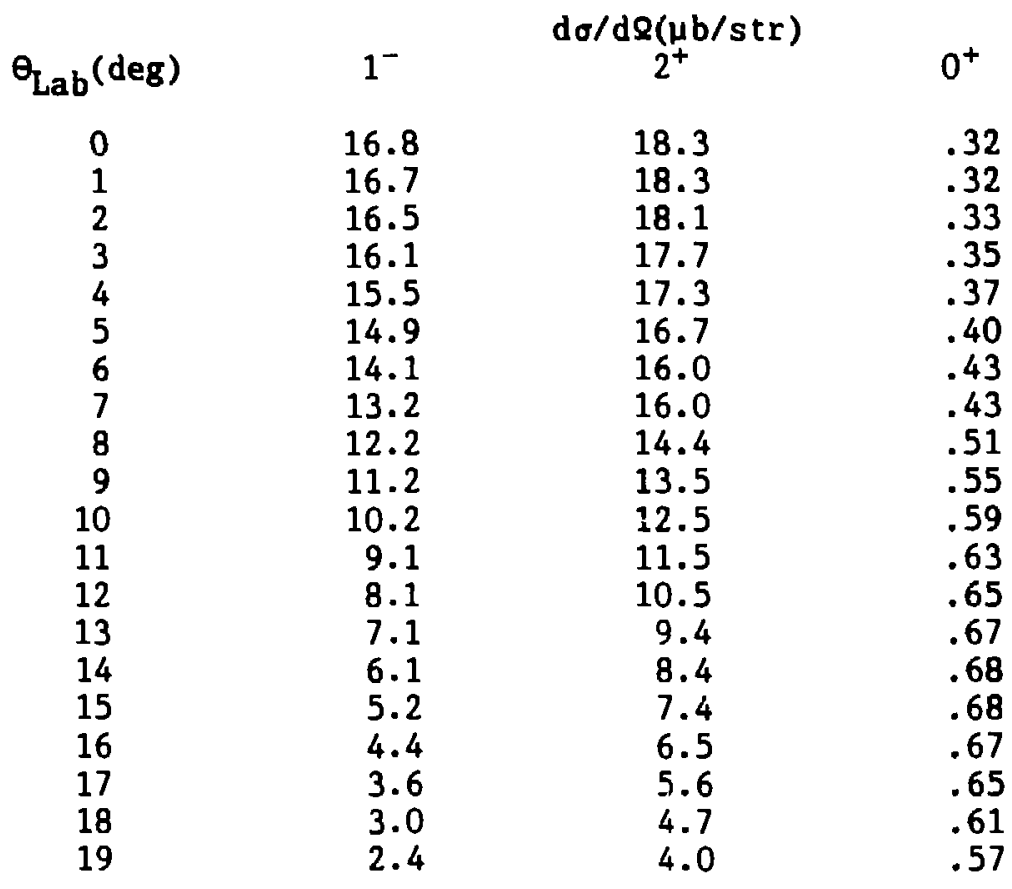

Table 5.2.a. Results of the DWBA calculations after applying corrections described in the text. 
smallness of the magnitude of the $0^{+}$distribution is seen as a confirmation of the higher spin selectivity of the $\left(\pi^{+}, \mathrm{k}^{+}\right)$reaction.

\subsection{Fermi-momentum averaging}

Because the peak in the elementary $\pi^{+}+n \rightarrow \Lambda+k^{+}$cross section as a function of momentum is narrower than the average neutron Fermi-momentum, it is expected that the distribution of the cross section is broader in the nucleus. The CHUCK calculation was performed using the two-body value of $0.95 \mathrm{mb} / \mathrm{str}$ as the forward peak cross section. "Fermi-broadening" was accounted for afterwards by a simple kinematics calculation: for each incident $\pi^{+}$momentum in a range $700-1300 \mathrm{MeV} / \mathrm{c}$, incrementing through the Fermi-momentum distribution, the cross section was computed for that center-of-momentum case anu' stored in a momentum array. The resulting cross section peak strength gives the reduction in $\left(\pi^{+}, \mathrm{K}^{+}\right)$ strength caused by Fermi-momentum broadening. Three momentum distributions were used: 1) uniform, with $K_{f}=220 \mathrm{MeV} / \mathrm{c}$; 2) Woods-Saxon, $p(k)=p_{0}\left(1+\exp \left(k-k_{0}\right) / \Delta k\right)^{-1}$, where $k_{0} \quad 100 \mathrm{MeV} / \mathrm{c}$ and $\Delta k=50 \mathrm{MeV} / \mathrm{c}$; and 3) Gaussian, $\rho(k)=\rho_{0}(\mathrm{~kb})^{2} \exp \left(-\mathrm{k}^{2} \mathrm{~b}^{2}\right)$, with $b=1.64 \mathrm{fm}$. These distributions were normalized so that $2 \int_{0}^{\infty} \mathrm{dkkk}^{2} p(k)=1$. The results of this calculation reduce the elementary peak of $0.95 \mathrm{mb} / \mathrm{str}$ in the three different calculations to: 1) 0.49 ; 2) 0.50 ; and 3$) 0.47 \mathrm{mb} / \mathrm{str}$. Accordingly, the final 
cross section calculations displayed along with the data in figure 4.3.a include the Fermi-averaging factor $.50 / .95=.53$. 
CHAPTER SIX

\section{Conclusion}

There were two goals of the $\left(\pi^{+}, K^{+}\right)$experiment: determine if a hypernuclear signal could actually be seen in the high $\pi^{+}$ background-flux environment, and attempt to verify the expectation of preferential population of higher spin states. The first goal was clearly met with the observation of two strong spectral peaks. And within the framework of a DWBA calculation, the other objective of demonstrating the higher spin selectivity was achieved. Since only ${ }^{12} \mathrm{C}$ was studied, the systematics of high-spin hypernuclear states cannot be discussed, but the reaction was establishad as a promising tocl for studying heavier nuclei in the future.

\subsection{Comparison with $\left(K^{-}, \pi^{-}\right)$reaction results}

Further evidence for higher spin preference of the $\left(\pi^{+}, K^{+}\right)$ reaction can be established by comparison with the $\left(K^{-}, \pi^{-}\right)$results on the same nucleus. Figure 6.1.a shows the measured and calculated cross sections for the ${ }^{12} \mathrm{C}\left(\mathrm{K}^{-}, \pi^{-}\right){ }_{\Lambda}^{12} \mathrm{C}$ reaction [Ch 79]. In both reactions, the $\mathrm{J}^{\pi}=1^{-}$ground state is produced by a $\Delta \mathrm{L}=1$ transition transforming a p-shell neutron into an $s_{1 / 2}$ lambda. But 
in the $\left(\mathrm{K}^{-}, \pi^{-}\right)$reaction, the $E_{e x}=11 \mathrm{MeV}$ peak is belleved to consist of [Ch 79] [Do 79] several unresolved contributions with $\mathrm{J}^{\pi}$ of $0^{+}$and $2^{+}$arising from the $\left({ }_{\Lambda} p_{3 / 2,1 / 2} \times{ }_{n} p_{3 / 2,1 / 2}^{-1}\right)$ coupling. The calculated curves displayed in figure 6.1.a show how the components are believed to separately contribute to the observed total strength. Because the momentum transfer in the $\left(\mathrm{K}^{-}, \pi^{-}\right)$reaction is small, the $\Delta \mathrm{L}=0, \mathrm{~J}^{\pi}=0^{+}$substitutional component dominates at zero degrees. At larger angles, a shoulder develops, corresponding to the $2^{+}$strength, so that the ground state and $11 \mathrm{MeV}$ substitutional state are populated with comparable intensities. The situation in the $\left(\pi^{+}, K^{+}\right)$reaction, where the momentun transfer is higher, is similar to the larger angle $\left(\mathrm{K}^{-}, \pi^{-}\right)$data, in that the 0 $\mathrm{MeV}$ and $11 \mathrm{MeV}$ peaks are of comparable strength (but at all angles). This leads to the conclusion that in the $\left(\pi^{+}, \mathrm{K}^{+}\right)$reaction, the 11 MeV peak is a relatively pure multiplet of same $J^{\pi}$ states, since the ratio of the intensity of the cross section with the ground state is relatively constant, and the ground state is known to be a nearly pure $\mathrm{J}^{\pi}=1^{-}$multiplet. Therefore, the $\mathrm{J}^{\pi}=2^{+}$assignments are made in the $\left(\pi^{+}, \mathrm{K}^{+}\right)$reaction, while $\mathrm{J}^{\pi}=0^{+}, 2^{+}$assignments are made in the $\left(K^{-}, \pi^{-}\right)$reaction, in agreement with the expected higher spin selectivity of the $\left(\pi^{+}, K^{+}\right)$reaction. 


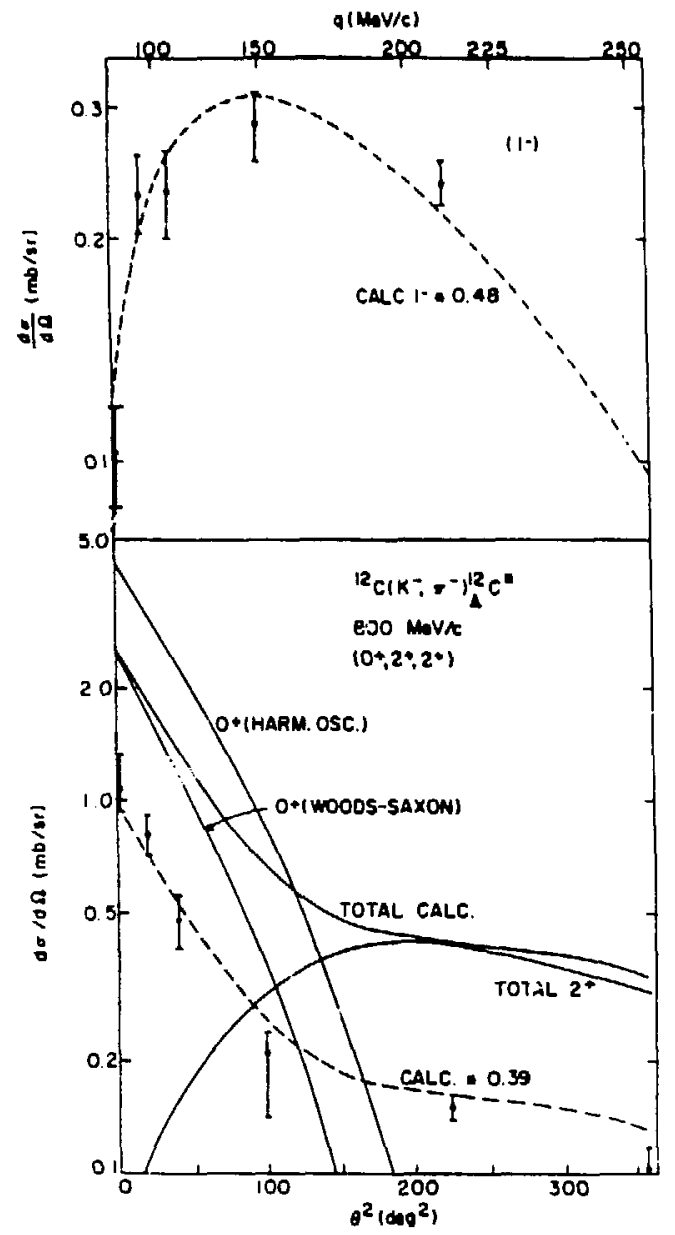

Figure 6.1.a. Angular distributions (lab) calculated for ${ }^{12} \mathrm{C}\left(\mathrm{K}^{-}, \pi^{-}\right){ }_{\Lambda}^{12} \mathrm{C}$ at $800 \mathrm{MeV} / \mathrm{C}$ compared to the data [Ch 79]. 


\subsection{Future prospects}

The next step in using the $\left(\pi^{+}, \mathrm{K}^{+}\right)$reaction should be a systematic study of heavier nuclei, beyond the p-shell. These hypernuclei have not been studied because their ground states, in particular, are usually non-substitutional states of larger $\mathrm{J}$, and therefore not well matched to the momentum transfer characteristic of the $\left(K^{-}, \pi^{-}\right)$reaction. In ${ }^{28} \mathrm{Si}$, for example, the ground state is a $J^{\pi}=2^{+}$state in the $\left.\left(\Lambda_{1}^{s_{1} / 2} \times n_{5 / 2}\right)^{-1}\right)$ configuration. Also in that nucleus, a possible substitutional state formed in the $\left(\Lambda^{d_{5 / 2}} \times n^{d_{5 / 2}^{-1}}\right)$ configuration with $J^{\pi}=4^{+}$might be observable -approaching the category of a truly high spin state.

Figure 6.2.a shows a remarkable feature of the total accumulated hypernuclear data -- $\Lambda$ binding energies have not been reliably measured for $A>15$ (the $A=40$ measurement is a limit, stated for A approximately 40). Clearly, with the $\left(\pi^{+}, \mathrm{K}^{+}\right)$reaction, otherwise inaccessible binding energy measurements are possible, leading to a systematic study of hypernuclear properties beyond the p-shell, and possibly new physics.

A natural extension of the present work would be the study of $A\left(\pi^{+}, K^{+}\right) \Lambda^{*}$ reactions, where $\Lambda^{*}(1520)$ is the obvious first choice because of a narrow $(15.5 \pm 1.5 \mathrm{MeV})$ decay-width. One can envision a research program analogous to the present-day $\Delta$-nucleus work. It is possible that the decay-width is significantly suppressed in the nuclear environment -- as is the case in $\Sigma$-hypernuclei -- further 


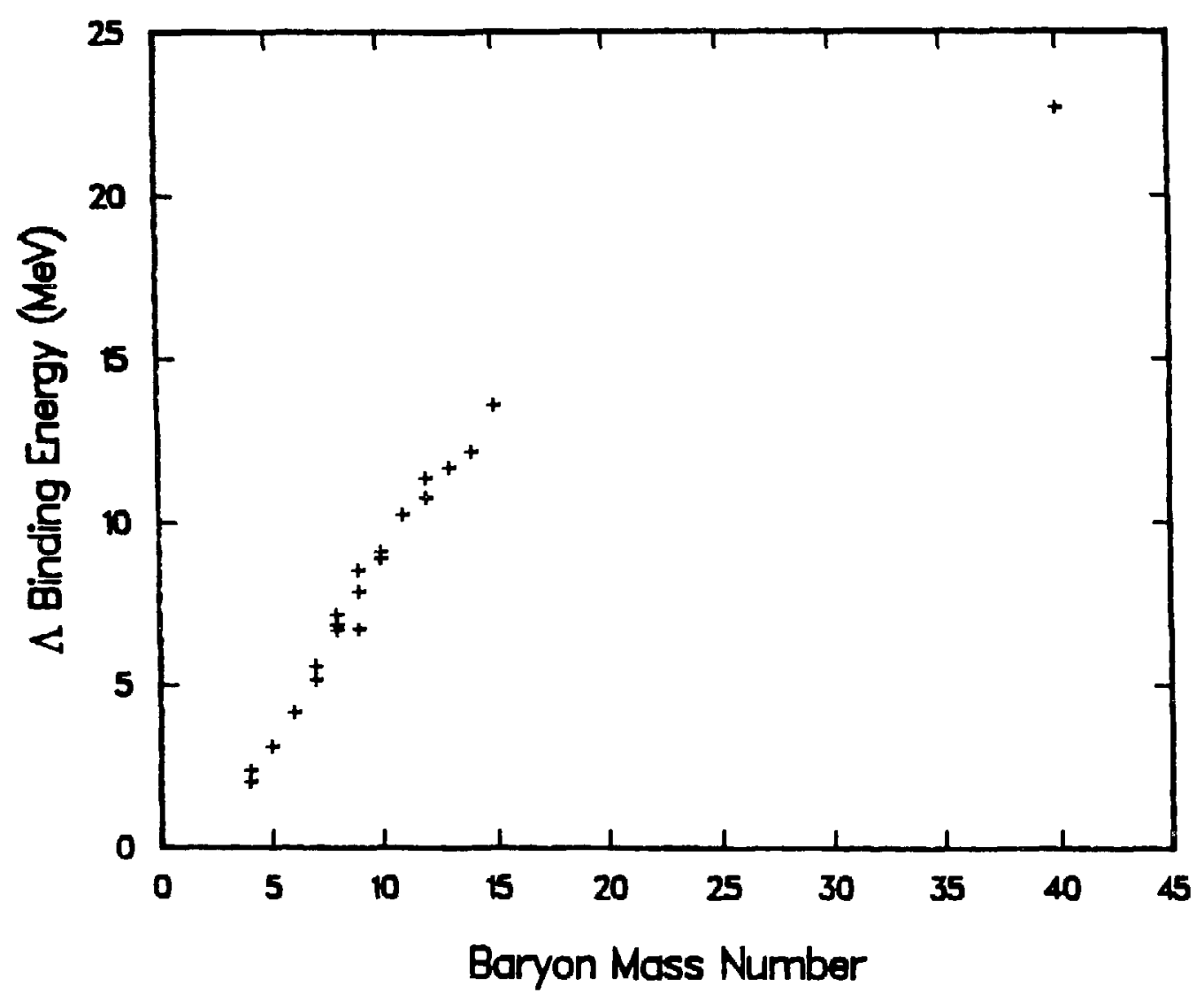

Figure 6.2.a. $\Lambda$ binding energies as a function of Baryon mass number of the nucleus [Pn 72]. 
improving the experimental situation. This kind of experiment will not be possible until the establishment of a meson beam line at higher momenta than presently available -- in the $2 \mathrm{GeV} / \mathrm{c}$ range. Also, improved background rejection techniques and better spectrometer resolution will be necessary.

\subsection{Summary}

In conclusion, the viable production of hypernuclei is achievable with the $\left(\pi^{+}, K^{+}\right)$reaction. The observed cross sections are significantly lower than for the $\left(\mathrm{K}^{-}, \pi^{-}\right)$reaction, but this is compensated by the larger available beam flux. A comparison of the observed cross sections with theoretical predictions is satisfactory. Calculations indicate that the larger momentum transfer of the $\left(\pi^{+}, \mathrm{K}^{+}\right)$reaction predominantly matches to states of higher spin, implying the feasibility of populating "stretched" hypernuclear configurations. The $\left(\pi^{+}, \mathrm{K}^{+}\right)$reaction is therefore an important new spectroscopic tool, complementary to the $\left(\mathrm{K}^{-}, \pi^{-}\right)$ reaction, offering the possibility of extending detailed hypernuclear studies to heavier nuclei, bejond the p-shell. 
APPENDIX 3.3.3.a

The following is the TRANSPORT input file for the LESB-I beam line.

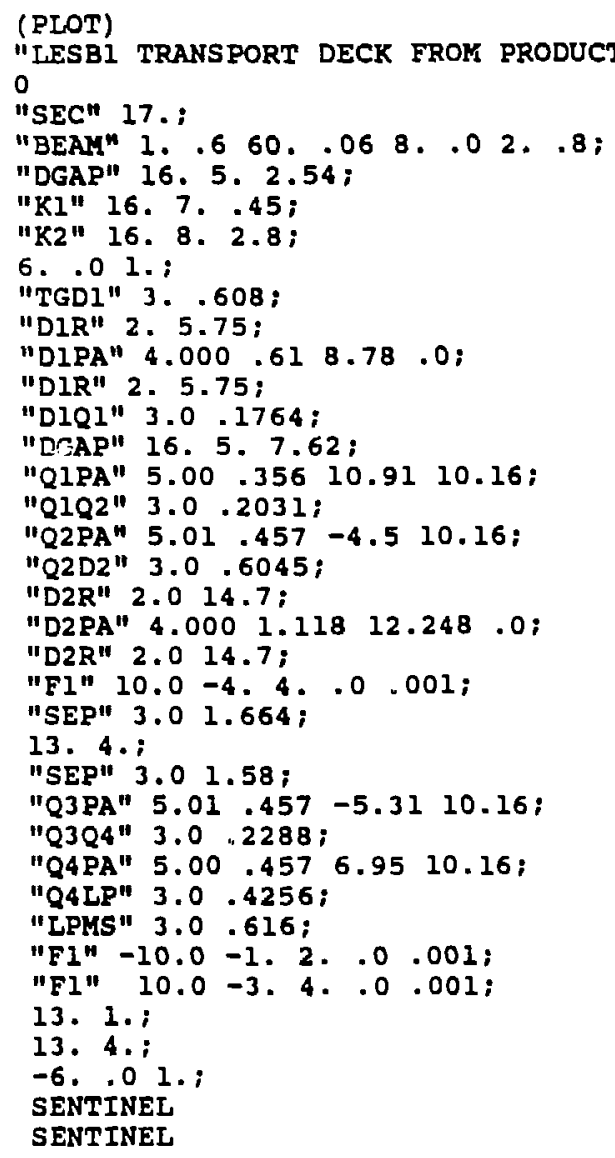


APPENDIX 3.3.4.a

Shown here are the data collected in the floating-wire m.: :ssurements for $S \bar{p} 2$.

DATA FOR SP2 D4 FROH THE FLOATING WIRE MEASUREMENT

$1.06819, .0005,1097.8, .1$

$.42871, .0005,500.9, .1$

$.61733, .0005,700.2, .1$

$.82305, .0005,897.3, .1$

$.72000, .0005,801.6, .1$

$.52050, .0005,599.6, .1$

$1.05247, .0005,1084.5, .1$

$.94453, .0005,998.8, .1$

$1.05237, .0005,1084.3, .1$

$.42456, .0005,496.3, .1$

$.67364, .0005,756.9, .1$

$.7608, .0005,840.4, .1$

$1.05523, .0005,1086.1, .1$

$.68708, .0005,770.6, .1$

$.68721, .0005,771.5, .1$

$.47708, .0005,554.2, .1$

$.56755, .0005,650.2, .1$

$.87643, .0005,944.8, .1$

$1.01503, .0005,1052.4, .1$

$.42908, .0005,501.6, .1$

$.61754, .0005,700.7, .1$

$.72200, .0005,804.1, .1$

$.82557, .0005,900.2, .1$

$1.06827, .0005,1097.2, .1$

D4: MOMENTUM, HALL; FROM JUN-83 WIRE DATA.

$506.18,0.1, .43200, .0005$

$556.34,0.1, .47815, .0005$

$605.86,0.1, .52484, .0005$

$655.84,0.1, .57240, .0005$

$701.70,0.1, .61670, .0005$

$718.50,0.1, .63320, .0005$

$752.03,0.1, .66655, .0005$

$801.93,0.1, .71794, .0005$

$852.74,0.1, .77132, .0005$

$903.47,0.1, .82665, .0005$

$953.67,0.1, .88434, .0005$

$1002.08,0.1, .94456, .0005$ 
Q9: MOMENTUM, HALL VOLTS

$541.5,0.1, .17188, .0005$

$632.9,0.1, .20317, .0005$

$720.2,0.1, .23439, .0005$

$819.3,0.1, .27171, .0005$

$998.5,0.1, .34360, .0005$

Q10: MOMENTUK, HALL VOLTS

$844.4,0.1, .17875, .0005$

$760.6,0.1, .16040, .0005$

$676.6,0.1, .14217, .0005$

$592.5,0.1, .12405, .0005$

\footnotetext{
CALIBRATION OF Q11 HALC VOLATAGE VS MOMENTUK $.3327, .0091,700,1$. $.3845, .0001,799.4,1$. $.4375, .0001,901.1,1$.

$.4879, .0001,997.7,1$.

$.5282, .0001,1078 ., 1$.
} 


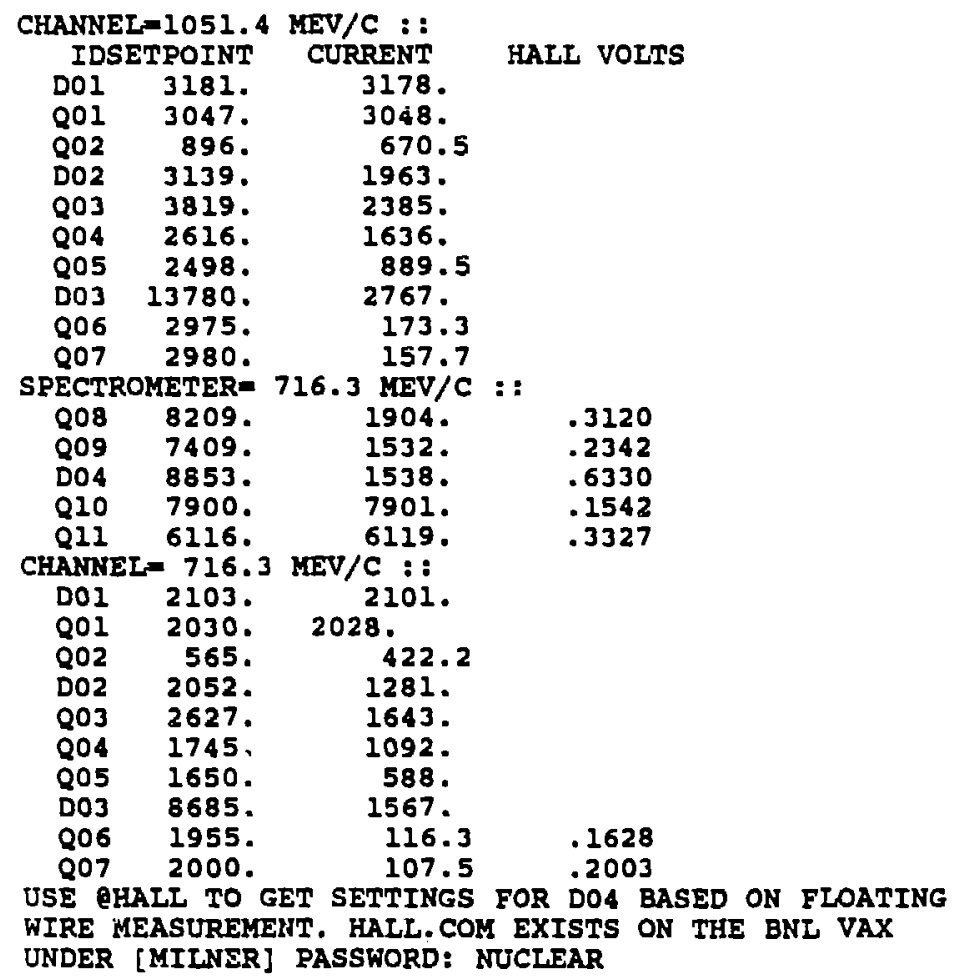


APPENDIX $3 \cdot 3 \cdot 4 \cdot \mathrm{b}$

The following is the TRANSPORT file for Sp1, along with a completed calculation.

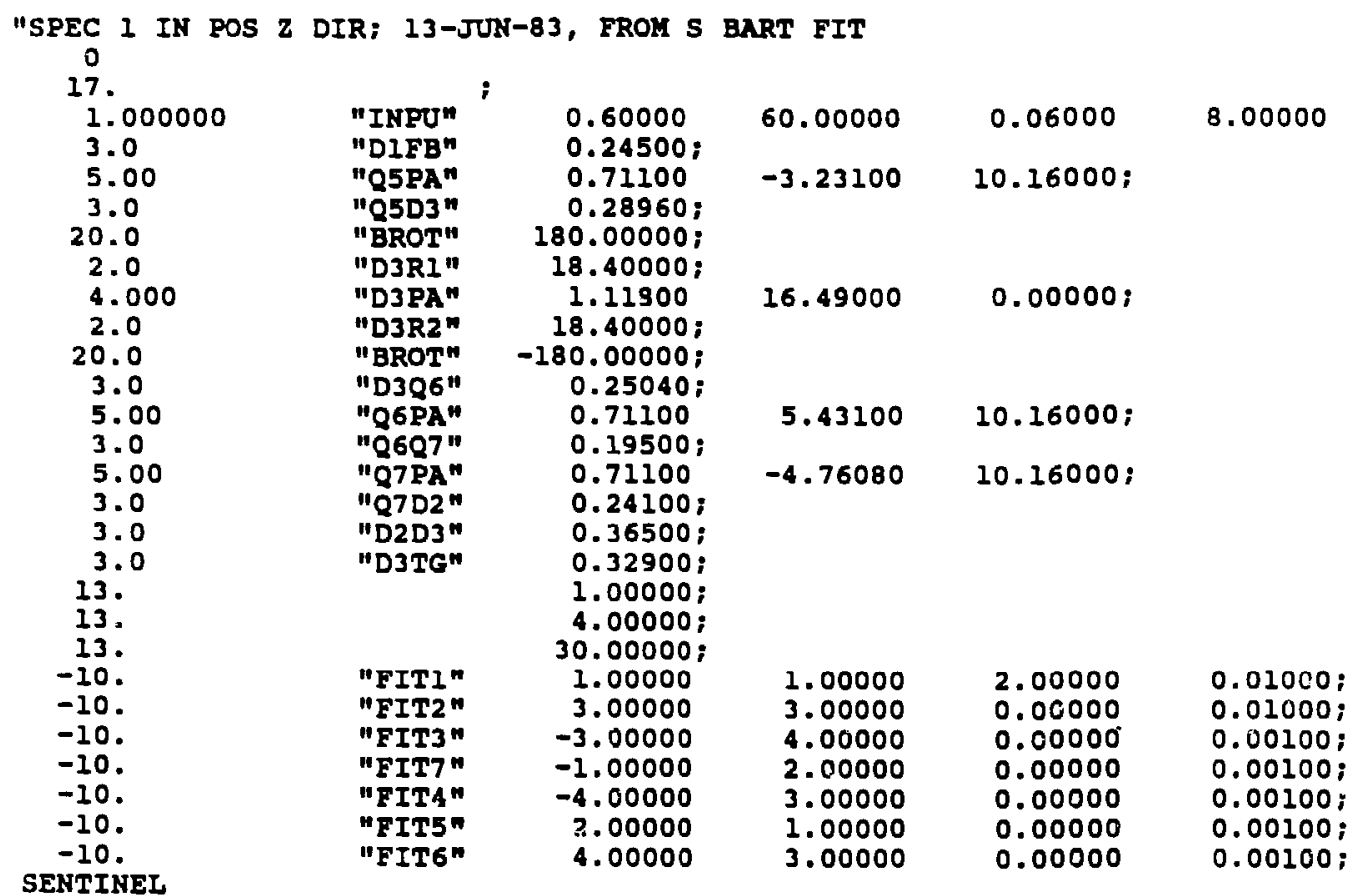


SPEC 1 IN FOS 2 DIR, 13-JUN-83, FROH I BART FIT

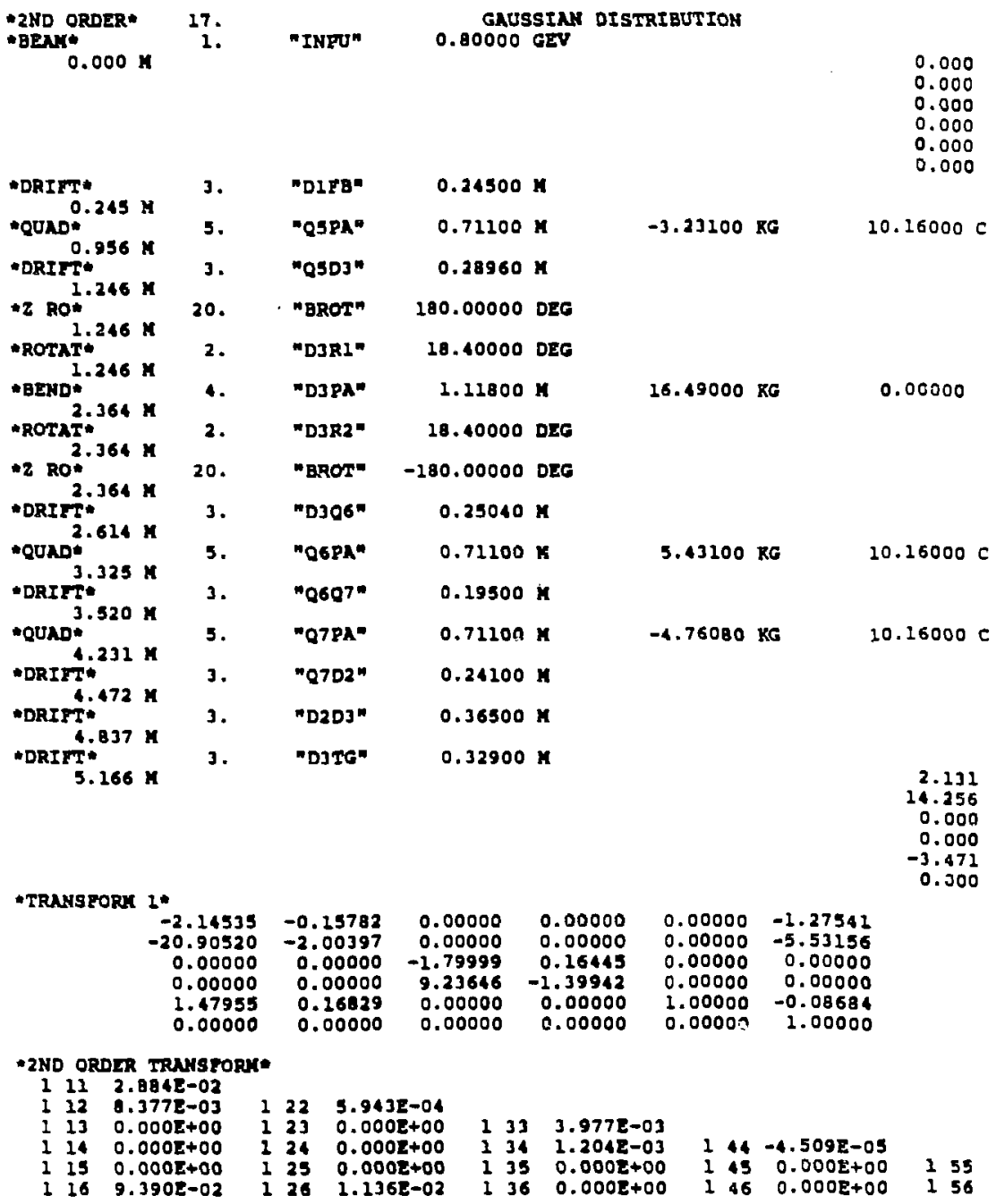




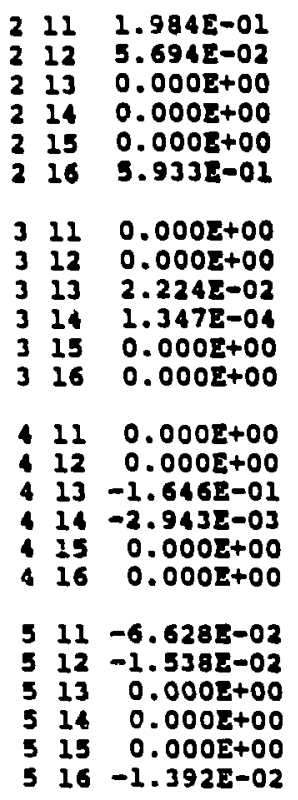

$2223.9735-03$

$2230.0005+00$

$2240.000 z+00$

$2250.000 \mathrm{E}+00$

226 6.658E-02

$3220.0002+00$

$323 \quad 3.256 \mathrm{E}-03$

$324 \quad 2.652 \mathrm{E}-05$

$3250.000 \mathrm{E}+00$

$3260.000 \mathrm{E}+00$

$4220.000 E+00$

$423-2.248 z-02$

$424-6.1352-04$

$4250.0008+00$

$+260.0005+00$

$522-9.485 E-04$

$5230.000 \mathrm{E}+00$

$5240.0002+00$

$5250.000 \mathrm{E}+00$

$526-5.179 \mathrm{E}-04$
$233 \quad 1.7572-02$

$234 \quad 9.841 E-03$

$235 \quad 0.0005+00$

$2360.0008+00$

$3330.000 z+00$

$3340.000 E+00$

3 $350.000 E+00$

$336-4.349 E-03$

344

345

346

$0.000 E+00$

$0.0005+00$

4.922E-03

355

356

$4330.000 E+00$

$4340.000 E+00$

$4350.000 \Sigma+00$

$436-3.938 E-0 I$

$4440.000 E+00$

$4450.000 E+00$

446 I.410E-02

455

456

$533-2.918 \mathrm{E}-02$

534 4.977E-03

$5350.0005+00$

$5360.000 \mathrm{E}+\mathrm{CO}$
$544-3.230 E-04$

$5450.000 \mathrm{E}+00$

$5460.000 E+00$
255

256

\section{- LENGTH*}

$5.16600 \mathrm{H}$ 


$\begin{array}{crcc}\text { DISTANCE } & \text { LABEL } & \mathrm{X} & \mathrm{Y} \\ 0.000 & \text { "INPU" } & 0.6000 & 0.0600 \\ 0.245 & \text { "DIFB" } & 1.5877 & 0.2050 \\ 0.956 & \text { "Q5PA" } & 6.6897 & 0.6547 \\ 1.246 & \text { "Q5D3" } & 9.3773 & 0.7757 \\ 1.246 & \text { "DJRI" } & 9.3774 & 0.7758 \\ 2.364 & \text { "DJPA" } & 18.8269 & 1.0685 \\ 2.364 & \text { "D3R2" } & 18.8356 & 1.0690 \\ 2.614 & \text { "D3Q6" } & 21.0491 & 1.0798 \\ 3.325 & \text { "Q6PA" } & 16.5623 & 1.7109 \\ 3.520 & \text { "Q6Q7" } & 12.5933 & 2.0773 \\ 4.231 & \text { "Q7PA" } & 2.6715 & 2.3689 \\ 4.472 & \text { "Q7D2" } & 2.8162 & 2.0990 \\ 4.837 & \text { "D2D3" } & 6.5411 & 1.6904 \\ 5.166 & \text { "D3TG" } & 10.4375 & 1.3225\end{array}$


APPENDIX 3.3.5.a

The following is the TRANSPORT input file for $\mathrm{Sp} 2$, along with a completed calculation.

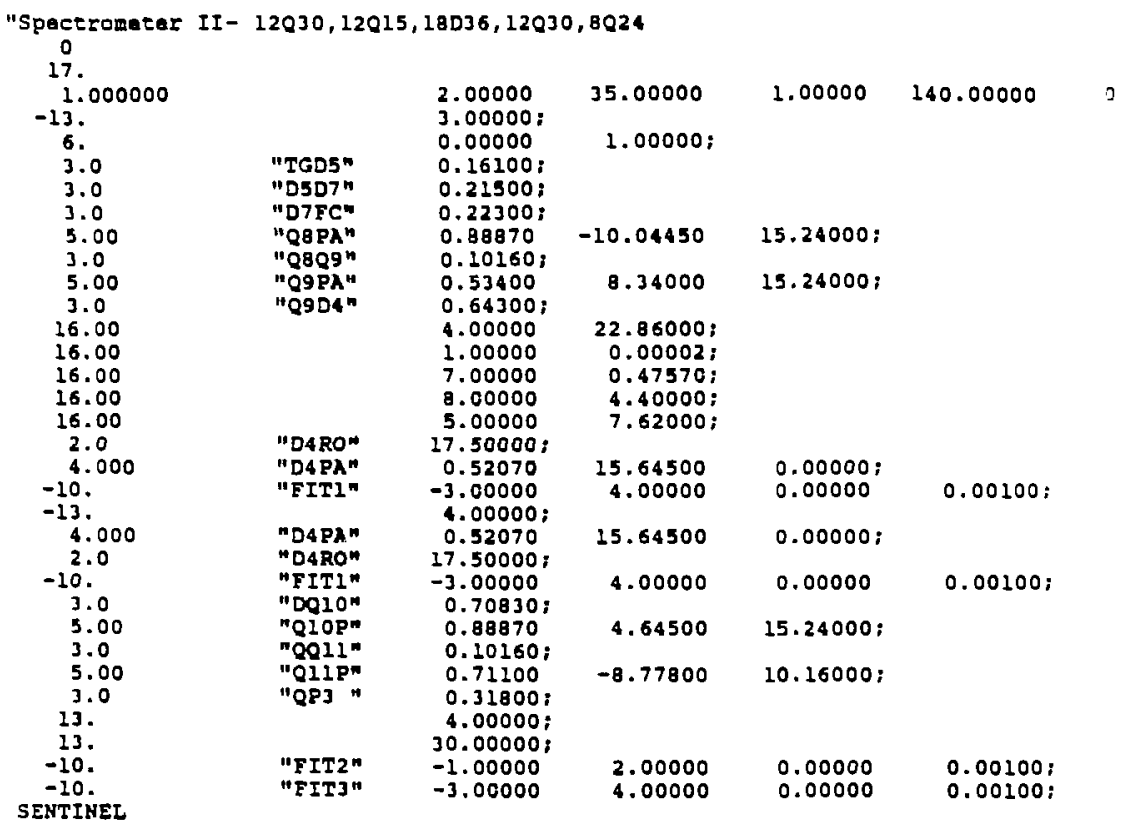


Spactrometer II- $12030,12015,18036,12030,8024$

-2ND ORDER 17.

ESNA*

1.

GAUSSIAK DISTRIBUTION

$0.000 \mathrm{H}$

$0.80000 \mathrm{GEV}$

0.000

0.000

0.000

0.000

0.000

0.000

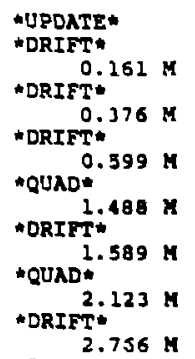

- PARAY"

-BETA

- KI.

K2

G/2"

$2.766 \mathrm{M}$

- BEND"

$3.287 \mathrm{~K}$

$3.808 \mathrm{H}$

ROTAT.

$3.808 H$

-DRIFT.

LUAD+
5.405

$5.405 \mathrm{~K}$

DRTFT"
$5.506 \mathrm{~K}$

-OUADE

$6.217 \mathrm{~K}$

-DRIFT"

$6.535 \mathrm{H}$

6. 10.$) 1$.

$0.16100 \mathrm{M}$

1.0

3. "D5D7" $0.21500 \mathrm{~K}$

3. "DTEC"

$0.22300 \mathrm{~K}$

5. "QgPA"

$0.88870 \mathrm{r}$

$-10.04450 \mathrm{kG}$

$15.24000 \mathrm{C}$

3. "Qgeg"

$0.10160 \mathrm{x}$

5. "Q9PA"

$0.53400 \mathrm{r}$

$8.34000 \mathrm{KG}$

$15.24000 \mathrm{C}$

3. "Q904"

$0.64300 \mathrm{x}$

16.

16.

16.

16.

16. "DARON 2.50000 DEG

4. $0.22860 \mathrm{E}+02$

1. $\quad 0.16000 \mathrm{E}=04$

7. $0.47570 \mathrm{E}+00$

8. $0.44000 \mathrm{E}+01$

5. $0.74000 \mathrm{E}+01$

4. "D4PA"

$0.52070 \mathrm{~K}$

$15.64300 \mathrm{KG}$

0.00000

4. "DAPA"

$0.52070 \mathrm{H}$

$\$ 5.64500 \mathrm{KG}$

0.00000

2. "DARO"

17.50000 TEE

3. "DQ10"

0.708394

5. "Q10P"

c.88870 $\mathrm{H}$

$4.64500 \mathrm{KG}$

15.24000

3. $\mathrm{NQQ11}$

$0.10160 \mathrm{~K}$

5. "Q11P"

$0.71100 \mathrm{H}$

$-8.77800 \mathrm{KG}$

$10.16000 \mathrm{C}$

TRANSFORY i.

3. HOPJ

$0.31800 \mathrm{M}$

$\begin{array}{rrrr}-0.66973 & 0.02481 & 0.00000 & 0.00000 \\ -15.21447 & -0.92946 & 0.00000 & 0.00000 \\ 0.00000 & 0.00000 & -2.11665 & -0.01974 \\ 0.00000 & 0.00000 & 130.09334 & 0.74110 \\ -1.82535 & -0.24910 & 0.00000 & 0.00000 \\ 0.00000 & 0.00000 & 0.00000 & 0.00000\end{array}$

0.00000

0.00000

0.00000

0.00000

1.00000

0.00000

2.12124

20.93383

0.00000

0.00000

$-0.06351$

-2ND ORDER TRANSFORH

$111-3.163 E-02$

$112-8.369 E-03$

$122-5.661 \mathrm{E}-0$

$1130.000 \mathrm{E}+00$

$1230.000 \mathrm{E}+00$

$133 \quad 1.916 E-03$ 


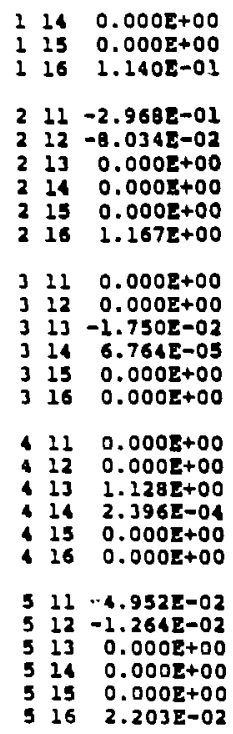

$\begin{array}{lll}1 & 24 & 0.000 E+00 \\ 1 & 25 & 0.0008+00\end{array}$

$125 \quad 0.000 E+00$

$134-1.0045-03$

$1360.000 E+00$

$222-5.5645-03$

$2230.0002+00$

$2240.0005+00$

$3250.000 z+00$

$2261.5075-01$

$3220.000 \mathrm{E}+00$

$323-2.645 E-03$

$3242.698 E-06$

$3250.0005+00$

$3260.000 E+00$

$334 \quad 0.0008+00$

3 3s $0.000 \mathrm{E}+00$

$4220.000 z+00$

423 1.575E-01

$4243.7732-04$

- $250.0005+00$

$4260.0002+00$

$4330.0002+00$

$4340.000 E+00$

$4350.0005+00$

$436=2.613 \mathrm{E}+00$

$522-8.911 E=04$

$5230.000 E+00$

$5240.0002+00$

$5250.000 E+00$

$526 \quad 7.771 E=04$

$5350.000 E+00$

$5360.000 E+00$
$1350.000 E+00$

3 $36-7.391 \mathrm{E}-02$

$533-5.931 E-01$

$534-7.870 \mathrm{E}-03$

$144-3.822 E-06$

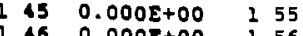

$1460.00025+00 \quad 156$

$331.301 \mathrm{E}-01$

35 $0.0005+00$

$244-4.6178-05$

$2450.000 \mathrm{E}+00$

$2460.000 E+00$

255

256

$344 \quad 0.0005+00$

$345 \quad 0.000 E+00355$

3461.619 E-03 3 56

$440.000 E+00$

$4450.000 E+00$

$446-1.498 E-01$

455 456

$544-8.221 E-05$

$545 \quad 0.000 E+00 \quad 555$ $546 \quad 0.000 E+00 \quad 556$ 


\begin{tabular}{|c|c|c|c|}
\hline ISTAKCE & EABEL & $\mathbf{x}$ & $\mathbf{Y}$ \\
\hline $\begin{array}{l}0.000 \\
0.161 \\
0.376 \\
0.599 \\
1.488 \\
1.589 \\
2.123 \\
2.766 \\
2.766 \\
2.766 \\
2.766 \\
2.766 \\
2.766 \\
2.766 \\
3.287 \\
3.808 \\
3.808 \\
4.516 \\
5.405 \\
5.506 \\
6.217 \\
6.535\end{array}$ & 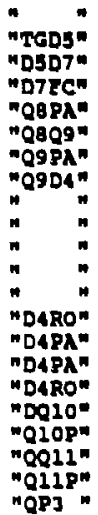 & $\begin{array}{r}2.0000 \\
2.0779 \\
2.3941 \\
2.8975 \\
9.7217 \\
11.2450 \\
15.3616 \\
15.1502 \\
15.1502 \\
15.1502 \\
15.1502 \\
15.1502 \\
15.1502 \\
15.1508 \\
15.7306 \\
14.9405 \\
14.9394 \\
15.1517 \\
9.5760 \\
8.3873 \\
7.8470 \\
11.1986\end{array}$ & $\begin{array}{r}1.0000 \\
2.4659 \\
5.3581 \\
8.4454 \\
10.2380 \\
9.1703 \\
5.9359 \\
4.9648 \\
4.9648 \\
4.9648 \\
4.9648 \\
4.9648 \\
4.9648 \\
4.9649 \\
4.5711 \\
5.0510 \\
5.0498 \\
6.2124 \\
11.9708\end{array}$ \\
\hline
\end{tabular}


APPENDIX 3.5.2.2.a

The following is the $Q$ data acquisition system test file used in data collection and replay.

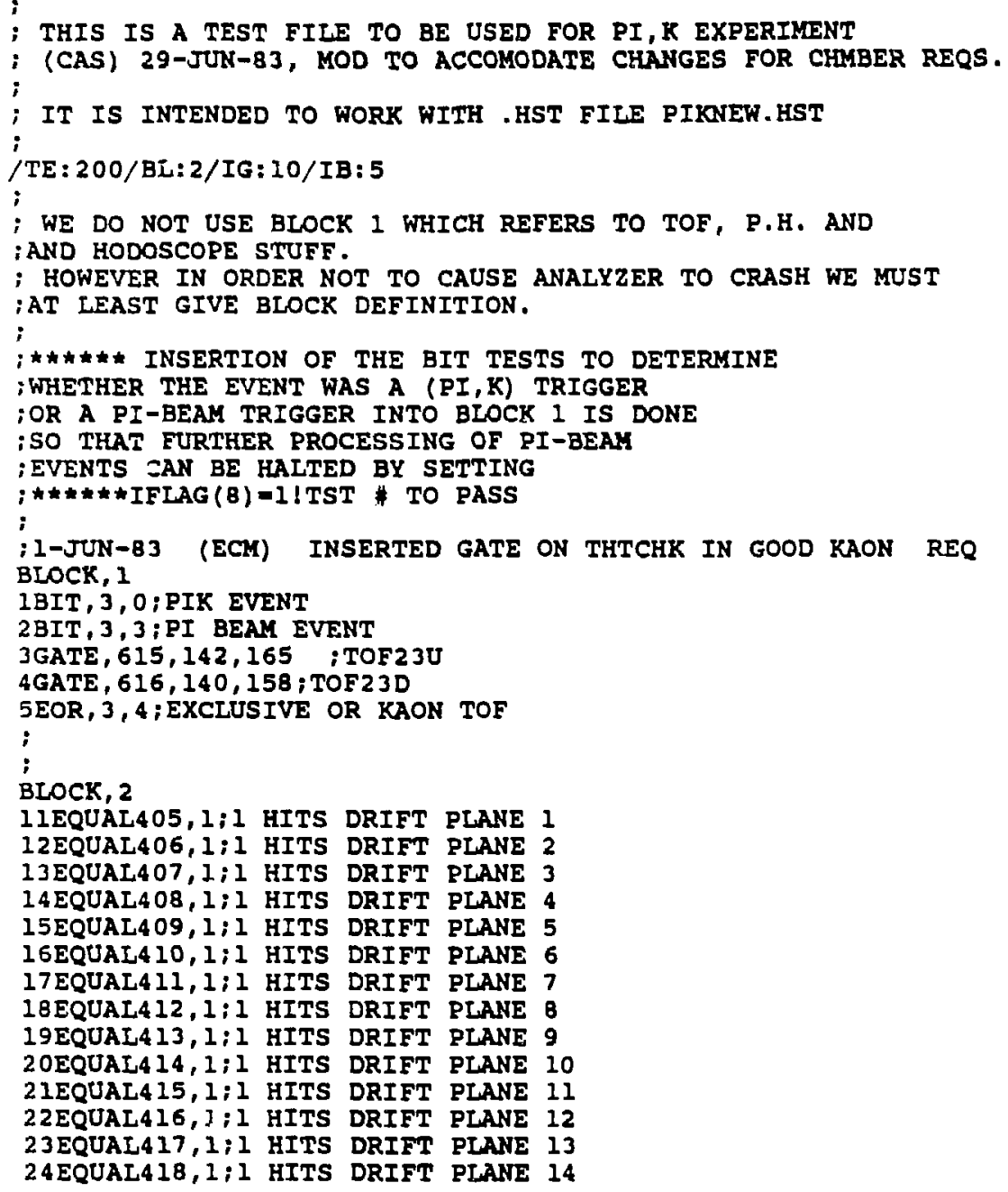




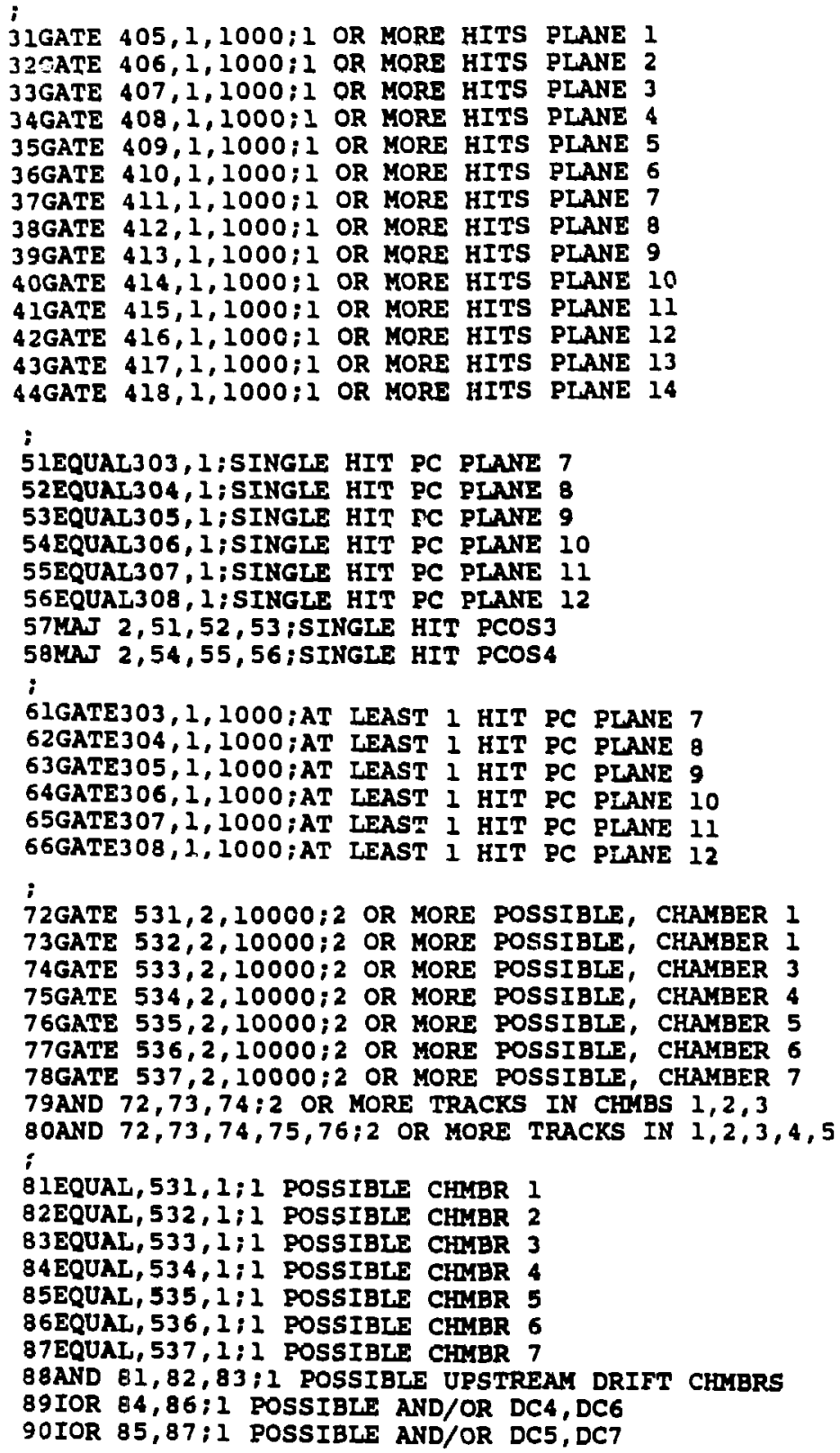


91EQUAL, 531, 0:0 POSSIBLE CHMBR 1

92EQCAL, 532,0;0 POSSIBLE CHMBR 2

93EQUAL, 533,0;0 POSSIBLE CHRBR 3

94EQUAL, 534,0;0 POSSIBLE CHLBR 4

95EQUAL, 535, 0;0 POSSIBLE CHMBR 5

96EQUAL, 536,0;0 POSSIBLE CHMBR 6

97EQUAL, 537, 0;0 POSSIBLE CHMBR 7

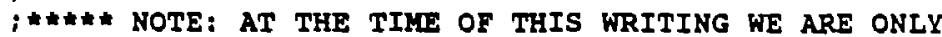
;USING DRIFTS $1,2,3,5,7$ AND PCOS $7,8,10,11 \hbar^{*} \hbar_{\hbar}$

101AND 88,90:1 POSS'BL DRIFT CHABRS $2,2,3,(5.7)$

102AND 57,58; EXACTLY 1 HIT, CMU 7\&8, 10,11

103AND 101,102;'GOOD EVENT' CHAMBERS

;

104IBOX 1;BOX I SET ON ZCLOSE.VS.XTGDIF

105GATE $727,-270,270$; SOLID ANG IN THT VS. $X$

106GATE $729,-800,800$; SOLID ANG IN PHI VS. $Y$

107IGATE, 9 ;GATE, 9 , PID ON STGT+S3U

IOSIGATE, 8 ;GATE 8 , GATE ON RXTGTU

109IGATE, 1;G 1 TE 1, SET ON PLSHT LIVE TGT

11OIGATE, 2 ;GATE 2 , SET ON KAONS IN TS23U

\author{
IIIIGATE, 3;GATE 3 , SET ON KAONS IN TS23D \\ 112 IGATE, 4;GATE 4 , SET ON THCHNR \\ 113IGATE, 5;GATE 5 ; SET ON YSPCR \\ 114IGATE, 6;GATE 6; DEFINES PHI FOR SOLID ANG \\ ll5IGATE, 7 ; GATE 7 , SET ON THTCHR
}


;

116EOR 110,111;EXCLUSIVE OR ON RAON TOF

117AND 103,104,112,113,115;GOOD EVENT- CHM\#BOX1*THCHNR*YSPCR*THCK

118AND 116,117;GOOD TGT EVT * KAON TOF

119AND 118,114; GOOD EVENT TOF1?

120AND 109,118; >>> GOOD KAON<<<<

121AND 103,104iSIMPLE EVENT TEST=CHMBRS * TGT BOX

122AND 88,112; (UPSTR CABRS THCHNR)=GOOD BEAK

123AND 122,$108 ;$ GOOD BEAH * XTGT

124 AND 118,-109;GOOD KAON * NOT PLSHT

125AND 118,105,106;GOOD KAON * SOLID ANGLE

126AND 125,108;KAON SOLID * XTGTU

127AND 118,108;KAON * XTGTU

128AND 103,$116 ;$ CHABRS TOF

129AND 121,116;CHMBRS * BOX1 * TOF

130AND 88,89,90;ALL CHAKBERS

131 AND $11,12,13,14,15,16$;ONE HIT ONLY IN UPSTREAM

132 AND $131,116,102$;UPसTOF*CAN

133 AND $132,-85$;UP*TOF*CNU*-DC5

134 AND $132,-87$;UP*TOF $\star C A U \star-D C 7$

135AND 90,102,116; PID*DOWNSTREAK CHAMBERS

136 AND 135,131 ;PID*IOR DOWN*UP ONE HIT

137 AND 133,134 ; NEITHER DC5 NOR DC7 FIRED WHEN ONE SHOULD

138 AND 102,116 :TEST TO GET CMU EFF

139 AND 131,116 ;UP*TOP

140 AND $131,116,90 ;$;UP*TOF $\star$ IOR 57

; SOLID AKGLE BOX

141 IBOX 2

;SET ON THSPCF VS RXTGTU

142 AND $118,106,141 ; K \times P H I * T H T * X$

143 EQUAL 303,0 ;NO HIT PCP7

144 EQUAL 304,0; NO HI' PCP8

145 EQUAL 305,0 ;NO HIT PCE9

146 EQUAL 306,0 iNO HIT PCP10

147 EQUAL 307,0 ;NO HIT PCPII

148 EQUAL 308,0 ;NO HIT PCP 12

149 MAJ $1,143,144,145$;ONE ZERO HIT IN PC3

150 MAJ 1,146,147,148 ;ONE ZERO HIT IN PC4

151 MAJ 2, 143,144,145 ; TWO 2ERO HITS IN PC3

152 MAJ $2,146,147,148$;TWO ZERO HITS IN PC4

153 MAJ $3,143,144,145$; TRREE 2ERO HITS IN PC3

154 MAJ $3,146,147,148$; THREE ZERO HITS IN PC4

;TWO HIT TESTING:

155 EQUAL 303,2

156 EQUAL 304,2

157 EQUAL 305,2

158 EQUAL 306,2

159 EQUAL 307,2

160 EQUAL 308,2

12 HITS PCP7

12 HITS PCPB

; 2 HITS PCP9

;2 HITS PCP 10

;2 HITS PCPI1

; 2 HITS PCP12

161 MAJ 1, 155, 156, 157 ;SING 2 HIT IN PCJ

$162 \mathrm{MAJ} 1,158,159,160$

163 MAJ $2,155,156,157$

;SING 2 HIT IN PC4

$164 \mathrm{MAJ} 2,158,159,160$ ; TWO 2 HITS IN PC3

165 MAJ $3,155,156,157$ ;THO 2 HITS IN PC4

166 MAJ $3,158,159,160$

i 3 HITS IN PC3

i 3 HITS IN PC4 


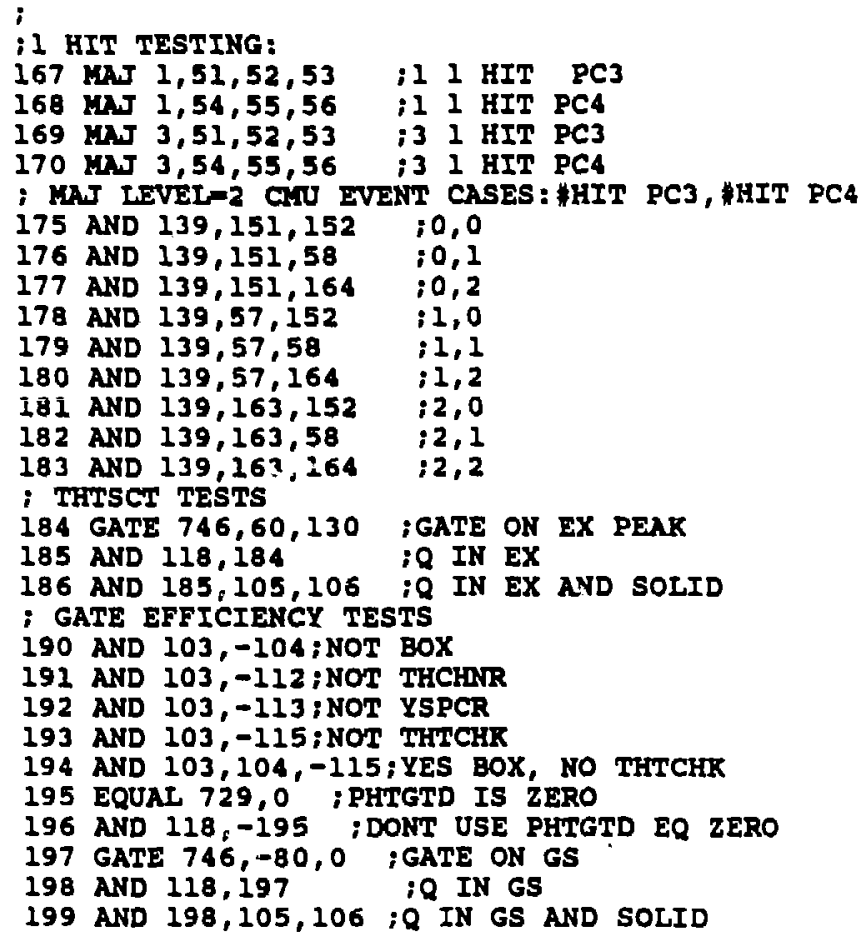


APPENDIX 4.1.a

The following table displays the unshifted ${ }^{12} \mathrm{C}\left(\pi^{+}, \mathrm{K}^{+}\right){ }_{\Lambda}^{12} \mathrm{C}$ spectra data from the histogram QSOLID. These events passed all good event requirements, and fell into the software restricted solid angle region. Counts appearing after the $1 / \|$ symbol represent the estimated background for that bin.

Spectrometer Angle

$\begin{array}{cccc}\text { Eex }^{(\mathrm{MeV})} & 5.6^{\circ} & 10.3^{\circ} & 15.2^{\circ} \\ -20 & 1 & 3 & 0 \\ -19 & 0 & 1 & 0 \\ -18 & 1 & 6 & 1 \\ -17 & 0 & 7 & 0 \\ -16 & 2 & 7 & 0 \\ -15 & 0 & 3 & 4 \\ -14 & 3 & 9 & 0 \\ -13 & 0 & 8 & 2 \\ -12 & 3 & 6 & 1 \\ -11 & 0 & 9 & 3 \\ -10 & 1 & 9 & 0 \\ -9 & 2 & 15 & 1 / 1 \\ -8 & 0 / 1 & 7 & 3 / 1 \\ -7 & 1 / 1 & 12 / 10 & 4 / 1 \\ -6 & 3 / 1 & 29 / 10 & 3 / 1 \\ -5 & 11 / 2 & 52 / 10 & 5 / 1 \\ -4 & 15 / 2 & 49 / 11 & 6 / 1 \\ -3 & 24 / 2 & 43 / 11 & 3 / 1 \\ -2 & 8 / 2 & 25 / 11 & 4 / 1 \\ -1 & 7 / 3 & 21 / 12 & 1 / 1 \\ 0 & 3 / 3 & 12 / 12 & 0 \\ & & & \end{array}$




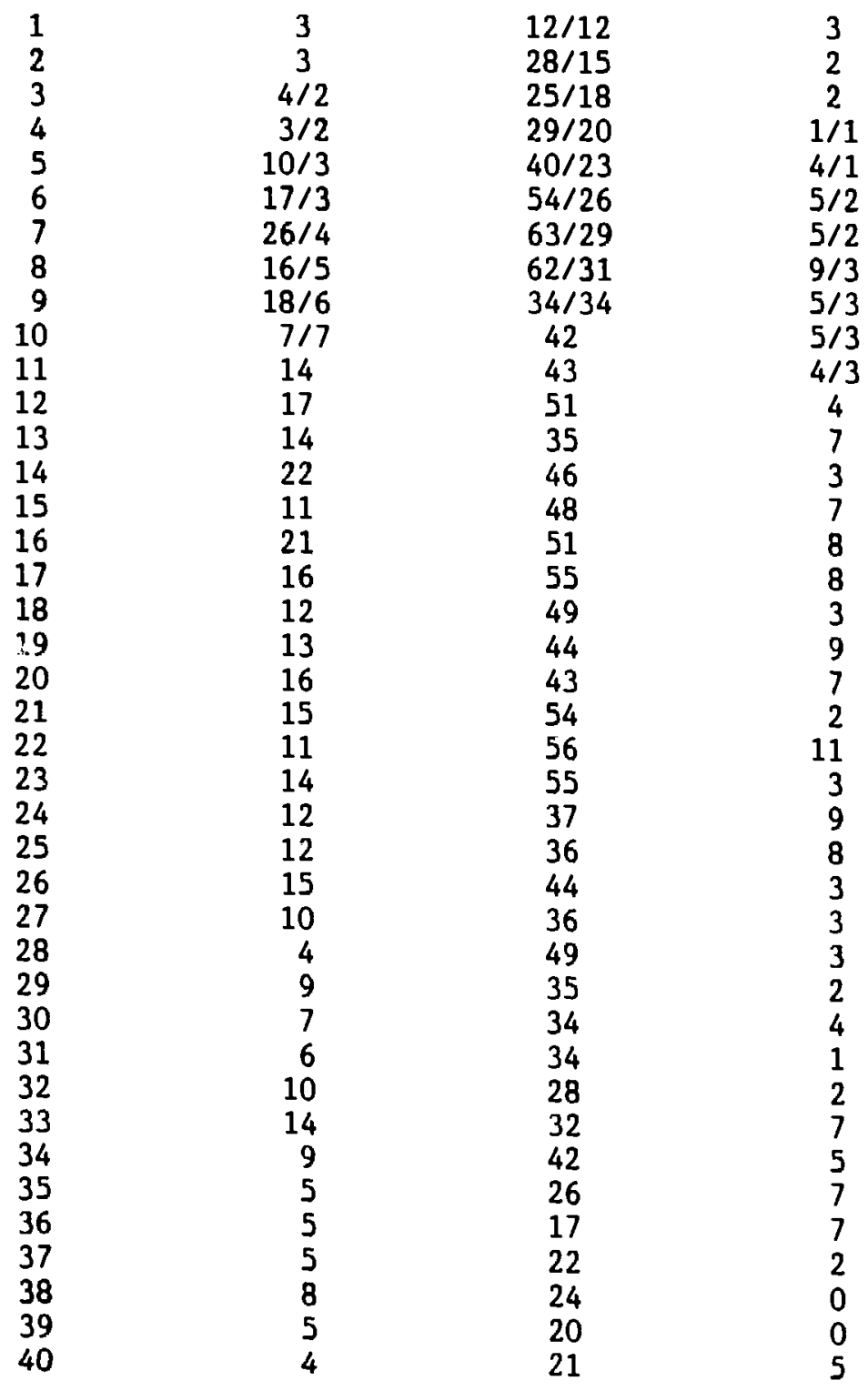


APPENDIX 5.2.a

The following are the three CHUCK input files used to calculate the $\mathrm{J}^{\pi}=2^{+}, 1^{-}$, ard $0^{+}$contributions to the ${ }^{12} \mathrm{C}\left(\pi^{+}, \mathrm{K}^{+}\right) \mathrm{S}^{12} \mathrm{C}$ differential scattering cross sections.

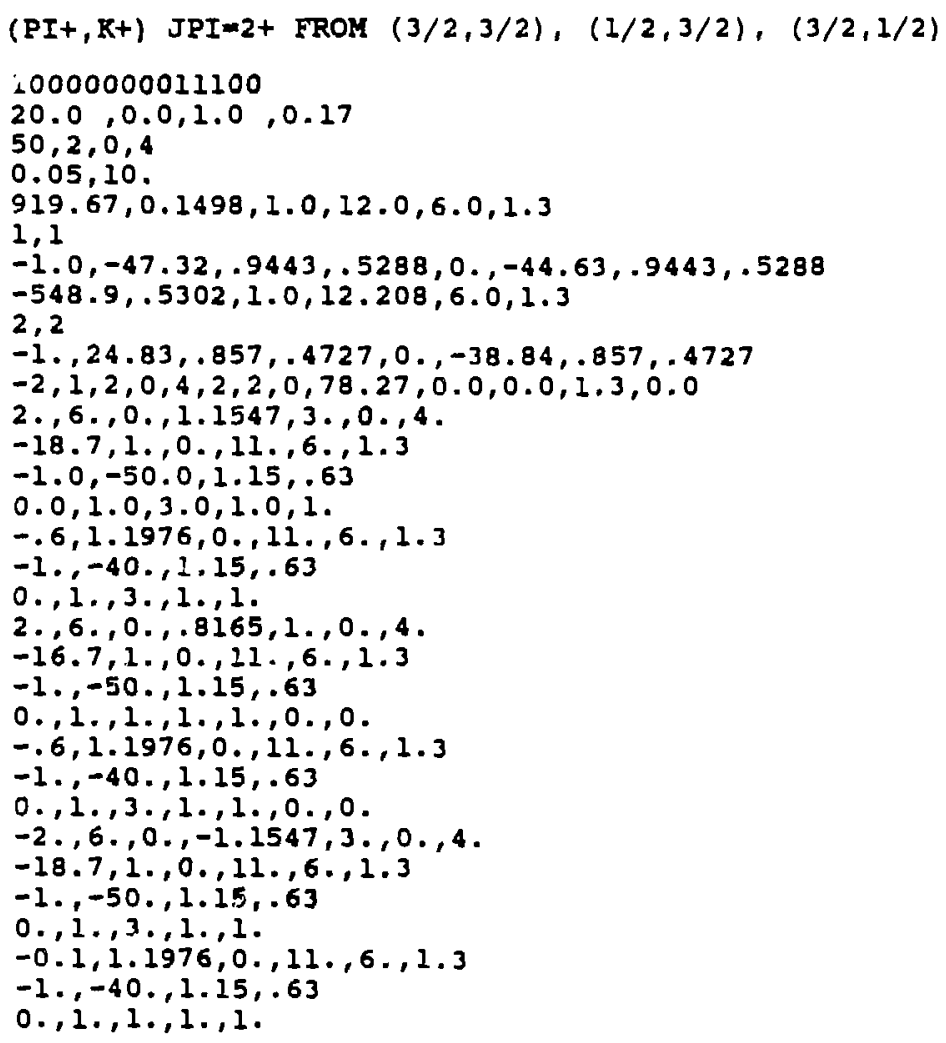




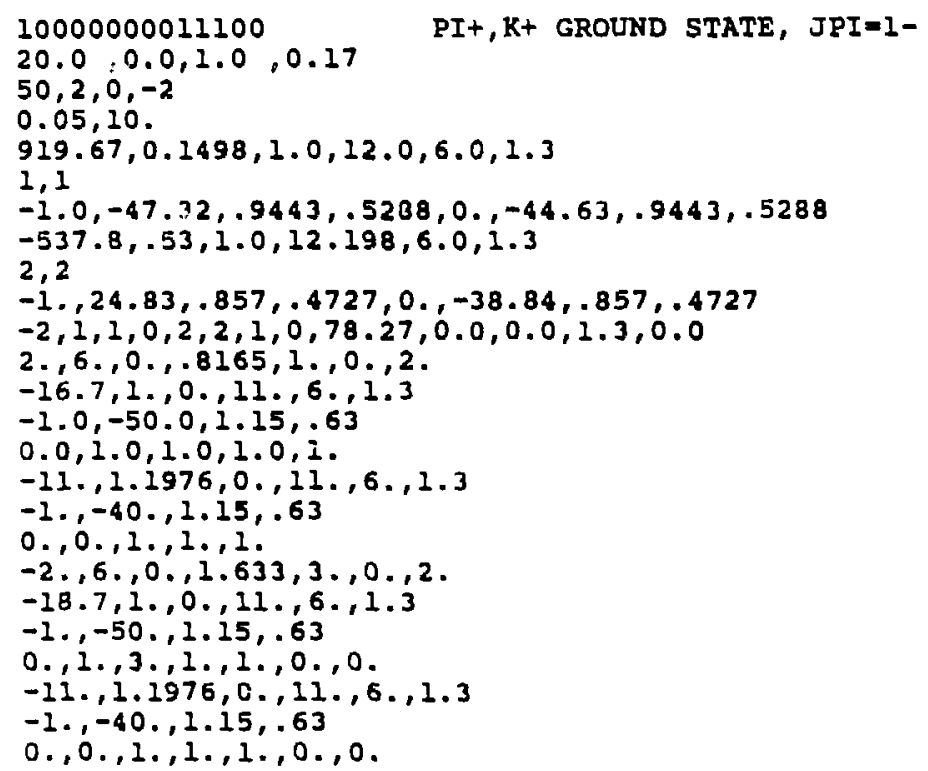




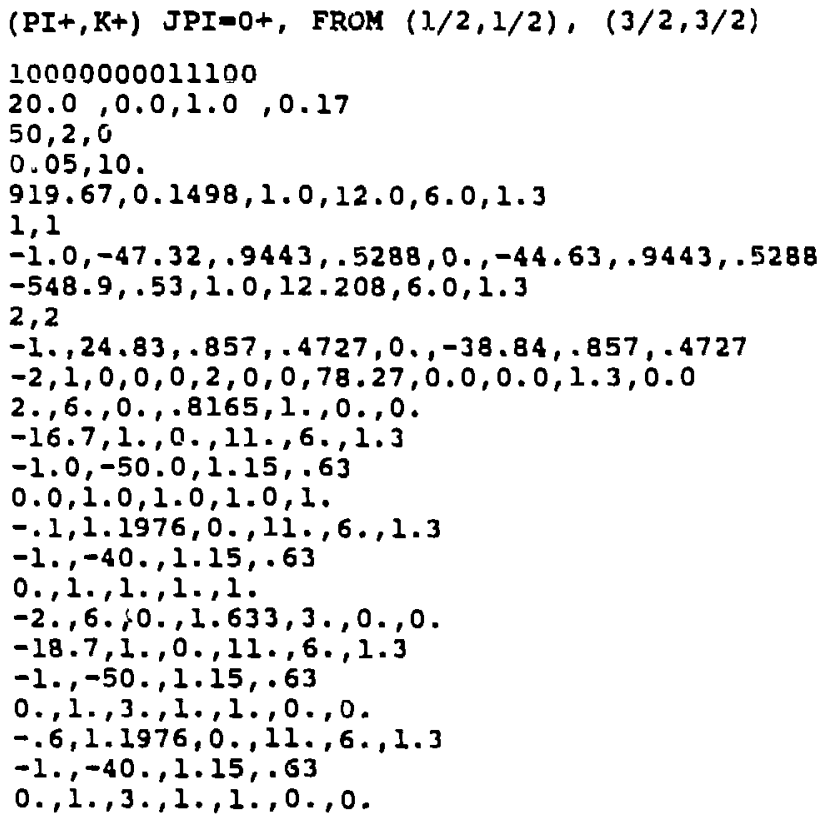




\section{REFERENCES}

[Am 82] J. F. Amann et al., LANL Report LA9486-MS, (1982).

[Au 83] E. B. Auerbach, A. J. Baltz, C. B. Dover, A. Gal, S. H. Kahana, L. Ludeking, and D. J. Millener, Ann. of Phys. 148, 381 (1983).

[Be 81] A. H. Bernstein, T. W. Donnelly, and G. N. Epstein, Nucl. Phys. A358, 1956 (1981).

[Bo 70] G. Bohm et al., Nucl. Phys. B24, 246 (1970).

[Bo 75] G. C. Bonazzola et al., Phys. Rev. Lett. 34, 683 (1975).

[Br 69] K. L. Brown, SLAC-75, (1969).

[Br 75] W. Brückner et al., Phys. Lett. 55B, 107 (1975).

[Br 77] K. L. Brown et al., "Transport", SLAC-91, (1977).

[Ch 79] R. E. Chrien et al., Phys. Lett. 89B, 31 (1979).

[Ch 85] R. E. Chrien et al., "Proposal for a $2.0 \mathrm{GeV} / \mathrm{C}$ Hypernuclear Beam Line", unpublished (1985).

[Co 52] E. D. Courant, M. S. Livingston, and H. S. Snyder, Phys. Rev. 88, 1190 (1952).

[Da 58] R. B. Dalitz and B. H. Downs, Phys. Rev. 111, 967 (1958). 
[De 65] D. Dekkers et al., Phys. Rev. 137, B962 (1965).

[Do 79] C. B. Dover, A. Gal, G. E. Walker, and R. H. Dalitz, Phys. Let t. 89B, 26 (1979).

[Do 80] C. B. Dover, L. Ludeking, and G. E. Walker, Phys. Rev. C22, 2073 (1980).

[En 84] B. A. Enge and S. B. Kowalski, AIP Conf. Proc. No. 123, 824 (1984).

[Fa 81] Farr and Weiskat, Nucl. Instr. Meth. 190, 35 (1981).

[Pe 66] H. Feshbach and A. K. Kerman, Preludes in Theoretical Physics, Nor th-Holland Pub. Co., Amsterdam, 260 (1966).

[Gr 85] D. D. Grace et al., "Lifetime of $\Lambda$ in $\Lambda^{12} \mathrm{C}$ ", submitted to Phys. Rev. Lett. (1985).

[Ko] S. B. Kowalski and H. A. Enge, unpublished. See H. A. Enge, Nucl. Instr. Meth. 162,161 (1979), and references therein.

[Ku] P. D. Kunz, unpublished.

[Ma 82] D. Marlow et al., Phys. Rev. C25, 2619 (1982).

[Ma 84] D. Marlow et al., Phys. Rev. C30, 1662 (1984).

[Mo 84] 0. Morimatsu et al., Nucl. Phys A240, 573 (1984).

[Pi 79] H. J. Pirner, Phys. Lett. 85B, 190 (1979).

[Pi 84] P. H. Pile, AIP Conf. Proceedings No. 123, 814 (1984).

[Pn 72] J. Pniewski, Proc. Int. Conf. on Few-Particle Problems in Nuclear Interactions, Los Angeles (Amsterdam: North-Holland), 146 (1972). 
[Ro 70] D. H. Rote and A. R. Bodmer, Nucl. Phys. A148, 97 (1970). [Ya 85] T. Yamazaki et al., Phys. Rev. Lett. 54, 102 (1985). 